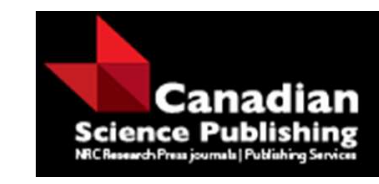

Canadian Journal of Physics

Revue canadienne de physique

\title{
COLLISION STRENGTHS AND EFFECTIVE COLLISION STRENGTHS \\ FOR Ne-LIKE Ge
}

\begin{tabular}{|r|l|}
\hline Journal: & Canadian Journal of Physics \\
\hline Manuscript ID & cjp-2016-0771.R3 \\
\hline Manuscript Type: & Article \\
\hline Date Submitted by the Author: & 28-Mar-2017 \\
\hline Complete List of Authors: & $\begin{array}{l}\text { Abdelaziz, Wessameldin S.; Cairo Univ, laser metrology; Laser Institute } \\
\text { 9NILES), }\end{array}$ \\
\hline Keyword: & $\begin{array}{l}\text { effective collision strength, laser, atomic, COLLISION STRENGTHS, Ne-LIKE } \\
\text { Ge }\end{array}$ \\
\hline $\begin{array}{r}\text { Is the invited manuscript for } \\
\text { consideration in a Special } \\
\text { Issue? : }\end{array}$ & N/A \\
\hline
\end{tabular}




\title{
COLLISION STRENGTHS AND EFFECTIVE COLLISION STRENGTHS FOR Ne-LIKE Ge
}

\author{
Wessameldin S.Abdelaziz \\ National institute of laser Enhanced Science, Cairo University, Giza, Egypt
}

\begin{abstract}
We have carried out a calculation of 241 levels for Ne-like Ge $(Z=36)$ in order to calculate electron impact collision strengths and effective collision strengths which belonging to $1 s^{2} 2 s^{2} 2 p^{5} n l, 1 s^{2} 2 s^{1} 2 p^{6} n l(n=3,4,5,6$; $\mathrm{l}=\mathrm{s}, \mathrm{p}, \mathrm{d}, \mathrm{f}, \mathrm{g}$ and $\mathrm{h}$ ) configurations which have been calculated by the fully relativistic flexible atomic code (FAC). Our calculations based on the distorted-wave method with large configuration interactions included. Collision strengths have been generated over an electron energy range of (10 --- $20000 \mathrm{eV}$ ) and they have been listed at seven representative energies of 65.33, 1714.98, 2944.1, 4868.3, 7564.6, 11040 and 15221eV in this work, and effective collision strengths data have been calculated from these at electron plasma temperatures $(650,850,1050,1250,1450,1650,1850) \mathrm{eV}$. Our results are compared with those available in the literature. It is found that the resonance effects are important in calculating the effective collision strengths.
\end{abstract}

* Corresponding author.

E-mail address: wessamlaser@yahoo.com 


\section{Contents}

1. Introduction

2. Theoretical method

3. Energy levels and oscillator strengths

4. Collision strengths and effective collision strengths

5. Conclusions

References

Explanation of Tables

\section{Introduction}

Electron impact collision strengths and effective collision strengths are of fundamental importance in analyzing the physical state and chemical composition of gaseous nebulae and laboratory plasmas. At present it is difficult to obtain such information experimentally; therefore, it has become necessary to rely on the results of quantum-mechanical calculations. The ions of germanium have along been important in astrophysical application but since their recent discovery in the plasma, the general interest in these ions has dramatically increased. Because Nelike ions have a stable closed L-shell ground state, they show high abundance over a wide range of temperatures in ionization equilibrium for each iso-nuclear sequence (see Mazzotta et al. 1998, Bryans et al. 2006; 2009) [1,2,3]. Thus, they attract extensive studies for spectral diagnostic and modeling in astrophysical and laboratory plasmas, and in particular iron, due to its high cosmic abundance. X-ray lasers (Mathews et al. 1985, Tomasel et al.1997) [4,5] based on Ne-like ions are another significant area of interest. However, the atomic structure and electron impact excitation (EIE) of Ne-like ions are extremely complex, which results in there being large uncertainties in line intensity ratios $\left(2 p^{5} 3 d^{1} \mathrm{P}_{1} \rightarrow 2 p^{61} \mathrm{~S}_{0}\right.$ vs $2 p^{5} 3 d^{3} \mathrm{D}_{1} \rightarrow$ $2 p^{6} S_{0}$, this is usually designated $3 C / 3 D$, as well as
$3 s-2 p$ vs $3 C$ ) between measurements or observations and predictions (Beiersdorfer et al. 2001; 2002, Gu et al. 2004) [6,7,8]. For example, even for iron, EIE of this ion has been investigated experimentally and theoretically for a long time (Smith et al. 1985, Chen et al. 2003, Loch et al. 2006, Beiersdorfer et al. 2001; 2002 and references therein) $[9,10,11,6,7]$. Resonances in electron-ion impact excitation have been observed in laboratory measurements (Brown etal. 2006) [12]. They play an important role in the spectral diagnostic and modeling of astrophysical and laboratory plasmas. On the other hand, collision strengths for electron impact excitation of Ne-like ions are also affected by the strong configuration mixing. Zhang and Sampson [13] systematically calculated the collision strengths for electron impact excitation to $n=3$ and 4 excited levels in Ne-like ions using the fully relativistic distortedwave method. Their calculation showed that the collision strengths for $\left(2 p_{1 / 2}^{-1} 3 S\right)_{J=1}$ and $\left(2 p_{3 / 2}^{-1} 3 d_{5 / 2}\right)_{J=1}$ have Z-independence similar to that of the oscillator strengths, i.e. the collision strengths for the $2 p^{6} \rightarrow\left(2 p_{1 / 2}^{-1} 3 S\right)_{J=1}$ excitation is enhanced while that for the $2 p^{6} \rightarrow$ $\left(2 p_{3 / 2}^{-1} 3 d_{5 / 2}\right)_{J=1}$ excitation is suppressed with $\mathrm{Z}$ $=22 \sim 55$. In the present work we give the collision strengths and effective collision strengths for the first ten fine-structure levels between the ground 
level and the $n=3,4,5$ and 6 levels of Ge XXIII. The data are compared with those given in Honglin Zhang and Douglas H. Sampson (1989) [14].

\section{Theoretical method}

The calculations of atomic structure and electron impact excitation have been carried out using the Flexible Atomic Code (FAC) developed by Gu [15]. A fully relativistic approach based on the Dirac equation is used throughout the entire package. In the calculation of atomic structure, the energy levels of an atomic ion are obtained by diagonalizing the relativistic Hamiltonian. An atomic state is approximated by a linear combination of configuration state functions (CSFs) with same symmetry

$\phi_{\alpha}(J \pi)=\sum_{i}^{n_{c}} a_{i}(\alpha) \boldsymbol{\varphi}_{\alpha}(J \pi)$

where, $n_{c}$ is the number of CSFs and, $a_{i}(\alpha)$ denotes the representation of the atomic state in this basis. The CSFs are anti-symmetrized products of a common set of orthonormal orbitals which are optimized on the basis of the relativistic Hamiltonian. The radial orbitals are derived from a modified Dirac-Fock-Slater iteration on a fictitious mean configuration with fractional occupation numbers, representing the average electron cloud of the configurations included in the calculation. Once the $\mathrm{Cl}$ wavefunctions have been obtained, the radiative transition rates can be calculated in the single multipole approximation with arbitrary rank. The electron impact excitation has been calculated using the relativistic distorted-wave approximation. Special attention has been paid to the long range contributions to the continuumcontinuum radial integral by using the phaseamplitude method for the continuum wavefunctions. To ensure the convergence of the collision strengths, large angular momentum contributions (the maximum partial waves up to 50) have been taken into account. Higher partial wave contributions have been included using the Coulomb-Bethe approximation [16]. Using the method described above, we calculated the energy levels, spontaneous radiative decay rates, oscillator strengths, and electron impact collision strengths for transitions among the 241 finestructure levels belonging to the $1 s^{2} 2 s^{2} 2 p^{5} n l, 1 s^{2}$
$2 s^{1} 2 p^{6} \mathrm{nl}(n=3,4,5,6 ; l=s, p, d, f, g$ and $h)$ configurations of Ne-like $\mathrm{Ge}$. $\mathrm{Cl}$ between the above configurations has been included in the calculations of atomic structure and collision strengths. For convenience of use, the effective collision strengths have also been obtained at electron plasma temperatures $(650,850,1050$, $1250,1450,1650,1850) \mathrm{eV}$.

\section{Energy levels and oscillator strengths}

In this section, we deal with the atomic structure and calculated energy levels and oscillator strengths [17]. The energy levels are calculated with the notation for J-J atomic states corresponding to configurations $1 \mathrm{~s} 2 \mathrm{2s} 2 \mathrm{2p} 5 \mathrm{nl}, 1 \mathrm{~s} 2$ $2 s 12 p 6 n l(n=3,4,5,6$; l=s, p, d, f, g and h). It calculated by using the fully relativistic atomic structure program FAC (flexible atomic code) adopted from [15]. We calculated the bound states of the atomic system in the configuration mixing approximation, by using a modified selfconsistent Dirac-Fock-Slater iteration to derive the radial orbitals for the construction of basis states. We present here the calculations of the atomic structure and energy levels of a Ne-like Ge. The energy levels of Ge XXIII are given in Table 1. The levels are placed according to ascending order of their energy. A comparison with the other data is also presented in Table 2. We concentrate our efforts on calculations of oscillator strengths for the configurations $1 \mathrm{~s} 22 \mathrm{~s} 22 \mathrm{p} 5 \mathrm{nl}, 1 \mathrm{~s} 2 \quad 2 \mathrm{~s} 12 \mathrm{p} 6 \mathrm{nl}$ $(n=3,4,5,6$; l=s, p, d, f, g and h). The weighted oscillator strengths $\mathrm{gf}_{\mathrm{ij}}[15]$ are given by

$$
\mathrm{gf}_{\mathrm{ij}}=\mathrm{L}^{-1} \omega(\alpha \omega)^{2 \mathrm{~L}-2} \mathrm{~S}_{\mathrm{ij}}
$$

where $\omega$ is the transition energy, $i$ is the statistical weight of the initial level of the transition (g) and $S_{i j}$ is the line strength given as

$$
S_{i j}=\left|\left\langle\psi_{i}\left\|O^{L}\right\| \psi_{j}\right\rangle\right|
$$

Here, $\mathrm{O}^{\mathrm{L}}$ denotes the spherical multipole operator of rank $L$ representing the interactions of electrons with the electromagnetic field, and $\psi_{i}$ and $\psi_{j}$ are the initial and final states of the transition, respectively. Weighted oscillator strength values are presented in Table 2. 


\section{Collision strengths and effective collision strengths}

The electron impact collision strengths have been calculated for transitions among the 241 levels at electron energies ranging from 10 to $20,000 \mathrm{eV}$. Tables (4) list the collision strengths at seven representative energies of 65.33, 1714.98, 2944.1, 4868.3, 7564.6, 11040 and $15221 \mathrm{eV}$. For brevity, the lower states are restricted to the lowest two levels in Tables (5). For Ne-like $\mathrm{Ge}$, close-coupling calculations have been carried out by Honglin Zhang and Douglas H. Sampson (1989) [14] to calculate the collision strengths for transitions among the lowest 88 levels within the $\mathrm{n}=3$ and 4 levels using HFS. In Table 3, we compare our collision strengths with their results at two scattered electron energies of 65.332 , and $1714.988 \mathrm{eV}$. Good agreement is found for the strong dipole-allowed transitions, especially at higher energies, and a maximum deviation about $9 \%$ between the two calculations. For some weak and forbidden transitions, however, the difference becomes evident. In the energy range shown above, there are no contributions of resonances. Therefore, the difference should not have been caused by the resonance effect. Recalling that we have included adequate partial wave summation, it cannot be attributed to a limited number of partial waves either. We suggest that the difference should primarily be attributed to the different $\mathrm{Cl}$ wavefunctions. As mentioned above, inclusion of more $\mathrm{Cl}$ leads to the decrease of the oscillator strengths, which scales directly to the collision strengths. The collision strength $\left(\Omega_{i j}\right)$ between an initial state " $i$ " and final state " $j$ " is defined in terms of the collision cross section $\left(\sigma_{i j}\right)[18]$ as

$$
\Omega_{i j}=\frac{\left(2 J_{i}+1\right) k_{i}^{2}}{\pi} \sigma_{i j}
$$

where $k_{i}^{2}$ is the channel energy of the continuum electron (Rydberg) and $j_{i}$ is the $J$-value of the initial level. In practical applications, one usually needs the excitation rate coefficients in the statistical equilibrium to obtain the populations of the levels. The rate coefficients can be obtained from the effective collision strength, which can be obtained by integrating the collision strength over Maxwellian distribution of electron velocities to give the effective collision strength $\left(Y_{i j}\right)$ [18]:

$$
\Upsilon_{i j}\left(T_{e}\right)=\int_{0}^{\infty} \Omega_{i j}\left(E_{j}\right) \exp \left(-E_{j} / k T_{e}\right) d\left(E_{j} / k T_{e}\right),
$$

where $E_{j}$ is the final kinetic energy of the electron, $T_{e}$ is the electron temperature, and $\mathrm{k}$ is Boltzmann's constant. If the collision strengths are assumed to be independent of the incident electron energy, we have $\gamma_{i j}=\Omega_{i j}$ The integration in Equation (5) should be carried out using energy-dependent collision strengths from threshold to infinity. The collision strengths at higher energies are particularly important for the dipole-allowed transitions, where they grow as $\ln (E)$. In the case of the electric quadrupole or magnetic-dipole transitions, the collision strengths are approximately constant, whereas for intercombination transitions they rapidly decrease as $1 / E^{2}$ in the asymptotic region. The collision strengths vary smoothly in high-energy region and the energy dependence of collision strengths for high energies can be properly accounted by using extrapolation technique. In astrophysical applications it is convenient to use excitation rate coefficients or thermally averaged collision strengths as a function of electron temperature. The excitation rates are obtained by averaging collision strengths over a Maxwellian distribution of electron energies. The collision excitation rate $C_{i j}$ in $\left(\mathrm{cm}^{3} \mathrm{~s}^{-1}\right)$ [19] can be obtained immediately from $Y_{i j}$ as follows:

$$
C_{i j}=\frac{8.629 \times 10^{-6}}{\omega_{i \sqrt{T_{e}}}} e^{-E_{i j} / k T_{e}} Y_{i j}
$$

Here $\omega_{i}$ is the statistical weight of level $i$ and $E_{i j}$ is the excitation energy. 


\section{Conclusion}

In this work the collision strengths, and effective collision strengths of Ge XXIII have been calculated using the fully relativistic FAC and compared to those from available theoretical calculations. Our results for the energy levels are in good agreement with those from the NIST database or other theoretical and experimental data. For strong transitions, the differences are within $1 \%, 12 \%$, and $19 \%$ compared with Honglin Zhang and Douglas H. Sampson work [14]. For weak transitions the differences are slightly larger. The different methods, the $\mathrm{Cl}$ involved, or the level mixing may be the reason for the differences between different works. For the weighted oscillator strengths and spontaneous transition rates we suggest that the present results can be used for plasma diagnostics. The collision strengths have been calculated using the distorted wave method in FAC, and the effective collision strengths are given for transitions among 241 levels at electron plasma temperatures $(650,850,1050$, $1250,1450,1650,1850)$ eV. To our knowledge, the present work is the first work of the lowest 241 levels including resonance contributions for electron impact excitation in Ge XXIII published in the literature. We expect that the present values of $\gamma$ are comparatively accurate to those from the largescale of calculations.

\section{References}

[1] P. Mazzotta, G. Mazzitelli, S. Colafrancesco, \& N. Vittorio. A\&AS. 133, 403 (1998)

[2] P. Bryans, N.R. Badnell, T.W.Gorczyca, J.M. Laming, W. Mitthumsiri, \& D.W. Savin. ApJ. 691, 1540 (2006)

[3] P. Bryans, E. Landi \& D.W. Savin. ApJS. 167, 343 (2009)

[4] D.L. Mathews, P.L. Hagelstein, M.D. Rosen, et al., Phys. Rev. Lett., 54, 110 (1985)

[5] F.G. Tomasel, J.J. Rocca, V.N. Shlyaptsev \& C.D. Macchietto, Phys. Rev. A. 55, 1437 (1997)

[6] P. Beiersdorfer, E. Behar, K.R.Boyce, et al. ApJ, 576, L169 (2002)

[7] P. Beiersdorfer, S.V.Goeler, M. Bitter \& D.B.

Thorn, Phys. Rev. A, 64, 032705 (2001)

[8] M.F. Gu, P. Beiersdorfer, G.V. Brown, et al. ApJ, 607, L143 (2004)

[9] B.W. Smith, J.C. Raymond, \& J.B. Mann. ApJ, 298, 898 (1985)

[10] G.X. Chen, A.K. Pradhan, \& W. Eissner, J. Phys. B: At. Mol. Opt. Phys., 36, 453 (2003)

[11] S.D. Loch, M.S.Pindzola, C.P. Ballance, \& D.C. Griffin, J. Phys. B: At. Mol. Opt. Phys., 39, 85 (2006)

[12] G.V. Brown, P. Beiersdorfer, H. Chen, et al. Phys. Rev. Lett., 96, 253201 (2006)

[13] H. L. Zhang and D. H. Sampson: At. Data Nucl. Data Tables 43 (1989) 1.

[14] HONG LIN ZHANG and DOUGLAS H. SAMPSON: At. Data Nucl. Data Tables 43, I-69 (1989).

[15] M.F. Gu, Astrophys. J. 582 (2003) 1241.

[16] A. Burgess, V.B. Sheorey, J. Phys. B 7 (1974) 2403.

[17] Abdelaziz, Wessameldin S. Optics and Photonics Journal, vol. 4, issue 09, pp. 246-269

[18] C. E. Hudson, C. A. Ramsbottom, and M. P. Scott: The Astrophysical Journal, 750:65 (7pp), 2012 May 1

[19] L. Di, J.R. Shi, G. Zhao, Data and Nucl. Data Tables 98 (2012) 437-480 


\section{Explanation of Tables}

Table 1: Comparison of some energy levels for Ge XXIII.

Table 2: Table 2. Comparison between some weighted Oscillator strengths for Ge XXIII.

Table 3: Comparison of present (FAC) collision strengths with other theoretical results from HFS [14] for transitions from the ground level of Ne-like Ge to the lowest 55 levels at two scattered electron energies of 65.332 , and $1714.988 \mathrm{eV} .1$

Table 4. Collision strengths at seven scattered electron energies of 65.33, 1714.98, 2944.1, 4868.3, 7564.6, 11040 and 15221eV for Ne-like Ge ions.

Table 5. The effective collision strengths at electron plasma temperatures $(650,850,1050$, 1250, 1450, 1650, 1850) eV for Ne-like Ge ions. 
Table 1. Comparison of some energy levels for Ge XXIII.

\begin{tabular}{|c|c|c|c|c|c|}
\hline Index & $\begin{array}{c}\text { State } \\
\text { Configuration }\end{array}$ & $\mathrm{J}^{\pi}$ & FAC & NIST & Exp. \\
\hline 1 & $2 p^{6}$ & $0^{e}$ & $0.00000 \mathrm{E}+00$ & $0.00000 E+00$ & $0.00000 E+00$ \\
\hline 2 & $2 p_{3 \backslash 2} 3 s_{1 \backslash 2}$ & $2^{\circ}$ & $1.23421 \mathrm{E}+03$ & & $1.236122 \mathrm{E}+03$ \\
\hline 3 & $2 p_{3 \mid 2} 3 s_{1 \mid 2}$ & $1^{\circ}$ & $1.23709 E+03$ & $1.23900 \mathrm{E}+03$ & $1.238602 \mathrm{E}+03$ \\
\hline 4 & $2 p_{1 \mid 2} 3 s_{1 \mid 2}$ & $0^{\circ}$ & $1.26664 \mathrm{E}+03$ & & $1.268358 \mathrm{E}+03$ \\
\hline 5 & $2 p_{1 \mid 2} 3 s_{1 \mid 2}$ & $1^{\circ}$ & $1.26822 \mathrm{E}+03$ & $1.27009 \mathrm{E}+03$ & $1.269598 \mathrm{E}+03$ \\
\hline 6 & $2 p_{3 \backslash 2} 3 p_{1 \backslash 2}$ & $1^{e}$ & $1.27690 \mathrm{E}+03$ & --- & $1.278277 \mathrm{E}+03$ \\
\hline 7 & $2 p_{3 \backslash 2} 3 p_{1 \backslash 2}$ & $2^{e}$ & $1.28046 \mathrm{E}+03$ & --- & $1.281997 \mathrm{E}+03$ \\
\hline 8 & $2 p_{3 \backslash 2} 3 p_{3 \backslash 2}$ & $3^{e}$ & $1.28642 \mathrm{E}+03$ & --- & $1.288196 \mathrm{E}+03$ \\
\hline 9 & $2 p_{3 \backslash 2} 3 p_{3 \backslash 2}$ & $1^{e}$ & $1.28726 \mathrm{E}+03$ & -- & $1.288196 \mathrm{E}+03$ \\
\hline 10 & $2 p_{3 \backslash 2} 3 p_{3 \backslash 2}$ & $2^{e}$ & $1.29059 E+03$ & $1.29204 \mathrm{E}+03$ & $1.291915 \mathrm{E}+03$ \\
\hline 11 & $2 p_{3 \mid 2} 3 p_{3 \backslash 2}$ & $0^{e}$ & $1.30216 \mathrm{E}+03$ & -- & $1.303074 \mathrm{E}+03$ \\
\hline 12 & $2 p_{1 \mid 2} 3 p_{1 \mid 2}$ & $1^{e}$ & $1.31186 \mathrm{E}+03$ & --- & $1.314232 \mathrm{E}+03$ \\
\hline 13 & $2 p_{1 \mid 2} 3 p_{3 \backslash 2}$ & $1^{e}$ & $1.31985 \mathrm{E}+03$ & --- & $1.321671 E+03$ \\
\hline 14 & $2 p_{1 \mid 2} 3 p_{3 \backslash 2}$ & $2^{e}$ & $1.32083 \mathrm{E}+03$ & $1.32254 \mathrm{E}+03$ & $1.322911 \mathrm{E}+03$ \\
\hline 15 & $2 p_{1 \mid 2} 3 p_{1 \mid 2}$ & $0^{e}$ & $1.33447 E+03$ & $1.33333 \mathrm{E}+03$ & $1.332830 E+03$ \\
\hline 16 & $2 p_{3 \backslash 2} 3 d_{3 \backslash 2}$ & $0^{\circ}$ & $1.34070 \mathrm{E}+03$ & --- & $1.342749 \mathrm{E}+03$ \\
\hline 17 & $2 p_{3 \backslash 2} 3 d_{3 \backslash 2}$ & $1^{\circ}$ & $1.34247 E+03$ & $1.34473 \mathrm{E}+03$ & $1.343989 \mathrm{E}+03$ \\
\hline 18 & $2 p_{3 \mid 2} 3 d_{5 \mid 2}$ & $4^{\circ}$ & $1.34553 \mathrm{E}+03$ & --- & --- \\
\hline 19 & $2 p_{3 \backslash 2} 3 d_{5 \backslash 2}$ & $2^{\circ}$ & $1.34575 \mathrm{E}+03$ & --- & $1.347708 \mathrm{E}+03$ \\
\hline 20 & $2 p_{3 \backslash 2} 3 d_{3 \backslash 2}$ & $3^{\circ}$ & $1.34594 \mathrm{E}+03$ & --- & $1.347708 \mathrm{E}+03$ \\
\hline 21 & $2 p_{3 \backslash 2} 3 d_{3 \backslash 2}$ & $2^{\circ}$ & $1.34871 \mathrm{E}+03$ & --- & $1.350188 \mathrm{E}+03$ \\
\hline 22 & $2 p_{3 \backslash 2} 3 d_{5 \backslash 2}$ & $3^{\circ}$ & $1.35077 \mathrm{E}+03$ & --- & $1.352668 \mathrm{E}+03$ \\
\hline 23 & $2 p_{3 \backslash 2} 3 d_{5 \backslash 2}$ & $1^{\circ}$ & $1.35938 \mathrm{E}+03$ & $1.36085 \mathrm{E}+03$ & $1.361346 \mathrm{E}+03$ \\
\hline 24 & $2 p_{1 \mid 2} 3 d_{3 \mid 2}$ & $2^{\circ}$ & $1.37811 \mathrm{E}+03$ & --- & --- \\
\hline 25 & $2 p_{1 \backslash 2} 3 d_{5 \backslash 2}$ & $2^{\circ}$ & $1.37971 \mathrm{E}+03$ & --- & --- \\
\hline
\end{tabular}


Table 2. Comparison between some weighted Oscillator strengths for Ge XXIII.

\begin{tabular}{|ccccc|}
\hline $\mathrm{j}$ & $\mathrm{i}$ & $\mathrm{gf}(\mathrm{FAC})$ & $\mathrm{gf}(\mathrm{CIV} 3)$ & gf $(\mathrm{RCI})$ \\
\hline 3 & 1 & $1.29 \mathrm{E}-01$ & $1.280 \mathrm{E}-01$ & ---- \\
5 & 1 & $8.88 \mathrm{E}-02$ & $8.480 \mathrm{E}-02$ & ---- \\
6 & 2 & $2.26 \mathrm{E}-01$ & $2.257 \mathrm{E}-01$ & 0.2685 \\
7 & 2 & $2.13 \mathrm{E}-01$ & $2.113 \mathrm{E}-01$ & 0.2246 \\
7 & 3 & $2.15 \mathrm{E}-01$ & $2.185 \mathrm{E}-01$ & 0.2204 \\
8 & 2 & $7.00 \mathrm{E}-01$ & $7.002 \mathrm{E}-01$ & 0.7275 \\
9 & 2 & $4.53 \mathrm{E}-04$ & $4.000 \mathrm{E}-04$ & 0.0001 \\
9 & 3 & $2.84 \mathrm{E}-01$ & $2.827 \mathrm{E}-01$ & 0.2914 \\
10 & 2 & $2.91 \mathrm{E}-01$ & $2.923 \mathrm{E}-01$ & 0.3092 \\
10 & 3 & $2.52 \mathrm{E}-01$ & $2.480 \mathrm{E}-01$ & 0.2431 \\
10 & 5 & $6.21 \mathrm{E}-04$ & $5.000 \mathrm{E}-04$ & ---- \\
11 & 3 & $1.08 \mathrm{E}-01$ & $1.102 \mathrm{E}-01$ & 0.0930 \\
11 & 5 & $1.26 \mathrm{E}-02$ & $1.130 \mathrm{E}-02$ & ---- \\
12 & 2 & $6.08 \mathrm{E}-04$ & $7.000 \mathrm{E}-04$ & 0.0000 \\
12 & 3 & $5.51 \mathrm{E}-04$ & $7.000 \mathrm{E}-04$ & 0.0076 \\
12 & 4 & $8.97 \mathrm{E}-02$ & $8.920 \mathrm{E}-02$ & 0.1111 \\
12 & 5 & $1.59 \mathrm{E}-01$ & $1.601 \mathrm{E}-01$ & 0.1542 \\
13 & 2 & $1.24 \mathrm{E}-02$ & $1.300 \mathrm{E}-02$ & ---- \\
13 & 3 & $2.86 \mathrm{E}-04$ & $4.000 \mathrm{E}-04$ & ---- \\
13 & 4 & $1.96 \mathrm{E}-01$ & $1.952 \mathrm{E}-01$ & 0.2017 \\
13 & 5 & $9.70 \mathrm{E}-02$ & $9.450 \mathrm{E}-02$ & 0.1224 \\
14 & 2 & $1.78 \mathrm{E}-03$ & $1.800 \mathrm{E}-03$ & ---- \\
14 & 3 & $1.08 \mathrm{E}-03$ & $1.000 \mathrm{E}-03$ & ---- \\
14 & 5 & $5.08 \mathrm{E}-01$ & $5.074 \mathrm{E}-01$ & 0.5590 \\
15 & 3 & $5.19 \mathrm{E}-02$ & $4.470 \mathrm{E}-02$ & --- \\
15 & 5 & $1.15 \mathrm{E}-01$ & $1.125 \mathrm{E}-01$ & 0.1002 \\
\hline
\end{tabular}


Comparison of present (FAC) collision strengths with other theoretical results from HFS [14] for transitions from the ground level of $\mathrm{Ne}$-like Ge to the lowest 55 levels at two scattered electron energies of 65.332, and $1714.988 \mathrm{eV} .1$

\begin{tabular}{|c|c|c|c|c|c|c|c|}
\hline \multirow{2}{*}{1} & \multirow[t]{2}{*}{ J } & \multirow{2}{*}{ State Configuration } & \multirow[t]{2}{*}{$\begin{array}{c}\text { state symbol } \\
\text { HFS[14] }\end{array}$} & \multicolumn{2}{|l|}{ this work } & \multicolumn{2}{|l|}{ HFS [14] } \\
\hline & & & & 65.332 & 1714.988 & 65.332 & 1714.988 \\
\hline 1 & 2 & $\left(2 p_{3 / 2} 3 s_{1 / 2}\right)_{2}$ & L1 & $9.89 \mathrm{E}-04$ & $4.27 \mathrm{E}-04$ & 9.67E-04 & $4.04 \mathrm{E}-04$ \\
\hline 1 & 3 & $\left(2 p_{3 / 2} 3 s_{1 / 2}\right)_{1}$ & K1 & $1.09 \mathrm{E}-03$ & $2.90 \mathrm{E}-03$ & $1.14 \mathrm{E}-03$ & $3.20 \mathrm{E}-03$ \\
\hline 1 & 4 & $\left(2 \mathrm{p}_{1 / 2} 3 \mathrm{~s}_{1 / 2}\right)_{0}$ & J1 & $1.93 \mathrm{E}-04$ & 8.41E-05 & $1.97 \mathrm{E}-04$ & $8.15 \mathrm{E}-05$ \\
\hline 1 & 5 & $\left(2 \mathrm{p}_{1 / 2} 3 \mathrm{~s}_{1 / 2}\right)_{1}$ & K2 & $9.01 \mathrm{E}-04$ & $1.95 \mathrm{E}-03$ & $9.43 \mathrm{E}-04$ & $2.16 \mathrm{E}-03$ \\
\hline 1 & 6 & $\left(2 p_{3 / 2} 3 p_{1 / 2}\right)_{1}$ & E1 & $2.30 \mathrm{E}-03$ & $9.28 \mathrm{E}-04$ & $2.34 \mathrm{E}-03$ & $9.18 \mathrm{E}-04$ \\
\hline 1 & 7 & $\left(2 p_{3 / 2} 3 p_{1 / 2}\right)_{2}$ & F1 & $2.38 \mathrm{E}-03$ & $2.09 \mathrm{E}-03$ & $2.42 \mathrm{E}-03$ & $2.14 \mathrm{E}-03$ \\
\hline 1 & 8 & $\left(2 p_{3 / 2} 3 p_{3 / 2}\right)_{3}$ & G1 & $2.86 \mathrm{E}-03$ & $1.02 \mathrm{E}-03$ & $2.84 \mathrm{E}-03$ & $9.82 \mathrm{E}-04$ \\
\hline 1 & 9 & $\left(2 p_{3 / 2} 3 p_{3 / 2}\right)_{1}$ & E2 & $1.20 \mathrm{E}-03$ & 4.39E-04 & $1.17 \mathrm{E}-03$ & 4.13E-04 \\
\hline 1 & 10 & $\left(2 p_{3 / 2} 3 p_{3 / 2}\right)_{2}$ & F2 & $1.89 \mathrm{E}-03$ & $1.83 \mathrm{E}-03$ & $1.92 \mathrm{E}-03$ & $1.90 \mathrm{E}-03$ \\
\hline 1 & 11 & $\left(2 p_{3 / 2} 3 p_{3 / 2}\right)_{0}$ & D1 & $5.11 \mathrm{E}-03$ & $5.62 \mathrm{E}-03$ & $4.66 \mathrm{E}-03$ & $5.12 \mathrm{E}-03$ \\
\hline 1 & 12 & $\left(2 p_{1 / 2} 3 p_{1 / 2}\right)_{1}$ & E3 & $1.14 \mathrm{E}-03$ & $4.11 \mathrm{E}-04$ & $1.15 \mathrm{E}-03$ & 4.00E-04 \\
\hline 1 & 13 & $\left(2 p_{1 / 2} 3 p_{3 / 2}\right)_{1}$ & E4 & $1.29 \mathrm{E}-03$ & $4.86 \mathrm{E}-04$ & $1.29 \mathrm{E}-03$ & 4.69E-04 \\
\hline 1 & 14 & $\left(2 p_{1 / 2} 3 p_{3 / 2}\right)_{2}$ & F3 & $2.20 \mathrm{E}-03$ & $1.99 \mathrm{E}-03$ & $2.25 \mathrm{E}-03$ & $2.06 \mathrm{E}-03$ \\
\hline 1 & 15 & $\left(2 p_{1 / 2} 3 p_{1 / 2}\right)_{0}$ & D2 & $2.32 \mathrm{E}-02$ & $2.62 \mathrm{E}-02$ & $2.28 \mathrm{E}-02$ & $2.58 \mathrm{E}-02$ \\
\hline 1 & 16 & $\left(2 p_{3 / 2} 3 d_{3 / 2}\right)_{0}$ & $\mathrm{~J} 2$ & $1.33 \mathrm{E}-03$ & 4.40E-04 & $1.30 \mathrm{E}-03$ & $4.12 \mathrm{E}-04$ \\
\hline 1 & 17 & $\left(2 p_{3 / 2} 3 d_{3 / 2}\right)_{1}$ & K3 & $3.81 \mathrm{E}-03$ & $1.58 \mathrm{E}-03$ & $3.70 \mathrm{E}-03$ & $1.48 \mathrm{E}-03$ \\
\hline 1 & 18 & $\left(2 p_{3 / 2} 3 d_{5 / 2}\right)_{4}$ & N1 & $4.46 \mathrm{E}-03$ & $1.33 \mathrm{E}-03$ & $4.38 \mathrm{E}-03$ & $1.26 \mathrm{E}-03$ \\
\hline 1 & 19 & $\left(2 p_{3 / 2} 3 d_{5 / 2}\right)_{2}$ & L2 & $4.51 \mathrm{E}-03$ & $1.43 \mathrm{E}-03$ & $4.45 \mathrm{E}-03$ & $1.36 \mathrm{E}-03$ \\
\hline 1 & 20 & $\left(2 p_{3 / 2} 3 d_{3 / 2}\right)_{3}$ & M1 & $3.10 \mathrm{E}-03$ & $2.00 \mathrm{E}-03$ & $3.07 \mathrm{E}-03$ & 2.03E-03 \\
\hline 1 & 21 & $\left(2 p_{3 / 2} 3 d_{3 / 2}\right)_{2}$ & L3 & $1.82 \mathrm{E}-03$ & $4.85 \mathrm{E}-04$ & $1.75 \mathrm{E}-03$ & 4.43E-04 \\
\hline 1 & 22 & $\left(2 p_{3 / 2} 3 d_{5 / 2}\right)_{3}$ & M2 & $2.15 \mathrm{E}-03$ & $1.62 \mathrm{E}-03$ & $2.11 \mathrm{E}-03$ & $1.64 \mathrm{E}-03$ \\
\hline 1 & 23 & $\left(2 p_{3 / 2} 3 d_{5 / 2}\right)_{1}$ & K4 & $2.86 \mathrm{E}-02$ & $5.02 \mathrm{E}-02$ & $2.86 \mathrm{E}-02$ & $5.11 \mathrm{E}-02$ \\
\hline 1 & 24 & $\left(2 p_{1 / 2} 3 d_{3 / 2}\right)_{2}$ & L4 & $2.12 \mathrm{E}-03$ & $6.20 \mathrm{E}-04$ & 2.09E-03 & $5.83 \mathrm{E}-04$ \\
\hline 1 & 25 & $\left(2 p_{1 / 2} 3 d_{5 / 2}\right)_{2}$ & L5 & $3.10 \mathrm{E}-03$ & $9.49 \mathrm{E}-04$ & $3.04 \mathrm{E}-03$ & $8.96 \mathrm{E}-04$ \\
\hline 1 & 26 & $\left(2 p_{1 / 2} 3 d_{5 / 2}\right)_{3}$ & M3 & $2.62 \mathrm{E}-03$ & $1.90 \mathrm{E}-03$ & $2.61 \mathrm{E}-03$ & $1.93 \mathrm{E}-03$ \\
\hline 1 & 27 & $\left(2 p_{1 / 2} 3 d_{3 / 2}\right)_{1}$ & K5 & $4.84 \mathrm{E}-02$ & $8.63 \mathrm{E}-02$ & $4.75 \mathrm{E}-02$ & $8.64 \mathrm{E}-02$ \\
\hline 1 & 28 & $\left(2 \mathrm{~s}_{1 / 2} 3 \mathrm{~s}_{1 / 2}\right)_{1}$ & E5 & $6.76 \mathrm{E}-04$ & 2.32E-04 & $6.59 \mathrm{E}-04$ & $2.20 \mathrm{E}-04$ \\
\hline 1 & 29 & $\left(2 \mathrm{~s}_{1 / 2} 3 \mathrm{~s}_{1 / 2}\right)_{0}$ & D3 & $1.02 \mathrm{E}-02$ & $1.21 \mathrm{E}-02$ & $9.69 \mathrm{E}-03$ & $1.15 \mathrm{E}-02$ \\
\hline 1 & 30 & $\left(2 s_{1 / 2} 3 p_{1 / 2}\right)_{0}$ & $\mathrm{~J} 3$ & $1.69 \mathrm{E}-04$ & 7.02E-05 & $1.67 \mathrm{E}-04$ & $6.73 \mathrm{E}-05$ \\
\hline 1 & 31 & $\left(2 s_{1 / 2} 3 p_{1 / 2}\right)_{1}$ & K6 & 7.57E-04 & $1.34 \mathrm{E}-03$ & $7.60 \mathrm{E}-04$ & $1.33 \mathrm{E}-03$ \\
\hline 1 & 32 & $\left(2 s_{1 / 2} 3 p_{3 / 2}\right)_{2}$ & L6 & $8.16 \mathrm{E}-04$ & $3.45 \mathrm{E}-04$ & 7.93E-04 & 3.27E-04 \\
\hline 1 & 33 & $\left(2 s_{1 / 2} 3 p_{3 / 2}\right)_{1}$ & K7 & $1.55 \mathrm{E}-03$ & $4.91 \mathrm{E}-03$ & $1.51 \mathrm{E}-03$ & $4.91 \mathrm{E}-03$ \\
\hline 1 & 34 & $\left(2 s_{1 / 2} 3 d_{3 / 2}\right)_{1}$ & E6 & $1.21 \mathrm{E}-03$ & $4.28 \mathrm{E}-04$ & $1.19 \mathrm{E}-03$ & 4.09E-04 \\
\hline 1 & 35 & $\left(2 \mathrm{~s}_{1 / 2} 3 \mathrm{~d}_{3 / 2}\right)_{2}$ & F4 & $2.08 \mathrm{E}-03$ & $8.60 \mathrm{E}-04$ & $2.05 \mathrm{E}-03$ & $8.28 \mathrm{E}-04$ \\
\hline 1 & 36 & $\left(2 s_{1 / 2} 3 d_{5 / 2}\right)_{3}$ & G2 & $2.80 \mathrm{E}-03$ & $9.86 \mathrm{E}-04$ & $2.76 \mathrm{E}-03$ & $9.43 \mathrm{E}-04$ \\
\hline 1 & 37 & $\left(2 s_{1 / 2} 3 d_{5 / 2}\right)_{2}$ & F5 & $9.86 \mathrm{E}-03$ & $1.73 \mathrm{E}-02$ & $9.76 \mathrm{E}-03$ & $1.76 \mathrm{E}-02$ \\
\hline 1 & 38 & $\left(2 p_{3 / 2} 4 s_{1 / 2}\right)_{2}$ & L7 & 3.14E-04 & $1.36 \mathrm{E}-04$ & $3.11 \mathrm{E}-04$ & $1.32 \mathrm{E}-04$ \\
\hline 1 & 39 & $\left(2 p_{3 / 2} 4 s_{1 / 2}\right)_{1}$ & K8 & $1.82 \mathrm{E}-04$ & 3.79E-04 & $2.04 \mathrm{E}-04$ & $4.52 \mathrm{E}-04$ \\
\hline 1 & 40 & $\left(2 p_{3 / 2} 4 p_{1 / 2}\right)_{1}$ & E7 & $6.01 \mathrm{E}-04$ & 2.43E-04 & $6.25 \mathrm{E}-04$ & 2.47E-04 \\
\hline 1 & 41 & $\left(2 p_{3 / 2} 4 p_{1 / 2}\right)_{2}$ & F6 & $6.62 \mathrm{E}-04$ & $4.52 \mathrm{E}-04$ & $6.76 \mathrm{E}-04$ & 4.66E-04 \\
\hline 1 & 42 & $\left(2 p_{3 / 2} 4 p_{3 / 2}\right)_{3}$ & G3 & $9.70 \mathrm{E}-04$ & 3.50E-04 & $9.71 \mathrm{E}-04$ & $3.42 \mathrm{E}-04$ \\
\hline 1 & 43 & $\left(2 p_{3 / 2} 4 p_{3 / 2}\right)_{1}$ & E8 & 4.00E-04 & $1.48 \mathrm{E}-04$ & 3.97E-04 & $1.42 \mathrm{E}-04$ \\
\hline 1 & 44 & $\left(2 p_{3 / 2} 4 p_{3 / 2}\right)_{2}$ & F7 & $5.19 \mathrm{E}-04$ & $3.73 \mathrm{E}-04$ & $5.28 \mathrm{E}-04$ & 3.86E-04 \\
\hline 1 & 45 & $\left(2 p_{3 / 2} 4 p_{3 / 2}\right)_{0}$ & D4 & 3.86E-03 & $4.42 \mathrm{E}-03$ & $3.60 \mathrm{E}-03$ & $4.11 \mathrm{E}-03$ \\
\hline 1 & 46 & $\left(2 \mathrm{p}_{1 / 2} 4 \mathrm{~s}_{1 / 2}\right)_{0}$ & J4 & $6.27 \mathrm{E}-05$ & 2.70E-05 & 6.47E-05 & $2.69 \mathrm{E}-05$ \\
\hline 1 & 47 & $\left(2 \mathrm{p}_{1 / 2} 4 \mathrm{~s}_{1 / 2}\right)_{1}$ & K9 & $2.21 \mathrm{E}-04$ & 3.59E-04 & 2.47E-04 & 4.14E-04 \\
\hline 1 & 48 & $\left(2 p_{3 / 2} 4 d_{3 / 2}\right)_{0}$ & $\mathrm{~J} 5$ & 4.27E-04 & $1.46 \mathrm{E}-04$ & 4.34E-04 & $1.42 \mathrm{E}-04$ \\
\hline 1 & 49 & $\left(2 p_{3 / 2} 4 d_{3 / 2}\right)_{1}$ & K10 & $1.15 \mathrm{E}-03$ & 4.56E-04 & $1.15 \mathrm{E}-03$ & 4.44E-04 \\
\hline 1 & 50 & $\left(2 p_{3 / 2} 4 d_{5 / 2}\right)_{4}$ & N2 & $1.50 \mathrm{E}-03$ & $4.66 \mathrm{E}-04$ & $1.51 \mathrm{E}-03$ & 4.50E-04 \\
\hline 1 & 51 & $\left(2 p_{3 / 2} 4 d_{3 / 2}\right)_{3}$ & M4 & $8.85 \mathrm{E}-04$ & 4.52E-04 & $8.83 \mathrm{E}-04$ & 4.54E-04 \\
\hline 1 & 52 & $\left(2 p_{3 / 2} 4 d_{5 / 2}\right)_{2}$ & L8 & $1.30 \mathrm{E}-03$ & $4.18 \mathrm{E}-04$ & $1.34 \mathrm{E}-03$ & $4.12 \mathrm{E}-04$ \\
\hline 1 & 53 & $\left(2 p_{3 / 2} 4 d_{3 / 2}\right)_{2}$ & L9 & $6.80 \mathrm{E}-04$ & $1.91 \mathrm{E}-04$ & $6.60 \mathrm{E}-04$ & $1.75 \mathrm{E}-04$ \\
\hline 1 & 54 & $\left(2 p_{3 / 2} 4 d_{5 / 2}\right)_{3}$ & M5 & $6.45 \mathrm{E}-04$ & $3.23 \mathrm{E}-04$ & $6.37 \mathrm{E}-04$ & $3.22 \mathrm{E}-04$ \\
\hline 1 & 55 & $\left(2 p_{3 / 2} 4 d_{5 / 2}\right)_{1}$ & K11 & $1.03 \mathrm{E}-02$ & $1.75 \mathrm{E}-02$ & 9.99E-03 & $1.72 \mathrm{E}-02$ \\
\hline
\end{tabular}


Table 4. Collision strengths at seven scattered electron energies of $65.33,1714.98,2944.1,4868.3$, 7564.6, 11040 and $15221 \mathrm{eV}$ for Ne-like Ge ions.

\begin{tabular}{|c|c|c|c|c|c|c|c|c|}
\hline \multirow[b]{2}{*}{$\mathrm{i}$} & \multirow[b]{2}{*}{ j } & \multicolumn{7}{|c|}{ collision strengths at energies } \\
\hline & & $65.33 \mathrm{eV}$ & $1714.98 \mathrm{eV}$ & $2944.1 \mathrm{eV}$ & $4868.3 \mathrm{eV}$ & $7564.6 \mathrm{eV}$ & $11040 \mathrm{eV}$ & $15221 \mathrm{eV}$ \\
\hline \multirow[t]{66}{*}{1} & 2 & $4.04 \mathrm{E}-04$ & $9.67 \mathrm{E}-04$ & $1.65 \mathrm{E}-04$ & 7.33E-05 & $3.58 \mathrm{E}-05$ & $1.93 \mathrm{E}-05$ & $0.00 \mathrm{E}+00$ \\
\hline & 3 & $3.20 \mathrm{E}-03$ & 1.14E-03 & $5.66 \mathrm{E}-03$ & 8.01E-03 & $1.02 \mathrm{E}-02$ & $1.21 \mathrm{E}-02$ & 2.51E-02 \\
\hline & 4 & 8.15E-05 & 1.97E-04 & 3.34E-05 & $1.48 \mathrm{E}-05$ & $7.25 \mathrm{E}-06$ & $3.92 \mathrm{E}-06$ & $0.00 E+00$ \\
\hline & 5 & 2.16E-03 & 9.43E-04 & 3.73E-03 & $5.26 \mathrm{E}-03$ & 6.70E-03 & 7.98E-03 & $1.67 \mathrm{E}-02$ \\
\hline & 6 & $9.18 \mathrm{E}-04$ & $2.34 \mathrm{E}-03$ & $3.81 \mathrm{E}-04$ & $1.74 \mathrm{E}-04$ & $8.75 \mathrm{E}-05$ & 4.85E-05 & $0.00 \mathrm{E}+00$ \\
\hline & 7 & 2.14E-03 & $2.42 \mathrm{E}-03$ & $2.32 \mathrm{E}-03$ & $2.54 \mathrm{E}-03$ & 2.72E-03 & 2.87E-03 & $2.85 \mathrm{E}-03$ \\
\hline & 8 & $9.82 \mathrm{E}-04$ & 2.84E-03 & 3.69E-04 & $1.55 \mathrm{E}-04$ & 7.25E-05 & 3.79E-05 & $0.00 \mathrm{E}+00$ \\
\hline & 9 & 4.13E-04 & 1.17E-03 & $1.54 \mathrm{E}-04$ & $6.48 \mathrm{E}-05$ & 3.07E-05 & $1.62 \mathrm{E}-05$ & $0.00 \mathrm{E}+00$ \\
\hline & 10 & $1.90 \mathrm{E}-03$ & $1.92 \mathrm{E}-03$ & 2.17E-03 & $2.43 \mathrm{E}-03$ & 2.63E-03 & $2.78 \mathrm{E}-03$ & 2.79E-03 \\
\hline & 11 & 5.12E-03 & 4.66E-03 & 5.35E-03 & $5.48 \mathrm{E}-03$ & $5.58 \mathrm{E}-03$ & $5.68 \mathrm{E}-03$ & 5.34E-03 \\
\hline & 12 & 4.00E-04 & $1.15 \mathrm{E}-03$ & $1.48 \mathrm{E}-04$ & 6.15E-05 & $2.86 \mathrm{E}-05$ & 1.49E-05 & $0.00 E+00$ \\
\hline & 13 & 4.69E-04 & $1.29 \mathrm{E}-03$ & $1.80 \mathrm{E}-04$ & 7.71E-05 & 3.71E-05 & $1.99 \mathrm{E}-05$ & $0.00 \mathrm{E}+00$ \\
\hline & 14 & $2.06 \mathrm{E}-03$ & $2.25 \mathrm{E}-03$ & $2.29 \mathrm{E}-03$ & $2.53 \mathrm{E}-03$ & $2.73 \mathrm{E}-03$ & $2.88 \mathrm{E}-03$ & $2.90 \mathrm{E}-03$ \\
\hline & 15 & $2.58 \mathrm{E}-02$ & $2.28 \mathrm{E}-02$ & $2.72 \mathrm{E}-02$ & $2.80 \mathrm{E}-02$ & $2.85 \mathrm{E}-02$ & 2.90E-02 & $2.74 \mathrm{E}-02$ \\
\hline & 16 & 4.12E-04 & $1.30 \mathrm{E}-03$ & $1.45 \mathrm{E}-04$ & 5.85E-05 & 2.69E-05 & 1.37E-05 & $0.00 E+00$ \\
\hline & 17 & $1.48 \mathrm{E}-03$ & 3.70E-03 & 9.35E-04 & 8.59E-04 & $9.16 \mathrm{E}-04$ & $1.01 \mathrm{E}-03$ & $1.86 \mathrm{E}-03$ \\
\hline & 18 & $1.26 \mathrm{E}-03$ & 4.38E-03 & 4.08E-04 & $1.58 \mathrm{E}-04$ & 7.11E-05 & $3.58 \mathrm{E}-05$ & $0.00 E+00$ \\
\hline & 19 & $1.36 \mathrm{E}-03$ & $4.45 \mathrm{E}-03$ & 4.66E-04 & $1.85 \mathrm{E}-04$ & 8.45E-05 & 4.30E-05 & $0.00 E+00$ \\
\hline & 20 & 2.03E-03 & 3.07E-03 & $1.96 \mathrm{E}-03$ & 2.05E-03 & 2.17E-03 & 2.27E-03 & 2.34E-03 \\
\hline & 21 & 4.43E-04 & $1.75 \mathrm{E}-03$ & $1.28 \mathrm{E}-04$ & 4.50E-05 & $1.93 \mathrm{E}-05$ & 9.83E-06 & $0.00 E+00$ \\
\hline & 22 & $1.64 \mathrm{E}-03$ & $2.11 \mathrm{E}-03$ & 1.77E-03 & $1.94 \mathrm{E}-03$ & 2.07E-03 & $2.18 \mathrm{E}-03$ & $2.28 \mathrm{E}-03$ \\
\hline & 23 & 5.11E-02 & $2.86 \mathrm{E}-02$ & 7.37E-02 & $9.44 \mathrm{E}-02$ & $1.13 \mathrm{E}-01$ & $1.31 \mathrm{E}-01$ & 2.43E-01 \\
\hline & 24 & 5.83E-04 & 2.09E-03 & $1.83 \mathrm{E}-04$ & $6.89 \mathrm{E}-05$ & 3.07E-05 & $1.55 \mathrm{E}-05$ & $0.00 E+00$ \\
\hline & 25 & 8.96E-04 & $3.04 \mathrm{E}-03$ & $2.96 \mathrm{E}-04$ & $1.14 \mathrm{E}-04$ & 5.15E-05 & $2.62 \mathrm{E}-05$ & $0.00 E+00$ \\
\hline & 26 & 1.93E-03 & 2.61E-03 & $1.98 \mathrm{E}-03$ & 2.13E-03 & $2.26 \mathrm{E}-03$ & 2.37E-03 & 2.47E-03 \\
\hline & 27 & 8.65E-02 & 4.76E-02 & $1.25 \mathrm{E}-01$ & $1.61 \mathrm{E}-01$ & $1.94 \mathrm{E}-01$ & 2.23E-01 & 4.17E-01 \\
\hline & 28 & $2.20 \mathrm{E}-04$ & $6.59 \mathrm{E}-04$ & $8.62 \mathrm{E}-05$ & $3.92 \mathrm{E}-05$ & $1.98 \mathrm{E}-05$ & $1.09 \mathrm{E}-05$ & $0.00 E+00$ \\
\hline & 29 & 1.15E-02 & $9.69 \mathrm{E}-03$ & $1.24 \mathrm{E}-02$ & $1.29 \mathrm{E}-02$ & $1.33 \mathrm{E}-02$ & $1.36 \mathrm{E}-02$ & 1.31E-02 \\
\hline & 30 & 6.73E-05 & 1.67E-04 & $2.72 \mathrm{E}-05$ & $1.18 \mathrm{E}-05$ & $5.55 \mathrm{E}-06$ & $2.84 \mathrm{E}-06$ & $0.00 E+00$ \\
\hline & 31 & $1.33 \mathrm{E}-03$ & 7.61E-04 & 2.19E-03 & 3.09E-03 & $3.95 \mathrm{E}-03$ & 4.75E-03 & $1.03 \mathrm{E}-02$ \\
\hline & 32 & $3.27 \mathrm{E}-04$ & 7.93E-04 & $1.34 \mathrm{E}-04$ & $5.85 E-05$ & 2.77E-05 & $1.44 \mathrm{E}-05$ & $0.00 E+00$ \\
\hline & 33 & 4.91E-03 & $1.51 \mathrm{E}-03$ & $9.01 \mathrm{E}-03$ & $1.31 \mathrm{E}-02$ & $1.69 \mathrm{E}-02$ & $2.03 \mathrm{E}-02$ & 4.48E-02 \\
\hline & 34 & 4.09E-04 & 1.19E-03 & $1.52 \mathrm{E}-04$ & $6.49 \mathrm{E}-05$ & $3.12 \mathrm{E}-05$ & $1.63 \mathrm{E}-05$ & $0.00 E+00$ \\
\hline & 35 & $8.28 \mathrm{E}-04$ & 2.05E-03 & 4.57E-04 & $3.46 \mathrm{E}-04$ & $3.12 \mathrm{E}-04$ & $3.04 \mathrm{E}-04$ & $2.70 \mathrm{E}-04$ \\
\hline & 36 & $9.43 \mathrm{E}-04$ & $2.76 \mathrm{E}-03$ & $3.51 \mathrm{E}-04$ & $1.49 \mathrm{E}-04$ & 7.17E-05 & $3.76 \mathrm{E}-05$ & $0.00 E+00$ \\
\hline & 37 & $1.76 \mathrm{E}-02$ & $9.76 \mathrm{E}-03$ & $2.31 \mathrm{E}-02$ & 2.69E-02 & $2.93 \mathrm{E}-02$ & $3.12 \mathrm{E}-02$ & 3.11E-02 \\
\hline & 38 & $1.32 \mathrm{E}-04$ & $3.11 \mathrm{E}-04$ & $5.43 \mathrm{E}-05$ & $2.42 \mathrm{E}-05$ & 1.19E-05 & $6.40 \mathrm{E}-06$ & $0.00 E+00$ \\
\hline & 39 & $4.52 \mathrm{E}-04$ & 2.05E-04 & 7.76E-04 & $1.10 \mathrm{E}-03$ & $1.39 \mathrm{E}-03$ & $1.66 \mathrm{E}-03$ & 3.37E-03 \\
\hline & 40 & 2.47E-04 & $6.25 \mathrm{E}-04$ & $1.02 \mathrm{E}-04$ & 4.63E-05 & $2.32 \mathrm{E}-05$ & $1.27 \mathrm{E}-05$ & $0.00 E+00$ \\
\hline & 41 & 4.66E-04 & $6.76 \mathrm{E}-04$ & 4.50E-04 & 4.85E-04 & $5.18 \mathrm{E}-04$ & $5.46 \mathrm{E}-04$ & 5.47E-04 \\
\hline & 42 & $3.42 \mathrm{E}-04$ & $9.71 \mathrm{E}-04$ & $1.29 \mathrm{E}-04$ & 5.47E-05 & $2.58 \mathrm{E}-05$ & $1.34 \mathrm{E}-05$ & $0.00 \mathrm{E}+00$ \\
\hline & 43 & $1.42 \mathrm{E}-04$ & 3.97E-04 & 5.34E-05 & $2.26 \mathrm{E}-05$ & 1.07E-05 & $5.68 \mathrm{E}-06$ & $0.00 E+00$ \\
\hline & 44 & $3.86 \mathrm{E}-04$ & $5.28 \mathrm{E}-04$ & $3.93 \mathrm{E}-04$ & 4.36E-04 & 4.71E-04 & $5.00 \mathrm{E}-04$ & 5.07E-04 \\
\hline & 45 & 4.11E-03 & 3.60E-03 & 4.38E-03 & 4.53E-03 & 4.64E-03 & 4.73E-03 & 4.47E-03 \\
\hline & 46 & 2.69E-05 & 6.47E-05 & $1.09 \mathrm{E}-05$ & 4.81E-06 & $2.35 \mathrm{E}-06$ & $1.25 \mathrm{E}-06$ & $0.00 E+00$ \\
\hline & 47 & 4.14E-04 & $2.47 \mathrm{E}-04$ & $6.63 \mathrm{E}-04$ & $9.25 \mathrm{E}-04$ & $1.16 \mathrm{E}-03$ & $1.38 \mathrm{E}-03$ & 2.86E-03 \\
\hline & 48 & $1.42 \mathrm{E}-04$ & 4.34E-04 & $5.13 \mathrm{E}-05$ & 2.12E-05 & $1.00 \mathrm{E}-05$ & 5.29E-06 & $0.00 E+00$ \\
\hline & 49 & 4.44E-04 & 1.15E-03 & $2.50 \mathrm{E}-04$ & 2.06E-04 & $2.06 \mathrm{E}-04$ & 2.19E-04 & $3.82 \mathrm{E}-04$ \\
\hline & 50 & $4.50 \mathrm{E}-04$ & $1.51 \mathrm{E}-03$ & $1.50 \mathrm{E}-04$ & 5.91E-05 & $2.73 E-05$ & $1.42 \mathrm{E}-05$ & $0.00 E+00$ \\
\hline & 51 & 4.54E-04 & 8.83E-04 & 4.01E-04 & 4.20E-04 & $4.52 \mathrm{E}-04$ & $4.83 \mathrm{E}-04$ & 5.22E-04 \\
\hline & 52 & 4.12E-04 & $1.34 \mathrm{E}-03$ & $1.42 \mathrm{E}-04$ & 5.69E-05 & $2.63 \mathrm{E}-05$ & $1.37 \mathrm{E}-05$ & $0.00 E+00$ \\
\hline & 53 & $1.75 \mathrm{E}-04$ & $6.60 \mathrm{E}-04$ & 5.19E-05 & $1.84 \mathrm{E}-05$ & 7.91E-06 & $4.02 \mathrm{E}-06$ & $0.00 \mathrm{E}+00$ \\
\hline & 54 & $3.22 \mathrm{E}-04$ & 6.37E-04 & $3.09 E-04$ & $3.40 \mathrm{E}-04$ & $3.73 E-04$ & $4.02 \mathrm{E}-04$ & $4.42 \mathrm{E}-04$ \\
\hline & 55 & $1.72 \mathrm{E}-02$ & 9.99E-03 & $2.42 \mathrm{E}-02$ & $3.05 E-02$ & $3.62 \mathrm{E}-02$ & 4.13E-02 & 7.44E-02 \\
\hline & 56 & $1.46 \mathrm{E}-04$ & 4.13E-04 & $5.45 \mathrm{E}-05$ & 2.29E-05 & $1.07 \mathrm{E}-05$ & $5.58 \mathrm{E}-06$ & $0.00 E+00$ \\
\hline & 57 & $1.72 \mathrm{E}-04$ & 4.63E-04 & $6.69 \mathrm{E}-05$ & 2.92E-05 & $1.42 \mathrm{E}-05$ & 7.64E-06 & $0.00 E+00$ \\
\hline & 58 & 4.72E-04 & $6.78 \mathrm{E}-04$ & 4.63E-04 & 5.05E-04 & $5.42 \mathrm{E}-04$ & $5.74 \mathrm{E}-04$ & 5.83E-04 \\
\hline & 59 & 5.23E-05 & $2.20 \mathrm{E}-04$ & $1.46 \mathrm{E}-05$ & 4.91E-06 & $1.90 \mathrm{E}-06$ & $8.21 \mathrm{E}-07$ & $0.00 E+00$ \\
\hline & 60 & 1.59E-04 & $2.41 \mathrm{E}-04$ & $1.73 \mathrm{E}-04$ & $1.96 \mathrm{E}-04$ & $2.13 \mathrm{E}-04$ & $2.25 \mathrm{E}-04$ & 2.19E-04 \\
\hline & 61 & $3.25 \mathrm{E}-04$ & $3.72 \mathrm{E}-04$ & 4.07E-04 & $4.81 \mathrm{E}-04$ & $5.40 \mathrm{E}-04$ & $5.82 \mathrm{E}-04$ & $6.13 \mathrm{E}-04$ \\
\hline & 62 & 7.63E-05 & $3.54 \mathrm{E}-04$ & $2.18 \mathrm{E}-05$ & $7.80 \mathrm{E}-06$ & $3.18 \mathrm{E}-06$ & $1.46 \mathrm{E}-06$ & $0.00 \mathrm{E}+00$ \\
\hline & 63 & 5.97E-05 & $2.73 \mathrm{E}-04$ & $1.55 \mathrm{E}-05$ & $5.15 E-06$ & $2.02 \mathrm{E}-06$ & 8.84E-07 & $0.00 E+00$ \\
\hline & 64 & $1.41 \mathrm{E}-03$ & $5.56 \mathrm{E}-04$ & 2.07E-03 & $2.51 \mathrm{E}-03$ & $2.83 \mathrm{E}-03$ & $3.05 E-03$ & $3.18 \mathrm{E}-03$ \\
\hline & 65 & 3.63E-05 & $1.81 \mathrm{E}-04$ & 8.95E-06 & $3.00 \mathrm{E}-06$ & $1.24 \mathrm{E}-06$ & 5.77E-07 & $0.00 \mathrm{E}+00$ \\
\hline & 66 & $6.78 \mathrm{E}-05$ & $1.71 \mathrm{E}-04$ & $6.09 \mathrm{E}-05$ & $6.70 \mathrm{E}-05$ & 7.27E-05 & $7.66 \mathrm{E}-05$ & 7.48E-05 \\
\hline & 67 & 3.94E-03 & $3.45 \mathrm{E}-03$ & 4.20E-03 & 4.35E-03 & 4.45E-03 & 4.55E-03 & 4.30E-03 \\
\hline
\end{tabular}


Table 4 (continued)

\begin{tabular}{|c|c|c|c|c|c|c|c|c|}
\hline \multirow[b]{2}{*}{$\mathrm{i}$} & \multirow[b]{2}{*}{$\mathrm{j}$} & \multicolumn{7}{|c|}{ collision strengths at energies } \\
\hline & & $65.33 \mathrm{eV}$ & $1714.98 \mathrm{eV}$ & $2944.1 \mathrm{eV}$ & $4868.3 \mathrm{eV}$ & $7564.6 \mathrm{eV}$ & $11040 \mathrm{eV}$ & $15221 \mathrm{eV}$ \\
\hline & 68 & $2.24 \mathrm{E}-04$ & $7.67 \mathrm{E}-04$ & $7.26 \mathrm{E}-05$ & $2.79 \mathrm{E}-05$ & $1.27 \mathrm{E}-05$ & $6.56 \mathrm{E}-06$ & $0.00 E+00$ \\
\hline & 69 & $3.72 \mathrm{E}-04$ & $1.21 \mathrm{E}-03$ & $1.28 \mathrm{E}-04$ & $5.07 E-05$ & $2.34 \mathrm{E}-05$ & $1.22 \mathrm{E}-05$ & $0.00 \mathrm{E}+00$ \\
\hline & 70 & $4.10 \mathrm{E}-04$ & 7.98E-04 & 3.67E-04 & $3.89 \mathrm{E}-04$ & $4.20 \mathrm{E}-04$ & 4.49E-04 & $4.88 \mathrm{E}-04$ \\
\hline & 71 & $1.29 \mathrm{E}-02$ & $7.52 \mathrm{E}-03$ & $1.81 \mathrm{E}-02$ & $2.28 \mathrm{E}-02$ & $2.72 \mathrm{E}-02$ & $3.11 \mathrm{E}-02$ & $5.64 \mathrm{E}-02$ \\
\hline & 72 & $3.96 \mathrm{E}-05$ & $1.88 \mathrm{E}-04$ & $1.08 \mathrm{E}-05$ & $3.83 \mathrm{E}-06$ & $1.58 \mathrm{E}-06$ & $7.29 E-07$ & $0.00 \mathrm{E}+00$ \\
\hline & 73 & $1.08 \mathrm{E}-04$ & $2.08 \mathrm{E}-04$ & 1.07E-04 & 1.19E-04 & $1.30 \mathrm{E}-04$ & $1.37 \mathrm{E}-04$ & $1.33 \mathrm{E}-04$ \\
\hline & 74 & $7.90 \mathrm{E}-04$ & $3.91 \mathrm{E}-04$ & $1.14 \mathrm{E}-03$ & $1.38 \mathrm{E}-03$ & $1.56 \mathrm{E}-03$ & $1.68 \mathrm{E}-03$ & $1.76 \mathrm{E}-03$ \\
\hline & 75 & 7.37E-05 & $3.21 \mathrm{E}-04$ & $1.97 \mathrm{E}-05$ & $6.57 E-06$ & $2.55 \mathrm{E}-06$ & $1.10 \mathrm{E}-06$ & $0.00 E+00$ \\
\hline & 76 & $7.02 \mathrm{E}-05$ & $1.61 \mathrm{E}-04$ & 2.90E-05 & $1.30 \mathrm{E}-05$ & $6.39 \mathrm{E}-06$ & $3.42 \mathrm{E}-06$ & $0.00 E+00$ \\
\hline & 77 & $1.89 \mathrm{E}-04$ & $9.22 \mathrm{E}-05$ & $3.24 \mathrm{E}-04$ & 4.64E-04 & 5.89E-04 & $7.02 \mathrm{E}-04$ & $1.44 \mathrm{E}-03$ \\
\hline & 78 & $1.42 \mathrm{E}-04$ & $3.99 \mathrm{E}-04$ & $5.64 \mathrm{E}-05$ & $2.57 \mathrm{E}-05$ & $1.30 \mathrm{E}-05$ & 7.09E-06 & $0.00 \mathrm{E}+00$ \\
\hline & 79 & $1.74 \mathrm{E}-03$ & $1.43 \mathrm{E}-03$ & $1.91 \mathrm{E}-03$ & 2.01E-03 & $2.08 \mathrm{E}-03$ & $2.13 E-03$ & $2.06 \mathrm{E}-03$ \\
\hline & 80 & $2.26 \mathrm{E}-04$ & $3.25 \mathrm{E}-04$ & $2.20 \mathrm{E}-04$ & 2.37E-04 & $2.55 \mathrm{E}-04$ & 2.69E-04 & 2.71E-04 \\
\hline & 81 & $6.54 \mathrm{E}-05$ & $1.70 \mathrm{E}-04$ & $2.61 \mathrm{E}-05$ & $1.16 \mathrm{E}-05$ & $5.72 \mathrm{E}-06$ & $3.10 \mathrm{E}-06$ & $0.00 E+00$ \\
\hline & 82 & $1.61 \mathrm{E}-04$ & $4.61 \mathrm{E}-04$ & $6.01 \mathrm{E}-05$ & $2.53 \mathrm{E}-05$ & 1.19E-05 & $6.14 \mathrm{E}-06$ & $0.00 \mathrm{E}+00$ \\
\hline & 83 & $6.19 \mathrm{E}-05$ & $1.68 \mathrm{E}-04$ & $2.38 \mathrm{E}-05$ & $1.02 \mathrm{E}-05$ & 4.96E-06 & $2.65 \mathrm{E}-06$ & $0.00 \mathrm{E}+00$ \\
\hline & 84 & $1.86 \mathrm{E}-04$ & $2.55 \mathrm{E}-04$ & $1.89 \mathrm{E}-04$ & 2.09E-04 & $2.27 \mathrm{E}-04$ & $2.41 \mathrm{E}-04$ & 2.45E-04 \\
\hline & 85 & $2.42 \mathrm{E}-03$ & $2.08 \mathrm{E}-03$ & $2.59 \mathrm{E}-03$ & 2.70E-03 & 2.77E-03 & $2.83 \mathrm{E}-03$ & 2.69E-03 \\
\hline & 86 & 4.70E-05 & $1.44 \mathrm{E}-04$ & 1.67E-05 & $6.68 \mathrm{E}-06$ & $2.94 \mathrm{E}-06$ & $1.36 \mathrm{E}-06$ & $0.00 E+00$ \\
\hline & 87 & $2.70 \mathrm{E}-04$ & $3.98 \mathrm{E}-04$ & $3.15 E-04$ & 4.07E-04 & $5.03 \mathrm{E}-04$ & 5.97E-04 & $1.25 \mathrm{E}-03$ \\
\hline & 88 & 4.66E-05 & $1.27 \mathrm{E}-04$ & $1.83 \mathrm{E}-05$ & 8.07E-06 & $4.02 \mathrm{E}-06$ & $2.26 \mathrm{E}-06$ & $0.00 E+00$ \\
\hline & 89 & 6.19E-04 & $5.41 \mathrm{E}-04$ & $8.55 \mathrm{E}-04$ & $1.12 \mathrm{E}-03$ & $1.38 \mathrm{E}-03$ & $1.62 \mathrm{E}-03$ & 3.30E-03 \\
\hline & 90 & 2.07E-04 & $6.82 \mathrm{E}-04$ & 7.02E-05 & $2.74 \mathrm{E}-05$ & $1.23 \mathrm{E}-05$ & $6.08 \mathrm{E}-06$ & $0.00 E+00$ \\
\hline & 91 & $1.78 \mathrm{E}-04$ & $3.89 \mathrm{E}-04$ & 1.49E-04 & $1.55 \mathrm{E}-04$ & $1.67 \mathrm{E}-04$ & $1.81 \mathrm{E}-04$ & $2.00 \mathrm{E}-04$ \\
\hline & 92 & $2.10 \mathrm{E}-04$ & $6.96 \mathrm{E}-04$ & 7.07E-05 & $2.80 \mathrm{E}-05$ & $1.30 \mathrm{E}-05$ & $6.84 \mathrm{E}-06$ & $0.00 \mathrm{E}+00$ \\
\hline & 93 & $7.84 \mathrm{E}-05$ & 2.99E-04 & $2.29 E-05$ & 7.92E-06 & $3.30 \mathrm{E}-06$ & $1.63 \mathrm{E}-06$ & $0.00 E+00$ \\
\hline & 94 & $1.23 \mathrm{E}-04$ & $2.94 \mathrm{E}-04$ & $1.08 \mathrm{E}-04$ & $1.18 \mathrm{E}-04$ & $1.30 \mathrm{E}-04$ & $1.42 \mathrm{E}-04$ & $1.60 \mathrm{E}-04$ \\
\hline & 95 & $1.02 \mathrm{E}-04$ & $2.37 \mathrm{E}-04$ & $4.28 \mathrm{E}-05$ & $1.93 \mathrm{E}-05$ & 9.44E-06 & $5.07 \mathrm{E}-06$ & $0.00 \mathrm{E}+00$ \\
\hline & 96 & $5.70 \mathrm{E}-03$ & $2.65 \mathrm{E}-03$ & 8.87E-03 & $1.18 \mathrm{E}-02$ & $1.46 \mathrm{E}-02$ & $1.70 \mathrm{E}-02$ & $3.38 \mathrm{E}-02$ \\
\hline & 97 & $3.28 \mathrm{E}-03$ & $2.22 \mathrm{E}-03$ & $4.22 \mathrm{E}-03$ & $5.03 \mathrm{E}-03$ & $5.74 \mathrm{E}-03$ & $6.41 \mathrm{E}-03$ & $1.01 \mathrm{E}-02$ \\
\hline & 98 & $5.12 \mathrm{E}-05$ & $1.98 \mathrm{E}-04$ & $1.55 \mathrm{E}-05$ & $5.64 \mathrm{E}-06$ & $2.34 \mathrm{E}-06$ & $1.08 \mathrm{E}-06$ & $0.00 E+00$ \\
\hline & 99 & $2.28 \mathrm{E}-04$ & $3.24 \mathrm{E}-04$ & $2.56 \mathrm{E}-04$ & $2.94 \mathrm{E}-04$ & $3.25 \mathrm{E}-04$ & $3.50 \mathrm{E}-04$ & $3.66 \mathrm{E}-04$ \\
\hline & 100 & 8.69E-05 & 1.49E-04 & $9.22 \mathrm{E}-05$ & $1.04 \mathrm{E}-04$ & $1.15 \mathrm{E}-04$ & $1.22 \mathrm{E}-04$ & 1.20E-04 \\
\hline & 101 & 5.11E-05 & $2.27 \mathrm{E}-04$ & $1.50 \mathrm{E}-05$ & 5.51E-06 & $2.29 \mathrm{E}-06$ & $1.06 \mathrm{E}-06$ & $0.00 \mathrm{E}+00$ \\
\hline & 102 & 5.87E-05 & $2.43 \mathrm{E}-04$ & $1.69 \mathrm{E}-05$ & $6.09 E-06$ & $2.57 \mathrm{E}-06$ & $1.20 \mathrm{E}-06$ & $0.00 \mathrm{E}+00$ \\
\hline & 103 & $6.46 \mathrm{E}-06$ & $3.15 \mathrm{E}-05$ & 1.59E-06 & 4.83E-07 & $1.63 \mathrm{E}-07$ & $6.14 \mathrm{E}-08$ & $0.00 E+00$ \\
\hline & 104 & 4.75E-05 & 4.49E-05 & 5.71E-05 & $6.29 \mathrm{E}-05$ & $6.65 \mathrm{E}-05$ & $6.94 \mathrm{E}-05$ & $6.52 \mathrm{E}-05$ \\
\hline & 105 & $2.11 \mathrm{E}-05$ & $1.07 \mathrm{E}-04$ & $5.20 \mathrm{E}-06$ & $1.77 \mathrm{E}-06$ & $7.74 \mathrm{E}-07$ & $3.80 \mathrm{E}-07$ & $0.00 E+00$ \\
\hline & 106 & $9.40 \mathrm{E}-04$ & 3.79E-04 & $1.37 \mathrm{E}-03$ & $1.67 \mathrm{E}-03$ & $1.88 \mathrm{E}-03$ & $2.03 \mathrm{E}-03$ & 2.12E-03 \\
\hline & 107 & $4.06 \mathrm{E}-05$ & $1.13 \mathrm{E}-04$ & $3.48 \mathrm{E}-05$ & $3.82 \mathrm{E}-05$ & $4.18 \mathrm{E}-05$ & $4.45 \mathrm{E}-05$ & 4.38E-05 \\
\hline & 108 & $6.68 \mathrm{E}-06$ & 1.47E-05 & $6.62 \mathrm{E}-06$ & $7.13 \mathrm{E}-06$ & $7.52 \mathrm{E}-06$ & 7.86E-06 & $7.52 \mathrm{E}-06$ \\
\hline & 109 & $3.76 \mathrm{E}-05$ & $2.33 \mathrm{E}-05$ & $4.82 \mathrm{E}-05$ & 5.37E-05 & $5.69 \mathrm{E}-05$ & 5.95E-05 & 5.57E-05 \\
\hline & 110 & $3.52 \mathrm{E}-06$ & $1.94 \mathrm{E}-05$ & 8.99E-07 & $2.83 \mathrm{E}-07$ & $1.04 \mathrm{E}-07$ & 4.27E-08 & $0.00 E+00$ \\
\hline & 111 & $5.44 \mathrm{E}-06$ & 2.70E-05 & $1.30 \mathrm{E}-06$ & $3.97 E-07$ & $1.39 \mathrm{E}-07$ & $5.42 \mathrm{E}-08$ & $0.00 \mathrm{E}+00$ \\
\hline & 112 & $1.39 \mathrm{E}-06$ & 7.97E-06 & $2.85 \mathrm{E}-07$ & 8.70E-08 & $3.47 \mathrm{E}-08$ & $1.54 \mathrm{E}-08$ & $0.00 E+00$ \\
\hline & 113 & $2.62 \mathrm{E}-06$ & 8.63E-06 & $2.00 \mathrm{E}-06$ & $2.08 \mathrm{E}-06$ & $2.18 \mathrm{E}-06$ & $2.27 \mathrm{E}-06$ & $2.18 \mathrm{E}-06$ \\
\hline & 114 & $1.03 \mathrm{E}-05$ & $2.42 \mathrm{E}-05$ & $4.21 \mathrm{E}-06$ & $1.87 \mathrm{E}-06$ & $9.32 \mathrm{E}-07$ & $5.06 \mathrm{E}-07$ & $0.00 \mathrm{E}+00$ \\
\hline & 115 & $6.41 \mathrm{E}-05$ & $9.00 \mathrm{E}-05$ & $6.14 \mathrm{E}-05$ & $6.66 \mathrm{E}-05$ & 7.31E-05 & 7.93E-05 & $1.00 \mathrm{E}-04$ \\
\hline & 116 & $7.15 E-05$ & $2.13 \mathrm{E}-04$ & $2.56 \mathrm{E}-05$ & $1.05 \mathrm{E}-05$ & 4.84E-06 & $2.43 \mathrm{E}-06$ & $0.00 \mathrm{E}+00$ \\
\hline & 117 & $2.96 \mathrm{E}-04$ & $4.35 \mathrm{E}-04$ & $2.85 \mathrm{E}-04$ & $3.04 \mathrm{E}-04$ & $3.26 \mathrm{E}-04$ & $3.42 \mathrm{E}-04$ & $3.44 \mathrm{E}-04$ \\
\hline & 118 & $8.01 \mathrm{E}-05$ & $2.14 \mathrm{E}-04$ & $3.13 \mathrm{E}-05$ & 1.37E-05 & $6.71 \mathrm{E}-06$ & $3.62 \mathrm{E}-06$ & $0.00 E+00$ \\
\hline & 119 & $1.34 \mathrm{E}-03$ & 1.17E-03 & $1.43 \mathrm{E}-03$ & $1.49 \mathrm{E}-03$ & $1.53 \mathrm{E}-03$ & $1.56 \mathrm{E}-03$ & $1.49 \mathrm{E}-03$ \\
\hline & 120 & 3.09E-04 & 8.33E-04 & $1.20 \mathrm{E}-04$ & $5.24 \mathrm{E}-05$ & $2.58 \mathrm{E}-05$ & $1.40 \mathrm{E}-05$ & $0.00 \mathrm{E}+00$ \\
\hline & 121 & $1.16 \mathrm{E}-04$ & $2.95 \mathrm{E}-04$ & 4.69E-05 & $2.08 \mathrm{E}-05$ & $1.04 \mathrm{E}-05$ & $5.73 \mathrm{E}-06$ & $0.00 \mathrm{E}+00$ \\
\hline & 122 & $6.21 \mathrm{E}-04$ & $7.06 \mathrm{E}-04$ & $6.56 \mathrm{E}-04$ & 7.12E-04 & $7.64 \mathrm{E}-04$ & $8.03 \mathrm{E}-04$ & 7.94E-04 \\
\hline & 123 & $2.81 \mathrm{E}-03$ & $1.61 \mathrm{E}-03$ & $3.73 E-03$ & $4.34 \mathrm{E}-03$ & $4.78 \mathrm{E}-03$ & 5.07E-03 & $5.08 \mathrm{E}-03$ \\
\hline & 124 & $1.03 \mathrm{E}-04$ & $3.51 \mathrm{E}-04$ & $3.38 \mathrm{E}-05$ & $1.30 \mathrm{E}-05$ & 5.97E-06 & $3.12 \mathrm{E}-06$ & $0.00 \mathrm{E}+00$ \\
\hline & 125 & $1.79 \mathrm{E}-04$ & 5.71E-04 & $6.21 \mathrm{E}-05$ & $2.49 \mathrm{E}-05$ & $1.16 \mathrm{E}-05$ & $6.10 \mathrm{E}-06$ & $0.00 E+00$ \\
\hline & 126 & $1.20 \mathrm{E}-04$ & $3.47 \mathrm{E}-04$ & 7.73E-05 & 7.34E-05 & 7.87E-05 & 8.59E-05 & 9.91E-05 \\
\hline & 127 & $3.57 \mathrm{E}-04$ & $2.94 \mathrm{E}-04$ & $4.31 \mathrm{E}-04$ & $4.73 \mathrm{E}-04$ & $5.02 \mathrm{E}-04$ & $5.20 \mathrm{E}-04$ & 4.99E-04 \\
\hline & 128 & 8.49E-05 & 3.34E-04 & $2.53 \mathrm{E}-05$ & $8.86 \mathrm{E}-06$ & 3.44E-06 & $1.43 \mathrm{E}-06$ & $0.00 E+00$ \\
\hline & 129 & $4.28 \mathrm{E}-03$ & $2.57 \mathrm{E}-03$ & $5.95 \mathrm{E}-03$ & $7.46 \mathrm{E}-03$ & 8.84E-03 & $1.01 \mathrm{E}-02$ & $1.81 \mathrm{E}-02$ \\
\hline & 130 & $4.90 \mathrm{E}-05$ & $1.84 \mathrm{E}-04$ & $1.50 \mathrm{E}-05$ & 5.37E-06 & $2.13 \mathrm{E}-06$ & $9.13 \mathrm{E}-07$ & $0.00 E+00$ \\
\hline & 131 & 5.09E-04 & $3.47 \mathrm{E}-04$ & $6.28 \mathrm{E}-04$ & $6.92 \mathrm{E}-04$ & 7.34E-04 & 7.61E-04 & 7.34E-04 \\
\hline & 132 & $2.69 \mathrm{E}-05$ & $1.23 \mathrm{E}-04$ & $7.53 \mathrm{E}-06$ & $2.72 \mathrm{E}-06$ & $1.14 \mathrm{E}-06$ & $5.32 \mathrm{E}-07$ & $0.00 E+00$ \\
\hline & 133 & $6.25 \mathrm{E}-05$ & $1.33 \mathrm{E}-04$ & $5.98 \mathrm{E}-05$ & $6.61 \mathrm{E}-05$ & $7.26 \mathrm{E}-05$ & $7.70 \mathrm{E}-05$ & 7.60E-05 \\
\hline & 134 & $4.05 \mathrm{E}-04$ & $2.08 \mathrm{E}-04$ & $5.87 \mathrm{E}-04$ & $7.18 \mathrm{E}-04$ & 8.09E-04 & $8.81 \mathrm{E}-04$ & $9.23 \mathrm{E}-04$ \\
\hline & 135 & $4.21 \mathrm{E}-05$ & $1.89 \mathrm{E}-04$ & $1.08 \mathrm{E}-05$ & $3.47 \mathrm{E}-06$ & $1.29 \mathrm{E}-06$ & 5.37E-07 & $0.00 \mathrm{E}+00$ \\
\hline
\end{tabular}


Table 4 (continued)

\begin{tabular}{|c|c|c|c|c|c|c|c|c|}
\hline \multirow[b]{2}{*}{$\mathrm{i}$} & \multirow[b]{2}{*}{$\mathrm{j}$} & \multicolumn{7}{|c|}{ collision strengths at energies } \\
\hline & & $65.33 \mathrm{eV}$ & $1714.98 \mathrm{eV}$ & $2944.1 \mathrm{eV}$ & $4868.3 \mathrm{eV}$ & $7564.6 \mathrm{eV}$ & $11040 \mathrm{eV}$ & $15221 \mathrm{eV}$ \\
\hline & 136 & $1.88 \mathrm{E}-06$ & $1.05 \mathrm{E}-05$ & $4.58 \mathrm{E}-07$ & $1.44 \mathrm{E}-07$ & $5.37 \mathrm{E}-08$ & $2.25 \mathrm{E}-08$ & $0.00 E+00$ \\
\hline & 137 & 4.39E-06 & 1.17E-05 & 3.90E-06 & $4.13 \mathrm{E}-06$ & 4.33E-06 & 4.50E-06 & 4.35E-06 \\
\hline & 138 & $1.22 \mathrm{E}-05$ & $1.06 \mathrm{E}-05$ & $1.40 \mathrm{E}-05$ & $1.55 \mathrm{E}-05$ & $1.44 \mathrm{E}-05$ & $1.74 \mathrm{E}-05$ & $1.68 \mathrm{E}-05$ \\
\hline & 139 & 3.47E-06 & $1.06 \mathrm{E}-05$ & 1.09E-06 & 4.16E-07 & $1.82 \mathrm{E}-07$ & $8.45 \mathrm{E}-08$ & $0.00 \mathrm{E}+00$ \\
\hline & 140 & $3.29 E-05$ & $7.55 \mathrm{E}-05$ & $1.35 \mathrm{E}-05$ & $6.04 \mathrm{E}-06$ & 2.99E-06 & $1.61 \mathrm{E}-06$ & $0.00 \mathrm{E}+00$ \\
\hline & 141 & $6.39 \mathrm{E}-05$ & $4.02 \mathrm{E}-05$ & $1.05 \mathrm{E}-04$ & $1.49 \mathrm{E}-04$ & $1.88 \mathrm{E}-04$ & $2.23 \mathrm{E}-04$ & 4.47E-04 \\
\hline & 142 & 5.47E-05 & $1.40 \mathrm{E}-04$ & $2.23 \mathrm{E}-05$ & $1.01 \mathrm{E}-05$ & 5.04E-06 & $2.75 \mathrm{E}-06$ & $0.00 E+00$ \\
\hline & 143 & $9.80 \mathrm{E}-05$ & $1.61 \mathrm{E}-04$ & $8.82 \mathrm{E}-05$ & $9.28 \mathrm{E}-05$ & $9.97 \mathrm{E}-05$ & $1.05 \mathrm{E}-04$ & $1.07 \mathrm{E}-04$ \\
\hline & 144 & $8.71 \mathrm{E}-05$ & 2.47E-04 & $3.29 \mathrm{E}-05$ & 1.40E-05 & $6.60 \mathrm{E}-06$ & $3.44 \mathrm{E}-06$ & $0.00 E+00$ \\
\hline & 145 & $3.66 \mathrm{E}-05$ & $1.02 \mathrm{E}-04$ & 1.39E-05 & $5.88 \mathrm{E}-06$ & $2.82 \mathrm{E}-06$ & $1.49 \mathrm{E}-06$ & $0.00 E+00$ \\
\hline & 146 & $8.00 \mathrm{E}-05$ & $1.27 \mathrm{E}-04$ & $7.58 \mathrm{E}-05$ & $8.23 E-05$ & 8.97E-05 & $9.52 \mathrm{E}-05$ & 9.79E-05 \\
\hline & 147 & 1.17E-03 & $1.00 \mathrm{E}-03$ & $1.26 \mathrm{E}-03$ & $1.31 \mathrm{E}-03$ & $1.35 \mathrm{E}-03$ & $1.38 \mathrm{E}-03$ & $1.31 \mathrm{E}-03$ \\
\hline & 148 & $3.41 \mathrm{E}-05$ & $1.03 E-04$ & $1.25 \mathrm{E}-05$ & $5.20 \mathrm{E}-06$ & $2.47 \mathrm{E}-06$ & $1.31 \mathrm{E}-06$ & $0.00 E+00$ \\
\hline & 149 & $1.02 \mathrm{E}-04$ & $2.66 \mathrm{E}-04$ & $5.62 \mathrm{E}-05$ & $4.52 \mathrm{E}-05$ & $4.46 \mathrm{E}-05$ & 4.73E-05 & 8.23E-05 \\
\hline & 150 & $9.14 \mathrm{E}-05$ & $2.06 \mathrm{E}-04$ & $7.54 \mathrm{E}-05$ & $7.84 \mathrm{E}-05$ & $8.48 \mathrm{E}-05$ & $9.18 \mathrm{E}-05$ & $1.03 \mathrm{E}-04$ \\
\hline & 151 & $1.13 \mathrm{E}-04$ & $3.72 \mathrm{E}-04$ & $3.81 \mathrm{E}-05$ & $1.51 \mathrm{E}-05$ & 7.06E-06 & $3.72 \mathrm{E}-06$ & $0.00 \mathrm{E}+00$ \\
\hline & 152 & 8.97E-05 & $2.93 \mathrm{E}-04$ & $3.08 \mathrm{E}-05$ & $1.23 \mathrm{E}-05$ & $5.67 \mathrm{E}-06$ & 2.97E-06 & $0.00 E+00$ \\
\hline & 153 & $5.12 \mathrm{E}-05$ & $1.85 \mathrm{E}-04$ & $1.59 \mathrm{E}-05$ & $5.80 \mathrm{E}-06$ & $2.53 \mathrm{E}-06$ & $1.29 \mathrm{E}-06$ & $0.00 E+00$ \\
\hline & 154 & $6.37 \mathrm{E}-05$ & 1.59E-04 & 5.47E-05 & $5.96 \mathrm{E}-05$ & $6.60 \mathrm{E}-05$ & $7.22 \mathrm{E}-05$ & $8.22 \mathrm{E}-05$ \\
\hline & 155 & $4.03 E-03$ & $2.34 \mathrm{E}-03$ & 5.61E-03 & 7.03E-03 & $8.30 \mathrm{E}-03$ & $9.47 \mathrm{E}-03$ & $1.68 \mathrm{E}-02$ \\
\hline & 156 & $2.40 \mathrm{E}-05$ & $9.77 \mathrm{E}-05$ & $6.91 \mathrm{E}-06$ & 2.41E-06 & $9.50 \mathrm{E}-07$ & $4.14 \mathrm{E}-07$ & $0.00 E+00$ \\
\hline & 157 & $5.00 \mathrm{E}-05$ & $9.12 \mathrm{E}-05$ & $5.21 \mathrm{E}-05$ & $5.88 \mathrm{E}-05$ & $6.54 \mathrm{E}-05$ & $6.93 \mathrm{E}-05$ & $6.88 \mathrm{E}-05$ \\
\hline & 158 & $9.64 \mathrm{E}-05$ & $1.56 \mathrm{E}-04$ & $1.04 \mathrm{E}-04$ & $1.20 \mathrm{E}-04$ & $1.33 \mathrm{E}-04$ & $1.44 \mathrm{E}-04$ & $1.51 \mathrm{E}-04$ \\
\hline & 159 & $3.24 \mathrm{E}-05$ & $1.41 \mathrm{E}-04$ & $9.62 \mathrm{E}-06$ & $3.58 \mathrm{E}-06$ & $1.51 \mathrm{E}-06$ & 6.99E-07 & $0.00 E+00$ \\
\hline & 160 & $2.56 \mathrm{E}-05$ & $1.15 \mathrm{E}-04$ & 6.87E-06 & $2.34 \mathrm{E}-06$ & $9.55 \mathrm{E}-07$ & $4.36 \mathrm{E}-07$ & $0.00 E+00$ \\
\hline & 161 & $1.64 \mathrm{E}-05$ & $7.96 \mathrm{E}-05$ & $4.15 E-06$ & $1.40 \mathrm{E}-06$ & $5.94 \mathrm{E}-07$ & $2.85 \mathrm{E}-07$ & $0.00 E+00$ \\
\hline & 162 & 4.95E-04 & $2.00 \mathrm{E}-04$ & $7.28 \mathrm{E}-04$ & 8.94E-04 & $1.00 \mathrm{E}-03$ & $1.09 \mathrm{E}-03$ & 1.14E-03 \\
\hline & 163 & $2.45 \mathrm{E}-05$ & $7.25 \mathrm{E}-05$ & 2.04E-05 & $2.23 \mathrm{E}-05$ & 2.47E-05 & $2.61 \mathrm{E}-05$ & $2.60 \mathrm{E}-05$ \\
\hline & 164 & 3.16E-06 & $1.67 \mathrm{E}-05$ & $7.12 \mathrm{E}-07$ & $2.03 \mathrm{E}-07$ & $6.43 \mathrm{E}-08$ & $2.31 \mathrm{E}-08$ & $0.00 \mathrm{E}+00$ \\
\hline & 165 & 5.90E-06 & $1.36 \mathrm{E}-05$ & $5.74 \mathrm{E}-06$ & $6.24 \mathrm{E}-06$ & $6.59 \mathrm{E}-06$ & $6.89 \mathrm{E}-06$ & $6.63 \mathrm{E}-06$ \\
\hline & 166 & $1.33 \mathrm{E}-07$ & $6.68 \mathrm{E}-07$ & $2.11 \mathrm{E}-08$ & $3.94 \mathrm{E}-09$ & $9.98 \mathrm{E}-10$ & $3.85 \mathrm{E}-10$ & $0.00 E+00$ \\
\hline & 167 & $5.74 \mathrm{E}-07$ & 7.61E-07 & $5.92 \mathrm{E}-07$ & 6.47E-07 & $6.98 \mathrm{E}-07$ & 8.68E-07 & $6.84 \mathrm{E}-07$ \\
\hline & 168 & $1.63 \mathrm{E}-07$ & 3.99E-07 & $1.32 \mathrm{E}-07$ & $1.37 \mathrm{E}-07$ & $1.57 \mathrm{E}-07$ & $1.55 \mathrm{E}-07$ & $1.55 \mathrm{E}-07$ \\
\hline & 169 & 3.39E-06 & $1.86 \mathrm{E}-05$ & 8.84E-07 & $2.83 \mathrm{E}-07$ & $1.05 \mathrm{E}-07$ & 4.34E-08 & $0.00 E+00$ \\
\hline & 170 & $1.74 \mathrm{E}-05$ & $2.21 \mathrm{E}-05$ & $2.02 \mathrm{E}-05$ & $2.23 \mathrm{E}-05$ & $2.36 \mathrm{E}-05$ & $2.43 \mathrm{E}-05$ & 2.32E-05 \\
\hline & 171 & $9.38 \mathrm{E}-08$ & 5.47E-07 & $1.54 \mathrm{E}-08$ & 3.67E-09 & $1.08 \mathrm{E}-09$ & $4.31 \mathrm{E}-10$ & $0.00 E+00$ \\
\hline & 172 & $3.89 \mathrm{E}-07$ & $2.42 \mathrm{E}-07$ & $4.40 \mathrm{E}-07$ & $4.88 \mathrm{E}-07$ & $5.28 \mathrm{E}-07$ & $6.44 \mathrm{E}-07$ & 5.15E-07 \\
\hline & 173 & $6.02 \mathrm{E}-08$ & $1.86 \mathrm{E}-07$ & $9.29 \mathrm{E}-09$ & 2.19E-09 & $6.47 \mathrm{E}-10$ & $2.37 \mathrm{E}-10$ & $0.00 E+00$ \\
\hline & 174 & $1.98 \mathrm{E}-05$ & $1.22 \mathrm{E}-05$ & $2.61 \mathrm{E}-05$ & $2.94 \mathrm{E}-05$ & $3.12 \mathrm{E}-05$ & $3.22 \mathrm{E}-05$ & $3.06 \mathrm{E}-05$ \\
\hline & 175 & $7.52 \mathrm{E}-08$ & $2.59 \mathrm{E}-07$ & 1.17E-08 & $2.58 \mathrm{E}-09$ & $7.32 \mathrm{E}-10$ & $2.70 \mathrm{E}-10$ & $0.00 E+00$ \\
\hline & 176 & 1.59E-06 & $9.41 \mathrm{E}-06$ & 3.31E-07 & $9.86 \mathrm{E}-08$ & $3.78 \mathrm{E}-08$ & $1.64 \mathrm{E}-08$ & $0.00 \mathrm{E}+00$ \\
\hline & 177 & $6.76 \mathrm{E}-08$ & $5.64 \mathrm{E}-08$ & 4.17E-08 & 4.10E-08 & 4.66E-08 & 4.56E-08 & 4.59E-08 \\
\hline & 178 & $2.56 \mathrm{E}-06$ & $1.43 \mathrm{E}-05$ & $5.42 \mathrm{E}-07$ & $1.56 \mathrm{E}-07$ & $5.41 \mathrm{E}-08$ & $2.16 \mathrm{E}-08$ & $0.00 E+00$ \\
\hline & 179 & $2.41 \mathrm{E}-06$ & $8.76 \mathrm{E}-06$ & $1.76 \mathrm{E}-06$ & $1.84 \mathrm{E}-06$ & $1.92 \mathrm{E}-06$ & $2.01 \mathrm{E}-06$ & $1.94 \mathrm{E}-06$ \\
\hline & 180 & $6.77 \mathrm{E}-06$ & $1.56 \mathrm{E}-05$ & $2.80 \mathrm{E}-06$ & $1.26 \mathrm{E}-06$ & $6.25 \mathrm{E}-07$ & 3.39E-07 & $0.00 E+00$ \\
\hline & 181 & $3.74 \mathrm{E}-05$ & 4.59E-05 & $4.56 \mathrm{E}-05$ & 5.91E-05 & $7.24 \mathrm{E}-05$ & $8.45 E-05$ & $1.63 \mathrm{E}-04$ \\
\hline & 182 & $3.70 \mathrm{E}-05$ & $1.04 \mathrm{E}-04$ & $1.39 \mathrm{E}-05$ & 5.87E-06 & $2.78 \mathrm{E}-06$ & $1.45 \mathrm{E}-06$ & $0.00 E+00$ \\
\hline & 183 & 4.43E-05 & $1.18 \mathrm{E}-04$ & $1.74 \mathrm{E}-05$ & 7.63E-06 & $3.74 \mathrm{E}-06$ & $2.02 \mathrm{E}-06$ & $0.00 \mathrm{E}+00$ \\
\hline & 184 & $9.29 \mathrm{E}-05$ & $1.56 \mathrm{E}-04$ & $8.22 \mathrm{E}-05$ & $8.62 \mathrm{E}-05$ & $9.25 \mathrm{E}-05$ & $9.76 \mathrm{E}-05$ & $1.01 \mathrm{E}-04$ \\
\hline & 185 & $6.42 \mathrm{E}-04$ & 5.57E-04 & $6.88 \mathrm{E}-04$ & $7.16 \mathrm{E}-04$ & $7.35 \mathrm{E}-04$ & $7.51 \mathrm{E}-04$ & 7.19E-04 \\
\hline & 186 & $5.76 \mathrm{E}-05$ & $1.94 \mathrm{E}-04$ & $1.90 \mathrm{E}-05$ & 7.36E-06 & 3.37E-06 & $1.76 \mathrm{E}-06$ & $0.00 E+00$ \\
\hline & 187 & $9.67 \mathrm{E}-05$ & $3.08 \mathrm{E}-04$ & 3.37E-05 & $1.35 \mathrm{E}-05$ & $6.29 \mathrm{E}-06$ & $3.31 \mathrm{E}-06$ & $0.00 E+00$ \\
\hline & 188 & 8.33E-05 & $1.95 \mathrm{E}-04$ & $6.68 \mathrm{E}-05$ & $6.89 \mathrm{E}-05$ & $7.45 \mathrm{E}-05$ & $8.06 \mathrm{E}-05$ & $9.04 \mathrm{E}-05$ \\
\hline & 189 & $2.13 \mathrm{E}-03$ & $1.29 \mathrm{E}-03$ & $2.96 \mathrm{E}-03$ & $3.71 \mathrm{E}-03$ & $4.38 \mathrm{E}-03$ & $5.00 \mathrm{E}-03$ & $8.92 \mathrm{E}-03$ \\
\hline & 190 & $1.73 \mathrm{E}-05$ & $7.83 \mathrm{E}-05$ & 4.91E-06 & 1.79E-06 & 7.57E-07 & $3.56 \mathrm{E}-07$ & $0.00 E+00$ \\
\hline & 191 & $3.41 \mathrm{E}-05$ & $1.43 \mathrm{E}-04$ & $9.53 E-06$ & $3.29 \mathrm{E}-06$ & $1.33 \mathrm{E}-06$ & 5.99E-07 & $0.00 E+00$ \\
\hline & 192 & 3.69E-05 & $8.35 \mathrm{E}-05$ & $3.45 \mathrm{E}-05$ & $3.80 \mathrm{E}-05$ & $4.20 \mathrm{E}-05$ & 4.47E-05 & 4.43E-05 \\
\hline & 193 & $2.73 \mathrm{E}-04$ & $1.50 \mathrm{E}-04$ & $3.91 \mathrm{E}-04$ & 4.79E-04 & $5.38 \mathrm{E}-04$ & $5.86 \mathrm{E}-04$ & $6.13 \mathrm{E}-04$ \\
\hline & 194 & $1.84 \mathrm{E}-06$ & $1.04 \mathrm{E}-05$ & $4.55 \mathrm{E}-07$ & $1.44 \mathrm{E}-07$ & $5.44 \mathrm{E}-08$ & $2.30 \mathrm{E}-08$ & $0.00 E+00$ \\
\hline & 195 & $4.08 \mathrm{E}-07$ & 4.04E-07 & $4.48 \mathrm{E}-07$ & $4.96 \mathrm{E}-07$ & $5.25 \mathrm{E}-07$ & 5.67E-07 & $5.25 \mathrm{E}-07$ \\
\hline & 196 & $1.78 \mathrm{E}-05$ & 1.49E-05 & $2.26 \mathrm{E}-05$ & $2.54 \mathrm{E}-05$ & 2.69E-05 & $2.76 \mathrm{E}-05$ & $2.64 \mathrm{E}-05$ \\
\hline & 197 & $5.41 \mathrm{E}-08$ & 2.70E-07 & 8.67E-09 & $2.13 \mathrm{E}-09$ & $6.37 \mathrm{E}-10$ & $2.47 \mathrm{E}-10$ & $0.00 E+00$ \\
\hline & 198 & $1.27 \mathrm{E}-07$ & $5.93 \mathrm{E}-07$ & $2.02 \mathrm{E}-08$ & $4.02 \mathrm{E}-09$ & $1.05 \mathrm{E}-09$ & $3.97 \mathrm{E}-10$ & $0.00 \mathrm{E}+00$ \\
\hline & 199 & $3.96 \mathrm{E}-06$ & 1.13E-05 & 3.43E-06 & 3.66E-06 & 3.83E-06 & $4.00 \mathrm{E}-06$ & 3.87E-06 \\
\hline & 200 & $1.06 \mathrm{E}-07$ & $2.65 \mathrm{E}-07$ & 7.84E-08 & $7.95 \mathrm{E}-08$ & $8.64 \mathrm{E}-08$ & $9.54 \mathrm{E}-08$ & 8.83E-08 \\
\hline & 201 & $3.71 \mathrm{E}-06$ & $1.98 \mathrm{E}-05$ & $8.24 \mathrm{E}-07$ & $2.38 \mathrm{E}-07$ & $7.86 \mathrm{E}-08$ & $2.96 \mathrm{E}-08$ & $0.00 \mathrm{E}+00$ \\
\hline & 202 & 4.06E-05 & $1.21 \mathrm{E}-04$ & $1.59 \mathrm{E}-05$ & $7.24 \mathrm{E}-06$ & $3.69 \mathrm{E}-06$ & $2.02 \mathrm{E}-06$ & $0.00 \mathrm{E}+00$ \\
\hline & 203 & $8.85 \mathrm{E}-04$ & $7.20 \mathrm{E}-04$ & $9.74 \mathrm{E}-04$ & $1.02 \mathrm{E}-03$ & $1.06 \mathrm{E}-03$ & $1.08 \mathrm{E}-03$ & $1.05 \mathrm{E}-03$ \\
\hline
\end{tabular}


Table 4 (continued)

\begin{tabular}{|c|c|c|c|c|c|c|c|c|}
\hline \multirow[b]{2}{*}{$\mathrm{i}$} & \multirow[b]{2}{*}{ j } & \multicolumn{7}{|c|}{ collision strengths at energies } \\
\hline & & $65.33 \mathrm{eV}$ & $1714.98 \mathrm{eV}$ & $2944.1 \mathrm{eV}$ & $4868.3 \mathrm{eV}$ & $7564.6 \mathrm{eV}$ & $11040 \mathrm{eV}$ & $15221 \mathrm{eV}$ \\
\hline & 204 & $1.26 \mathrm{E}-05$ & $3.27 \mathrm{E}-05$ & $4.98 \mathrm{E}-06$ & $2.14 \mathrm{E}-06$ & $9.99 \mathrm{E}-07$ & $4.95 \mathrm{E}-07$ & $0.00 E+00$ \\
\hline & 205 & $2.15 \mathrm{E}-04$ & $1.44 \mathrm{E}-04$ & $3.33 \mathrm{E}-04$ & 4.57E-04 & $5.76 \mathrm{E}-04$ & $6.82 \mathrm{E}-04$ & $1.41 \mathrm{E}-03$ \\
\hline & 206 & $6.05 \mathrm{E}-05$ & $1.55 \mathrm{E}-04$ & 2.41E-05 & $1.04 \mathrm{E}-05$ & $4.88 \mathrm{E}-06$ & $2.43 \mathrm{E}-06$ & $0.00 \mathrm{E}+00$ \\
\hline & 207 & $6.18 \mathrm{E}-04$ & $2.33 \mathrm{E}-04$ & $1.08 \mathrm{E}-03$ & $1.53 \mathrm{E}-03$ & $1.94 \mathrm{E}-03$ & 2.31E-03 & $4.88 \mathrm{E}-03$ \\
\hline & 208 & $6.00 \mathrm{E}-05$ & $1.58 \mathrm{E}-04$ & $2.37 \mathrm{E}-05$ & $1.04 \mathrm{E}-05$ & 5.14E-06 & $2.81 \mathrm{E}-06$ & $0.00 \mathrm{E}+00$ \\
\hline & 209 & $1.22 \mathrm{E}-04$ & $2.71 \mathrm{E}-04$ & $7.02 E-05$ & 5.39E-05 & 4.90E-05 & $4.80 \mathrm{E}-05$ & $4.28 \mathrm{E}-05$ \\
\hline & 210 & $1.39 \mathrm{E}-04$ & $3.66 \mathrm{E}-04$ & $5.47 \mathrm{E}-05$ & $2.40 \mathrm{E}-05$ & 1.19E-05 & $6.48 \mathrm{E}-06$ & $0.00 E+00$ \\
\hline & 211 & $1.21 \mathrm{E}-03$ & $6.99 \mathrm{E}-04$ & $1.61 \mathrm{E}-03$ & $1.87 \mathrm{E}-03$ & 2.07E-03 & $2.20 \mathrm{E}-03$ & $2.20 \mathrm{E}-03$ \\
\hline & 212 & $3.15 \mathrm{E}-05$ & 1.17E-04 & $9.78 \mathrm{E}-06$ & $3.55 \mathrm{E}-06$ & $1.43 \mathrm{E}-06$ & $6.13 \mathrm{E}-07$ & $0.00 E+00$ \\
\hline & 213 & $7.21 \mathrm{E}-05$ & $1.66 \mathrm{E}-04$ & 5.33E-05 & $5.05 E-05$ & 5.07E-05 & $5.08 \mathrm{E}-05$ & 4.86E-05 \\
\hline & 214 & $5.62 \mathrm{E}-05$ & 2.09E-04 & $1.75 \mathrm{E}-05$ & $6.34 \mathrm{E}-06$ & $2.55 \mathrm{E}-06$ & $1.10 \mathrm{E}-06$ & $0.00 \mathrm{E}+00$ \\
\hline & 215 & $3.86 \mathrm{E}-04$ & 2.01E-04 & $5.02 \mathrm{E}-04$ & $5.64 \mathrm{E}-04$ & $6.00 \mathrm{E}-04$ & $6.14 \mathrm{E}-04$ & $6.03 \mathrm{E}-04$ \\
\hline & 216 & $9.33 \mathrm{E}-06$ & $1.51 \mathrm{E}-05$ & $9.63 \mathrm{E}-06$ & $1.05 \mathrm{E}-05$ & 1.14E-05 & $1.31 \mathrm{E}-05$ & 1.16E-05 \\
\hline & 217 & $2.37 \mathrm{E}-06$ & $1.31 \mathrm{E}-05$ & 5.41E-07 & $1.63 \mathrm{E}-07$ & 5.77E-08 & $2.42 \mathrm{E}-08$ & $0.00 E+00$ \\
\hline & 218 & $3.72 \mathrm{E}-06$ & $2.05 \mathrm{E}-05$ & 8.47E-07 & $2.56 \mathrm{E}-07$ & $9.05 \mathrm{E}-08$ & $3.80 \mathrm{E}-08$ & $0.00 E+00$ \\
\hline & 219 & $1.23 \mathrm{E}-05$ & $1.42 \mathrm{E}-05$ & 1.39E-05 & $1.55 \mathrm{E}-05$ & $1.68 \mathrm{E}-05$ & $1.93 \mathrm{E}-05$ & $1.72 \mathrm{E}-05$ \\
\hline & 220 & $2.38 \mathrm{E}-05$ & 7.05E-05 & $9.29 E-06$ & $4.23 \mathrm{E}-06$ & 2.16E-06 & $1.18 \mathrm{E}-06$ & $0.00 E+00$ \\
\hline & 221 & 4.37E-04 & $3.52 \mathrm{E}-04$ & $4.82 \mathrm{E}-04$ & 5.07E-04 & $5.23 \mathrm{E}-04$ & $5.36 \mathrm{E}-04$ & 5.19E-04 \\
\hline & 222 & 7.14E-06 & $1.86 \mathrm{E}-05$ & $2.83 \mathrm{E}-06$ & $1.21 \mathrm{E}-06$ & $5.67 \mathrm{E}-07$ & $2.81 \mathrm{E}-07$ & $0.00 \mathrm{E}+00$ \\
\hline & 223 & 1.17E-04 & 8.05E-05 & 1.79E-04 & $2.45 \mathrm{E}-04$ & 3.09E-04 & 3.67E-04 & $7.56 \mathrm{E}-04$ \\
\hline & 224 & $3.42 \mathrm{E}-05$ & 8.77E-05 & $1.36 \mathrm{E}-05$ & $5.88 \mathrm{E}-06$ & $2.75 \mathrm{E}-06$ & $1.37 \mathrm{E}-06$ & $0.00 E+00$ \\
\hline & 225 & $3.30 \mathrm{E}-04$ & $1.25 \mathrm{E}-04$ & $5.72 \mathrm{E}-04$ & 8.10E-04 & $1.03 \mathrm{E}-03$ & $1.23 \mathrm{E}-03$ & $2.58 \mathrm{E}-03$ \\
\hline & 226 & $3.20 \mathrm{E}-05$ & $8.36 \mathrm{E}-05$ & $1.27 \mathrm{E}-05$ & 5.57E-06 & $2.76 \mathrm{E}-06$ & $1.51 \mathrm{E}-06$ & $0.00 E+00$ \\
\hline & 227 & $6.55 \mathrm{E}-05$ & $1.44 \mathrm{E}-04$ & $3.85 \mathrm{E}-05$ & $3.02 \mathrm{E}-05$ & $2.77 \mathrm{E}-05$ & $2.73 \mathrm{E}-05$ & $2.46 \mathrm{E}-05$ \\
\hline & 228 & $7.40 \mathrm{E}-05$ & $1.94 \mathrm{E}-04$ & $2.93 \mathrm{E}-05$ & $1.29 \mathrm{E}-05$ & $6.38 \mathrm{E}-06$ & $3.49 \mathrm{E}-06$ & $0.00 E+00$ \\
\hline & 229 & 5.81E-04 & 3.39E-04 & $7.74 \mathrm{E}-04$ & $9.04 \mathrm{E}-04$ & 9.99E-04 & $1.07 \mathrm{E}-03$ & $1.07 \mathrm{E}-03$ \\
\hline & 230 & $2.00 \mathrm{E}-05$ & $7.29 \mathrm{E}-05$ & $6.29 \mathrm{E}-06$ & $2.31 \mathrm{E}-06$ & $9.42 \mathrm{E}-07$ & 4.09E-07 & $0.00 E+00$ \\
\hline & 231 & $4.13 \mathrm{E}-05$ & $1.03 \mathrm{E}-04$ & 2.77E-05 & $2.51 \mathrm{E}-05$ & $2.48 \mathrm{E}-05$ & 2.49E-05 & $2.35 \mathrm{E}-05$ \\
\hline & 232 & $3.58 \mathrm{E}-05$ & $1.31 \mathrm{E}-04$ & 1.13E-05 & 4.13E-06 & 1.69E-06 & 7.31E-07 & $0.00 \mathrm{E}+00$ \\
\hline & 233 & $2.25 \mathrm{E}-04$ & $1.16 \mathrm{E}-04$ & $2.96 \mathrm{E}-04$ & $3.34 \mathrm{E}-04$ & $3.56 \mathrm{E}-04$ & $3.68 \mathrm{E}-04$ & 3.59E-04 \\
\hline & 234 & $2.31 \mathrm{E}-06$ & $1.28 \mathrm{E}-05$ & $5.38 \mathrm{E}-07$ & $1.65 \mathrm{E}-07$ & 5.91E-08 & $2.49 \mathrm{E}-08$ & $0.00 E+00$ \\
\hline & 235 & 7.95E-06 & $1.48 \mathrm{E}-05$ & $7.92 \mathrm{E}-06$ & 8.70E-06 & $9.40 \mathrm{E}-06$ & 9.93E-06 & $9.59 \mathrm{E}-06$ \\
\hline & 236 & $2.23 \mathrm{E}-07$ & $7.54 \mathrm{E}-07$ & $2.26 \mathrm{E}-07$ & $6.75 \mathrm{E}-07$ & $2.83 \mathrm{E}-07$ & $3.00 \mathrm{E}-07$ & $2.35 \mathrm{E}-07$ \\
\hline & 237 & $6.52 \mathrm{E}-08$ & $6.59 \mathrm{E}-07$ & $1.21 \mathrm{E}-08$ & $2.88 \mathrm{E}-09$ & $9.29 \mathrm{E}-10$ & $3.51 \mathrm{E}-10$ & $0.00 E+00$ \\
\hline & 238 & $3.63 \mathrm{E}-06$ & $2.00 \mathrm{E}-05$ & 8.44E-07 & 2.59E-07 & $9.27 \mathrm{E}-08$ & 3.89E-08 & $0.00 E+00$ \\
\hline & 239 & $9.41 \mathrm{E}-08$ & $9.50 \mathrm{E}-07$ & $1.75 \mathrm{E}-08$ & 4.16E-09 & $1.34 \mathrm{E}-09$ & $5.07 \mathrm{E}-10$ & $0.00 \mathrm{E}+00$ \\
\hline & 240 & $2.51 \mathrm{E}-07$ & $7.38 \mathrm{E}-07$ & $2.68 \mathrm{E}-07$ & 8.13E-07 & 3.39E-07 & 3.60E-07 & 2.83E-07 \\
\hline & 241 & 1.14E-05 & $1.35 \mathrm{E}-05$ & $1.30 \mathrm{E}-05$ & $1.46 \mathrm{E}-05$ & $1.59 \mathrm{E}-05$ & $1.68 \mathrm{E}-05$ & $1.64 \mathrm{E}-05$ \\
\hline \multirow[t]{30}{*}{2} & 3 & 1.07E-02 & $2.78 \mathrm{E}-02$ & $1.12 \mathrm{E}-02$ & 8.00E-03 & $6.46 \mathrm{E}-03$ & $5.78 \mathrm{E}-03$ & $5.10 \mathrm{E}-03$ \\
\hline & 4 & $5.43 \mathrm{E}-03$ & 7.39E-03 & $5.47 \mathrm{E}-03$ & 5.21E-03 & $5.21 \mathrm{E}-03$ & 5.32E-03 & $5.42 \mathrm{E}-03$ \\
\hline & 5 & $1.32 \mathrm{E}-02$ & $2.54 \mathrm{E}-02$ & $1.36 \mathrm{E}-02$ & $1.06 \mathrm{E}-02$ & $9.00 \mathrm{E}-03$ & $8.30 \mathrm{E}-03$ & $7.32 \mathrm{E}-03$ \\
\hline & 6 & $1.63 \mathrm{E}+00$ & $9.63 \mathrm{E}-01$ & $1.60 \mathrm{E}+00$ & $1.83 \mathrm{E}+00$ & $2.01 \mathrm{E}+00$ & $2.16 \mathrm{E}+00$ & $2.85 \mathrm{E}+00$ \\
\hline & 7 & $1.39 \mathrm{E}+00$ & $8.32 \mathrm{E}-01$ & $1.37 \mathrm{E}+00$ & $1.56 \mathrm{E}+00$ & $1.72 \mathrm{E}+00$ & $1.85 \mathrm{E}+00$ & $2.46 \mathrm{E}+00$ \\
\hline & 8 & $3.87 E+00$ & $2.32 \mathrm{E}+00$ & $3.81 \mathrm{E}+00$ & $4.34 \mathrm{E}+00$ & $4.79 E+00$ & $5.18 \mathrm{E}+00$ & $6.96 \mathrm{E}+00$ \\
\hline & 9 & $3.60 \mathrm{E}-03$ & $7.35 \mathrm{E}-03$ & $3.63 \mathrm{E}-03$ & $3.49 \mathrm{E}-03$ & $3.58 \mathrm{E}-03$ & $3.75 \mathrm{E}-03$ & $4.90 \mathrm{E}-03$ \\
\hline & 10 & $1.45 \mathrm{E}+00$ & $8.82 \mathrm{E}-01$ & $1.43 \mathrm{E}+00$ & $1.63 \mathrm{E}+00$ & $1.80 \mathrm{E}+00$ & $1.95 \mathrm{E}+00$ & $2.64 \mathrm{E}+00$ \\
\hline & 11 & $1.91 \mathrm{E}-04$ & $1.33 \mathrm{E}-03$ & $2.09 \mathrm{E}-04$ & $8.92 \mathrm{E}-05$ & $3.56 \mathrm{E}-05$ & $1.26 \mathrm{E}-05$ & $0.00 \mathrm{E}+00$ \\
\hline & 12 & $2.04 \mathrm{E}-03$ & $1.29 \mathrm{E}-03$ & $2.01 \mathrm{E}-03$ & 2.29E-03 & $2.55 \mathrm{E}-03$ & 2.77E-03 & $3.84 \mathrm{E}-03$ \\
\hline & 13 & $3.65 \mathrm{E}-02$ & $2.28 \mathrm{E}-02$ & $3.59 \mathrm{E}-02$ & 4.12E-02 & 4.59E-02 & $5.00 \mathrm{E}-02$ & 6.97E-02 \\
\hline & 14 & $5.14 \mathrm{E}-03$ & $3.22 \mathrm{E}-03$ & $5.06 \mathrm{E}-03$ & $5.80 \mathrm{E}-03$ & $6.46 \mathrm{E}-03$ & 7.05E-03 & $9.84 \mathrm{E}-03$ \\
\hline & 15 & $7.95 \mathrm{E}-05$ & $5.08 \mathrm{E}-04$ & 8.67E-05 & 3.79E-05 & $1.57 \mathrm{E}-05$ & $6.08 \mathrm{E}-06$ & $0.00 E+00$ \\
\hline & 16 & $1.82 \mathrm{E}-02$ & $1.62 \mathrm{E}-02$ & $1.82 \mathrm{E}-02$ & $1.86 \mathrm{E}-02$ & $1.90 \mathrm{E}-02$ & $1.92 \mathrm{E}-02$ & $1.86 \mathrm{E}-02$ \\
\hline & 17 & $5.27 \mathrm{E}-02$ & 4.69E-02 & $5.25 \mathrm{E}-02$ & $5.38 \mathrm{E}-02$ & $5.48 \mathrm{E}-02$ & 5.55E-02 & $5.36 \mathrm{E}-02$ \\
\hline & 18 & $1.60 \mathrm{E}-01$ & $1.41 \mathrm{E}-01$ & $1.59 \mathrm{E}-01$ & $1.63 \mathrm{E}-01$ & $1.66 \mathrm{E}-01$ & $1.68 \mathrm{E}-01$ & $1.63 \mathrm{E}-01$ \\
\hline & 19 & 7.49E-02 & $6.73 \mathrm{E}-02$ & 7.47E-02 & $7.65 \mathrm{E}-02$ & $7.78 \mathrm{E}-02$ & $7.88 \mathrm{E}-02$ & $7.61 \mathrm{E}-02$ \\
\hline & 20 & $5.56 \mathrm{E}-02$ & $5.46 \mathrm{E}-02$ & $5.55 \mathrm{E}-02$ & 5.63E-02 & 5.71E-02 & 5.77E-02 & 5.57E-02 \\
\hline & 21 & $1.39 \mathrm{E}-02$ & 1.79E-02 & $1.39 \mathrm{E}-02$ & $1.36 \mathrm{E}-02$ & $1.36 \mathrm{E}-02$ & 1.37E-02 & $1.32 \mathrm{E}-02$ \\
\hline & 22 & $6.85 \mathrm{E}-02$ & $6.45 \mathrm{E}-02$ & $6.84 \mathrm{E}-02$ & 6.97E-02 & $7.08 \mathrm{E}-02$ & 7.17E-02 & $6.92 \mathrm{E}-02$ \\
\hline & 23 & $1.93 \mathrm{E}-03$ & $5.35 \mathrm{E}-03$ & 1.99E-03 & $1.61 \mathrm{E}-03$ & $1.47 \mathrm{E}-03$ & $1.42 \mathrm{E}-03$ & $1.33 \mathrm{E}-03$ \\
\hline & 24 & 5.16E-05 & $8.02 \mathrm{E}-05$ & $5.20 \mathrm{E}-05$ & $4.92 \mathrm{E}-05$ & 4.87E-05 & $4.88 \mathrm{E}-05$ & 4.65E-05 \\
\hline & 25 & $1.14 \mathrm{E}-03$ & $9.99 \mathrm{E}-04$ & $1.13 \mathrm{E}-03$ & 1.17E-03 & 1.19E-03 & $1.21 \mathrm{E}-03$ & 1.17E-03 \\
\hline & 26 & 5.01E-04 & 4.35E-04 & 4.99E-04 & 5.15E-04 & $5.26 \mathrm{E}-04$ & 5.34E-04 & $5.15 \mathrm{E}-04$ \\
\hline & 27 & $2.41 \mathrm{E}-04$ & $6.28 \mathrm{E}-04$ & $2.48 \mathrm{E}-04$ & $2.04 \mathrm{E}-04$ & $1.87 \mathrm{E}-04$ & $1.81 \mathrm{E}-04$ & $1.68 \mathrm{E}-04$ \\
\hline & 28 & $3.71 \mathrm{E}-01$ & $2.60 \mathrm{E}-01$ & $3.65 \mathrm{E}-01$ & $4.18 \mathrm{E}-01$ & $4.68 \mathrm{E}-01$ & 5.14E-01 & 7.47E-01 \\
\hline & 29 & $1.13 \mathrm{E}-03$ & $2.45 \mathrm{E}-03$ & 1.19E-03 & 7.17E-04 & 4.17E-04 & $2.44 \mathrm{E}-04$ & $0.00 E+00$ \\
\hline & 30 & 7.04E-06 & $1.03 \mathrm{E}-05$ & $7.06 \mathrm{E}-06$ & 7.10E-06 & $7.50 \mathrm{E}-06$ & 7.97E-06 & 8.70E-06 \\
\hline & 31 & $1.40 \mathrm{E}-05$ & 2.47E-05 & $1.42 \mathrm{E}-05$ & $1.32 \mathrm{E}-05$ & $1.33 \mathrm{E}-05$ & 1.37E-05 & $1.46 \mathrm{E}-05$ \\
\hline & 32 & $1.83 \mathrm{E}-04$ & $2.18 \mathrm{E}-04$ & $1.84 \mathrm{E}-04$ & $1.75 \mathrm{E}-04$ & $1.71 \mathrm{E}-04$ & $1.68 \mathrm{E}-04$ & $1.83 \mathrm{E}-04$ \\
\hline
\end{tabular}


Table 4 (continued)

\begin{tabular}{|c|c|c|c|c|c|c|c|c|}
\hline \multirow[b]{2}{*}{$\mathrm{i}$} & \multirow[b]{2}{*}{$\mathrm{j}$} & \multicolumn{7}{|c|}{ collision strengths at energies } \\
\hline & & $65.33 \mathrm{eV}$ & $1714.98 \mathrm{eV}$ & $2944.1 \mathrm{eV}$ & $4868.3 \mathrm{eV}$ & $7564.6 \mathrm{eV}$ & $11040 \mathrm{eV}$ & $15221 \mathrm{eV}$ \\
\hline & 33 & $7.82 \mathrm{E}-06$ & $1.89 \mathrm{E}-05$ & $8.07 E-06$ & $6.34 \mathrm{E}-06$ & $5.59 \mathrm{E}-06$ & $5.32 \mathrm{E}-06$ & $4.90 \mathrm{E}-06$ \\
\hline & 34 & 7.11E-05 & $6.79 \mathrm{E}-05$ & 7.07E-05 & $7.51 \mathrm{E}-05$ & $7.52 \mathrm{E}-05$ & $8.20 \mathrm{E}-05$ & 8.74E-05 \\
\hline & 35 & $8.14 \mathrm{E}-05$ & $8.31 \mathrm{E}-05$ & $8.10 \mathrm{E}-05$ & $8.56 \mathrm{E}-05$ & 8.69E-05 & $9.34 \mathrm{E}-05$ & $1.00 \mathrm{E}-04$ \\
\hline & 36 & $6.95 \mathrm{E}-05$ & $8.06 \mathrm{E}-05$ & $6.92 \mathrm{E}-05$ & $7.21 \mathrm{E}-05$ & $7.35 \mathrm{E}-05$ & $7.80 \mathrm{E}-05$ & $8.26 \mathrm{E}-05$ \\
\hline & 37 & $5.36 \mathrm{E}-06$ & $3.02 \mathrm{E}-05$ & 5.77E-06 & $3.11 \mathrm{E}-06$ & $2.10 \mathrm{E}-06$ & $1.72 \mathrm{E}-06$ & $1.76 \mathrm{E}-06$ \\
\hline & 38 & $1.52 \mathrm{E}-01$ & $1.31 \mathrm{E}-01$ & $1.51 \mathrm{E}-01$ & $1.55 \mathrm{E}-01$ & $1.56 \mathrm{E}-01$ & $1.59 \mathrm{E}-01$ & $1.55 \mathrm{E}-01$ \\
\hline & 39 & 2.45E-04 & $1.72 \mathrm{E}-03$ & $2.70 \mathrm{E}-04$ & $1.06 \mathrm{E}-04$ & 4.60E-05 & 2.42E-05 & $2.49 \mathrm{E}-06$ \\
\hline & 40 & $2.70 \mathrm{E}-02$ & $5.48 \mathrm{E}-03$ & $2.58 \mathrm{E}-02$ & $3.82 \mathrm{E}-02$ & $5.04 \mathrm{E}-02$ & $6.22 \mathrm{E}-02$ & $1.41 \mathrm{E}-01$ \\
\hline & 41 & $2.19 \mathrm{E}-02$ & $5.56 \mathrm{E}-03$ & 2.09E-02 & $3.08 \mathrm{E}-02$ & $4.06 \mathrm{E}-02$ & $5.01 \mathrm{E}-02$ & $1.13 \mathrm{E}-01$ \\
\hline & 42 & $5.81 \mathrm{E}-02$ & $1.15 \mathrm{E}-02$ & $5.53 \mathrm{E}-02$ & $8.26 \mathrm{E}-02$ & 1.09E-01 & $1.35 \mathrm{E}-01$ & 3.09E-01 \\
\hline & 43 & $2.38 \mathrm{E}-04$ & $1.31 \mathrm{E}-03$ & $2.49 \mathrm{E}-04$ & $1.95 \mathrm{E}-04$ & $2.06 \mathrm{E}-04$ & 2.37E-04 & 5.11E-04 \\
\hline & 44 & $1.99 \mathrm{E}-02$ & 4.93E-03 & $1.89 \mathrm{E}-02$ & $2.82 \mathrm{E}-02$ & $3.74 \mathrm{E}-02$ & 4.64E-02 & 1.06E-01 \\
\hline & 45 & 4.35E-05 & $4.05 \mathrm{E}-04$ & 4.84E-05 & $1.74 \mathrm{E}-05$ & $7.04 \mathrm{E}-06$ & $3.02 \mathrm{E}-06$ & $0.00 E+00$ \\
\hline & 46 & $1.47 \mathrm{E}-05$ & $8.01 \mathrm{E}-06$ & $1.44 \mathrm{E}-05$ & $1.66 \mathrm{E}-05$ & $1.81 \mathrm{E}-05$ & $1.91 \mathrm{E}-05$ & $1.98 \mathrm{E}-05$ \\
\hline & 47 & 2.44E-05 & $1.98 \mathrm{E}-05$ & $2.41 \mathrm{E}-05$ & $2.70 \mathrm{E}-05$ & 2.92E-05 & 3.07E-05 & $3.18 \mathrm{E}-05$ \\
\hline & 48 & 5.67E-03 & $3.21 \mathrm{E}-03$ & $5.55 \mathrm{E}-03$ & $6.40 \mathrm{E}-03$ & $7.00 \mathrm{E}-03$ & $7.38 \mathrm{E}-03$ & 7.67E-03 \\
\hline & 49 & $1.66 \mathrm{E}-02$ & $9.36 \mathrm{E}-03$ & $1.62 \mathrm{E}-02$ & $1.87 \mathrm{E}-02$ & $2.04 \mathrm{E}-02$ & $2.16 \mathrm{E}-02$ & $2.24 \mathrm{E}-02$ \\
\hline & 50 & 4.91E-02 & $2.77 \mathrm{E}-02$ & $4.80 \mathrm{E}-02$ & $5.54 \mathrm{E}-02$ & $6.06 \mathrm{E}-02$ & $6.40 \mathrm{E}-02$ & $6.66 \mathrm{E}-02$ \\
\hline & 51 & 1.69E-02 & 1.10E-02 & $1.66 \mathrm{E}-02$ & $1.90 \mathrm{E}-02$ & 2.07E-02 & 2.19E-02 & 2.27E-02 \\
\hline & 52 & 2.49E-02 & $1.42 \mathrm{E}-02$ & $2.44 \mathrm{E}-02$ & $2.81 \mathrm{E}-02$ & $3.08 \mathrm{E}-02$ & $3.25 \mathrm{E}-02$ & 3.38E-02 \\
\hline & 53 & $2.27 \mathrm{E}-03$ & $2.90 \mathrm{E}-03$ & $2.26 \mathrm{E}-03$ & $2.41 \mathrm{E}-03$ & $2.58 \mathrm{E}-03$ & $2.70 \mathrm{E}-03$ & $2.79 \mathrm{E}-03$ \\
\hline & 54 & $2.05 \mathrm{E}-02$ & $1.26 \mathrm{E}-02$ & $2.01 \mathrm{E}-02$ & $2.30 \mathrm{E}-02$ & $2.52 \mathrm{E}-02$ & $2.66 \mathrm{E}-02$ & 2.77E-02 \\
\hline & 55 & 2.67E-04 & 1.17E-03 & $2.84 \mathrm{E}-04$ & 2.07E-04 & $1.86 \mathrm{E}-04$ & $1.83 \mathrm{E}-04$ & $1.83 \mathrm{E}-04$ \\
\hline & 56 & $1.63 \mathrm{E}-05$ & $1.31 \mathrm{E}-05$ & $1.61 \mathrm{E}-05$ & 1.77E-05 & $1.91 \mathrm{E}-05$ & $2.03 E-05$ & 2.67E-05 \\
\hline & 57 & 6.69E-05 & $4.66 \mathrm{E}-05$ & $6.53 \mathrm{E}-05$ & $7.55 \mathrm{E}-05$ & $8.49 \mathrm{E}-05$ & $9.40 \mathrm{E}-05$ & 1.50E-04 \\
\hline & 58 & 2.63E-04 & $2.18 \mathrm{E}-04$ & $2.62 \mathrm{E}-04$ & $2.74 \mathrm{E}-04$ & $2.83 \mathrm{E}-04$ & $2.89 \mathrm{E}-04$ & $2.96 \mathrm{E}-04$ \\
\hline & 59 & $1.14 \mathrm{E}-02$ & $9.21 \mathrm{E}-03$ & $1.13 \mathrm{E}-02$ & 1.19E-02 & $1.22 \mathrm{E}-02$ & $1.24 \mathrm{E}-02$ & 1.26E-02 \\
\hline & 60 & $9.01 \mathrm{E}-03$ & $1.20 \mathrm{E}-02$ & $9.06 \mathrm{E}-03$ & 8.99E-03 & $9.07 \mathrm{E}-03$ & $9.17 \mathrm{E}-03$ & $9.26 \mathrm{E}-03$ \\
\hline & 61 & $1.50 \mathrm{E}-02$ & $1.28 \mathrm{E}-02$ & $1.50 \mathrm{E}-02$ & $1.56 \mathrm{E}-02$ & $1.60 \mathrm{E}-02$ & $1.63 \mathrm{E}-02$ & $1.65 \mathrm{E}-02$ \\
\hline & 62 & 4.17E-02 & $3.38 \mathrm{E}-02$ & 4.16E-02 & $4.35 \mathrm{E}-02$ & $4.48 \mathrm{E}-02$ & $4.56 \mathrm{E}-02$ & 4.63E-02 \\
\hline & 63 & $2.56 \mathrm{E}-02$ & $2.08 \mathrm{E}-02$ & $2.55 \mathrm{E}-02$ & 2.67E-02 & $2.75 \mathrm{E}-02$ & $2.80 \mathrm{E}-02$ & $2.84 \mathrm{E}-02$ \\
\hline & 64 & $4.08 \mathrm{E}-03$ & $6.04 \mathrm{E}-03$ & $4.12 \mathrm{E}-03$ & $4.02 \mathrm{E}-03$ & $4.04 \mathrm{E}-03$ & 4.07E-03 & 4.11E-03 \\
\hline & 65 & $1.25 \mathrm{E}-03$ & $5.63 \mathrm{E}-03$ & $1.33 \mathrm{E}-03$ & $9.10 \mathrm{E}-04$ & $7.58 \mathrm{E}-04$ & 7.12E-04 & 7.07E-04 \\
\hline & 66 & $2.54 \mathrm{E}-02$ & $2.21 \mathrm{E}-02$ & $2.54 \mathrm{E}-02$ & $2.64 \mathrm{E}-02$ & $2.71 \mathrm{E}-02$ & $2.76 \mathrm{E}-02$ & 2.79E-02 \\
\hline & 67 & $1.28 \mathrm{E}-06$ & $1.06 \mathrm{E}-05$ & $1.46 \mathrm{E}-06$ & $5.74 \mathrm{E}-07$ & $2.44 \mathrm{E}-07$ & $9.46 \mathrm{E}-08$ & $0.00 E+00$ \\
\hline & 68 & $7.73 \mathrm{E}-07$ & $1.40 \mathrm{E}-06$ & $7.63 \mathrm{E}-07$ & $8.12 \mathrm{E}-07$ & $9.13 \mathrm{E}-07$ & $1.06 \mathrm{E}-06$ & $1.00 \mathrm{E}-06$ \\
\hline & 69 & $1.97 \mathrm{E}-05$ & $1.27 \mathrm{E}-05$ & $1.94 \mathrm{E}-05$ & $2.23 \mathrm{E}-05$ & $2.43 \mathrm{E}-05$ & $2.58 \mathrm{E}-05$ & 2.81E-05 \\
\hline & 70 & $6.91 \mathrm{E}-06$ & $4.56 \mathrm{E}-06$ & $6.76 \mathrm{E}-06$ & $7.96 \mathrm{E}-06$ & $8.81 \mathrm{E}-06$ & $9.39 \mathrm{E}-06$ & $9.99 \mathrm{E}-06$ \\
\hline & 71 & 6.19E-06 & $1.58 \mathrm{E}-05$ & $6.35 \mathrm{E}-06$ & 5.91E-06 & $6.00 \mathrm{E}-06$ & $6.23 \mathrm{E}-06$ & $6.53 \mathrm{E}-06$ \\
\hline & 72 & $1.40 \mathrm{E}-06$ & 1.69E-06 & $1.40 \mathrm{E}-06$ & $1.50 \mathrm{E}-06$ & $1.60 \mathrm{E}-06$ & $1.68 \mathrm{E}-06$ & $2.24 \mathrm{E}-06$ \\
\hline & 73 & $3.79 \mathrm{E}-06$ & 3.56E-06 & $3.81 \mathrm{E}-06$ & 3.96E-06 & 4.06E-06 & 4.04E-06 & 4.12E-06 \\
\hline & 74 & $4.13 \mathrm{E}-06$ & 3.07E-06 & $4.08 \mathrm{E}-06$ & 4.71E-06 & 5.31E-06 & $5.80 \mathrm{E}-06$ & $9.30 \mathrm{E}-06$ \\
\hline & 75 & 2.47E-05 & $1.15 \mathrm{E}-05$ & 2.39E-05 & $3.12 \mathrm{E}-05$ & $3.85 \mathrm{E}-05$ & 4.54E-05 & $9.20 \mathrm{E}-05$ \\
\hline & 76 & $2.78 \mathrm{E}-02$ & $2.20 \mathrm{E}-02$ & $2.76 \mathrm{E}-02$ & $2.82 \mathrm{E}-02$ & $2.86 \mathrm{E}-02$ & $2.93 \mathrm{E}-02$ & 2.79E-02 \\
\hline & 77 & $9.64 \mathrm{E}-05$ & $5.73 \mathrm{E}-04$ & 1.07E-04 & 4.20E-05 & $1.72 \mathrm{E}-05$ & $8.80 \mathrm{E}-06$ & 6.19E-07 \\
\hline & 78 & 4.10E-04 & $1.55 \mathrm{E}-04$ & 4.03E-04 & $5.86 \mathrm{E}-04$ & $7.58 \mathrm{E}-04$ & $9.23 \mathrm{E}-04$ & $2.04 \mathrm{E}-03$ \\
\hline & 79 & $4.83 \mathrm{E}-07$ & $2.25 \mathrm{E}-06$ & 5.33E-07 & 2.71E-07 & $1.42 \mathrm{E}-07$ & 8.04E-08 & $0.00 E+00$ \\
\hline & 80 & $4.54 \mathrm{E}-03$ & $2.15 \mathrm{E}-03$ & $4.49 \mathrm{E}-03$ & $6.48 \mathrm{E}-03$ & $8.38 \mathrm{E}-03$ & $1.02 \mathrm{E}-02$ & $2.26 \mathrm{E}-02$ \\
\hline & 81 & $4.95 \mathrm{E}-03$ & $1.89 \mathrm{E}-03$ & $4.88 \mathrm{E}-03$ & 7.11E-03 & $9.23 \mathrm{E}-03$ & $1.13 \mathrm{E}-02$ & $2.50 \mathrm{E}-02$ \\
\hline & 82 & $1.24 \mathrm{E}-02$ & $4.66 \mathrm{E}-03$ & $1.22 \mathrm{E}-02$ & $1.79 \mathrm{E}-02$ & $2.33 \mathrm{E}-02$ & $2.85 \mathrm{E}-02$ & 6.39E-02 \\
\hline & 83 & $3.66 \mathrm{E}-04$ & $6.06 \mathrm{E}-04$ & $3.68 \mathrm{E}-04$ & $4.72 \mathrm{E}-04$ & $6.03 \mathrm{E}-04$ & $7.25 \mathrm{E}-04$ & $1.63 \mathrm{E}-03$ \\
\hline & 84 & 4.43E-03 & $2.10 \mathrm{E}-03$ & $4.35 \mathrm{E}-03$ & $6.32 \mathrm{E}-03$ & $8.23 \mathrm{E}-03$ & $1.01 \mathrm{E}-02$ & $2.26 \mathrm{E}-02$ \\
\hline & 85 & $1.96 \mathrm{E}-05$ & $1.85 \mathrm{E}-04$ & $2.28 \mathrm{E}-05$ & 7.99E-06 & $3.05 \mathrm{E}-06$ & $1.34 \mathrm{E}-06$ & $0.00 E+00$ \\
\hline & 86 & $3.02 \mathrm{E}-04$ & $1.88 \mathrm{E}-04$ & $2.96 \mathrm{E}-04$ & 3.37E-04 & $3.67 \mathrm{E}-04$ & $3.89 \mathrm{E}-04$ & 4.00E-04 \\
\hline & 87 & 8.14E-04 & 5.11E-04 & 8.00E-04 & $9.08 \mathrm{E}-04$ & $9.90 \mathrm{E}-04$ & $1.05 \mathrm{E}-03$ & $1.08 \mathrm{E}-03$ \\
\hline & 88 & $1.06 \mathrm{E}-03$ & $6.70 \mathrm{E}-04$ & $1.04 \mathrm{E}-03$ & $1.18 \mathrm{E}-03$ & $1.29 \mathrm{E}-03$ & $1.36 \mathrm{E}-03$ & 1.40E-03 \\
\hline & 89 & 3.10E-03 & $1.96 \mathrm{E}-03$ & $3.04 \mathrm{E}-03$ & $3.45 \mathrm{E}-03$ & $3.76 \mathrm{E}-03$ & $3.98 \mathrm{E}-03$ & 4.08E-03 \\
\hline & 90 & $5.16 \mathrm{E}-03$ & $3.32 \mathrm{E}-03$ & $5.06 \mathrm{E}-03$ & $5.74 \mathrm{E}-03$ & $6.25 \mathrm{E}-03$ & $6.62 \mathrm{E}-03$ & 6.81E-03 \\
\hline & 91 & 4.16E-03 & 3.19E-03 & $4.10 \mathrm{E}-03$ & $4.58 \mathrm{E}-03$ & $4.96 \mathrm{E}-03$ & $5.24 \mathrm{E}-03$ & $5.38 \mathrm{E}-03$ \\
\hline & 92 & $1.20 \mathrm{E}-02$ & $7.54 \mathrm{E}-03$ & $1.18 \mathrm{E}-02$ & $1.34 \mathrm{E}-02$ & $1.45 \mathrm{E}-02$ & $1.54 \mathrm{E}-02$ & 1.59E-02 \\
\hline & 93 & $1.05 \mathrm{E}-03$ & $1.26 \mathrm{E}-03$ & $1.04 \mathrm{E}-03$ & $1.11 \mathrm{E}-03$ & $1.18 \mathrm{E}-03$ & $1.24 \mathrm{E}-03$ & $1.26 \mathrm{E}-03$ \\
\hline & 94 & 5.19E-03 & 3.70E-03 & 5.11E-03 & $5.74 \mathrm{E}-03$ & $6.24 \mathrm{E}-03$ & $6.60 \mathrm{E}-03$ & $6.80 \mathrm{E}-03$ \\
\hline & 95 & $5.94 \mathrm{E}-04$ & $4.23 \mathrm{E}-04$ & $5.86 \mathrm{E}-04$ & $6.56 \mathrm{E}-04$ & 7.09E-04 & 7.49E-04 & 7.51E-04 \\
\hline & 96 & $1.50 \mathrm{E}-04$ & $2.08 \mathrm{E}-04$ & $1.50 \mathrm{E}-04$ & $1.56 \mathrm{E}-04$ & 1.64E-04 & $1.72 \mathrm{E}-04$ & $1.75 \mathrm{E}-04$ \\
\hline & 97 & 4.95E-05 & $3.53 \mathrm{E}-04$ & 5.51E-05 & 2.19E-05 & $8.75 \mathrm{E}-06$ & $3.86 \mathrm{E}-06$ & $1.42 \mathrm{E}-06$ \\
\hline & 98 & $1.57 \mathrm{E}-03$ & $1.44 \mathrm{E}-03$ & $1.56 \mathrm{E}-03$ & 1.67E-03 & 1.79E-03 & $1.87 \mathrm{E}-03$ & $1.92 \mathrm{E}-03$ \\
\hline & 99 & $2.26 \mathrm{E}-03$ & $2.24 \mathrm{E}-03$ & $2.24 \mathrm{E}-03$ & 2.39E-03 & $2.54 \mathrm{E}-03$ & $2.65 \mathrm{E}-03$ & $2.73 \mathrm{E}-03$ \\
\hline & 100 & $1.48 \mathrm{E}-03$ & $2.78 \mathrm{E}-03$ & $1.50 \mathrm{E}-03$ & $1.43 \mathrm{E}-03$ & $1.46 \mathrm{E}-03$ & $1.50 \mathrm{E}-03$ & $1.52 \mathrm{E}-03$ \\
\hline
\end{tabular}


Table 4 (continued)

\begin{tabular}{|c|c|c|c|c|c|c|c|c|}
\hline \multirow[b]{2}{*}{$\mathrm{i}$} & \multirow[b]{2}{*}{ j } & \multicolumn{7}{|c|}{ collision strengths at energies } \\
\hline & & $2944.1 \mathrm{eV}$ & $4868.3 \mathrm{eV}$ & $7564.6 \mathrm{eV}$ & $11040 \mathrm{eV}$ & $15221 \mathrm{eV}$ & $65.33 \mathrm{eV}$ & $1714.98 \mathrm{eV}$ \\
\hline & 101 & $5.83 \mathrm{E}-03$ & $5.35 \mathrm{E}-03$ & $5.79 \mathrm{E}-03$ & $6.21 \mathrm{E}-03$ & $6.63 \mathrm{E}-03$ & $6.93 \mathrm{E}-03$ & $7.10 \mathrm{E}-03$ \\
\hline & 102 & $3.16 \mathrm{E}-03$ & $3.13 \mathrm{E}-03$ & $3.14 \mathrm{E}-03$ & $3.34 \mathrm{E}-03$ & $3.56 \mathrm{E}-03$ & $3.73 \mathrm{E}-03$ & $3.83 \mathrm{E}-03$ \\
\hline & 103 & $1.84 \mathrm{E}-03$ & $1.49 \mathrm{E}-03$ & $1.74 \mathrm{E}-03$ & $1.95 \mathrm{E}-03$ & 2.09E-03 & $2.28 \mathrm{E}-03$ & $2.18 \mathrm{E}-03$ \\
\hline & 104 & $1.22 \mathrm{E}-03$ & $1.53 \mathrm{E}-03$ & 1.16E-03 & $1.25 \mathrm{E}-03$ & $1.32 \mathrm{E}-03$ & $1.44 \mathrm{E}-03$ & 1.37E-03 \\
\hline & 105 & 7.59E-04 & $1.95 \mathrm{E}-03$ & $7.82 \mathrm{E}-04$ & $6.84 \mathrm{E}-04$ & $6.71 \mathrm{E}-04$ & $6.76 \mathrm{E}-04$ & $6.82 \mathrm{E}-04$ \\
\hline & 106 & 5.36E-04 & $1.40 \mathrm{E}-03$ & $5.53 \mathrm{E}-04$ & $4.81 \mathrm{E}-04$ & 4.71E-04 & $4.81 \mathrm{E}-04$ & 4.76E-04 \\
\hline & 107 & 3.57E-03 & $3.78 \mathrm{E}-03$ & $3.55 \mathrm{E}-03$ & $3.75 \mathrm{E}-03$ & $3.98 \mathrm{E}-03$ & 4.16E-03 & $4.25 \mathrm{E}-03$ \\
\hline & 108 & $1.02 \mathrm{E}-03$ & $2.02 \mathrm{E}-03$ & $9.85 \mathrm{E}-04$ & $9.90 \mathrm{E}-04$ & $1.02 \mathrm{E}-03$ & $1.11 \mathrm{E}-03$ & $1.05 \mathrm{E}-03$ \\
\hline & 109 & $1.43 \mathrm{E}-03$ & $1.60 \mathrm{E}-03$ & $1.36 \mathrm{E}-03$ & $1.49 \mathrm{E}-03$ & $1.58 \mathrm{E}-03$ & $1.72 \mathrm{E}-03$ & $1.64 \mathrm{E}-03$ \\
\hline & 110 & 4.72E-03 & $3.82 \mathrm{E}-03$ & $4.46 \mathrm{E}-03$ & 5.01E-03 & 5.35E-03 & $5.86 \mathrm{E}-03$ & 5.59E-03 \\
\hline & 111 & $2.48 \mathrm{E}-03$ & $2.31 \mathrm{E}-03$ & $2.35 \mathrm{E}-03$ & $2.61 \mathrm{E}-03$ & $2.78 \mathrm{E}-03$ & $3.04 \mathrm{E}-03$ & $2.90 \mathrm{E}-03$ \\
\hline & 112 & $8.85 \mathrm{E}-04$ & $1.65 \mathrm{E}-03$ & $8.56 \mathrm{E}-04$ & $8.71 \mathrm{E}-04$ & $9.03 \mathrm{E}-04$ & $9.81 \mathrm{E}-04$ & $9.28 \mathrm{E}-04$ \\
\hline & 113 & $3.08 \mathrm{E}-03$ & 2.83E-03 & $2.92 \mathrm{E}-03$ & $3.25 \mathrm{E}-03$ & $3.46 \mathrm{E}-03$ & 3.79E-03 & $3.61 \mathrm{E}-03$ \\
\hline & 114 & $6.47 \mathrm{E}-08$ & $4.33 \mathrm{E}-08$ & $6.35 \mathrm{E}-08$ & $7.78 \mathrm{E}-08$ & 8.84E-08 & 8.99E-08 & $1.07 \mathrm{E}-07$ \\
\hline & 115 & 2.93E-07 & $1.01 \mathrm{E}-06$ & $3.05 E-07$ & $2.48 \mathrm{E}-07$ & $2.38 \mathrm{E}-07$ & $2.34 \mathrm{E}-07$ & $2.57 \mathrm{E}-07$ \\
\hline & 116 & $6.06 \mathrm{E}-06$ & 5.07E-06 & $6.11 \mathrm{E}-06$ & $6.51 \mathrm{E}-06$ & 8.09E-06 & 7.37E-06 & $7.25 \mathrm{E}-06$ \\
\hline & 117 & $9.91 \mathrm{E}-06$ & 8.93E-06 & $9.92 \mathrm{E}-06$ & $1.07 \mathrm{E}-05$ & $2.26 \mathrm{E}-05$ & $1.21 \mathrm{E}-05$ & $1.40 \mathrm{E}-05$ \\
\hline & 118 & $1.36 \mathrm{E}-06$ & $1.34 \mathrm{E}-06$ & $1.36 \mathrm{E}-06$ & $1.43 \mathrm{E}-06$ & $7.94 \mathrm{E}-06$ & $1.54 \mathrm{E}-06$ & $1.57 \mathrm{E}-06$ \\
\hline & 119 & 1.67E-07 & 1.43E-06 & $1.87 \mathrm{E}-07$ & 7.49E-08 & $3.27 \mathrm{E}-08$ & $1.33 \mathrm{E}-08$ & $0.00 \mathrm{E}+00$ \\
\hline & 120 & $1.91 \mathrm{E}-05$ & $1.81 \mathrm{E}-05$ & $1.88 \mathrm{E}-05$ & $2.12 \mathrm{E}-05$ & 2.44E-05 & 2.61E-05 & $4.46 \mathrm{E}-05$ \\
\hline & 121 & $1.42 \mathrm{E}-05$ & $1.24 \mathrm{E}-05$ & $1.43 \mathrm{E}-05$ & $1.53 \mathrm{E}-05$ & $1.70 \mathrm{E}-05$ & $1.15 \mathrm{E}-05$ & $1.66 \mathrm{E}-05$ \\
\hline & 122 & $1.51 \mathrm{E}-05$ & $1.40 \mathrm{E}-05$ & $1.52 \mathrm{E}-05$ & $1.63 \mathrm{E}-05$ & $2.08 \mathrm{E}-05$ & $1.65 \mathrm{E}-05$ & $1.94 \mathrm{E}-05$ \\
\hline & 123 & $9.28 \mathrm{E}-07$ & 4.15E-06 & $9.94 \mathrm{E}-07$ & $7.00 \mathrm{E}-07$ & $1.57 \mathrm{E}-06$ & 4.83E-07 & $5.80 \mathrm{E}-07$ \\
\hline & 124 & 2.09E-07 & $2.10 \mathrm{E}-07$ & $1.15 \mathrm{E}-07$ & $4.68 \mathrm{E}-07$ & $1.60 \mathrm{E}-08$ & $4.48 \mathrm{E}-07$ & $1.96 \mathrm{E}-07$ \\
\hline & 125 & $4.18 \mathrm{E}-06$ & $2.48 \mathrm{E}-06$ & $3.24 \mathrm{E}-06$ & $3.60 \mathrm{E}-06$ & $3.88 \mathrm{E}-06$ & 5.93E-06 & 2.99E-06 \\
\hline & 126 & 4.47E-07 & $6.70 \mathrm{E}-07$ & $4.50 \mathrm{E}-07$ & 5.39E-07 & $3.23 \mathrm{E}-07$ & $5.58 \mathrm{E}-07$ & $5.64 \mathrm{E}-07$ \\
\hline & 127 & $1.37 \mathrm{E}-05$ & $1.29 \mathrm{E}-05$ & $1.35 \mathrm{E}-05$ & $1.51 \mathrm{E}-05$ & $1.66 \mathrm{E}-05$ & $1.74 \mathrm{E}-05$ & $1.86 \mathrm{E}-05$ \\
\hline & 128 & $8.00 \mathrm{E}-05$ & $4.72 \mathrm{E}-05$ & $7.80 \mathrm{E}-05$ & $9.03 E-05$ & $1.01 \mathrm{E}-04$ & $1.07 \mathrm{E}-04$ & $1.14 \mathrm{E}-04$ \\
\hline & 129 & 4.47E-07 & 1.89E-06 & $4.83 E-07$ & 3.19E-07 & $2.70 \mathrm{E}-07$ & $2.55 \mathrm{E}-07$ & $2.57 \mathrm{E}-07$ \\
\hline & 130 & 4.74E-06 & $5.02 E-06$ & $4.72 \mathrm{E}-06$ & $5.23 \mathrm{E}-06$ & $5.62 \mathrm{E}-06$ & $5.56 \mathrm{E}-06$ & $6.25 \mathrm{E}-06$ \\
\hline & 131 & $9.29 E-06$ & 8.87E-06 & $9.14 \mathrm{E}-06$ & $1.02 \mathrm{E}-05$ & $1.13 \mathrm{E}-05$ & $1.16 \mathrm{E}-05$ & $1.26 \mathrm{E}-05$ \\
\hline & 132 & $1.13 \mathrm{E}-07$ & $2.21 \mathrm{E}-07$ & $1.13 \mathrm{E}-07$ & $1.26 \mathrm{E}-07$ & $1.87 \mathrm{E}-07$ & $-6.05 E-07$ & $2.54 \mathrm{E}-07$ \\
\hline & 133 & $1.68 \mathrm{E}-07$ & $3.54 \mathrm{E}-07$ & $1.72 \mathrm{E}-07$ & $1.68 \mathrm{E}-07$ & $2.48 \mathrm{E}-07$ & 2.09E-07 & $1.72 \mathrm{E}-07$ \\
\hline & 134 & 4.14E-07 & 3.87E-07 & $3.98 \mathrm{E}-07$ & $5.27 \mathrm{E}-07$ & $7.30 \mathrm{E}-07$ & 5.89E-07 & $1.86 \mathrm{E}-06$ \\
\hline & 135 & $4.45 \mathrm{E}-06$ & $1.84 \mathrm{E}-06$ & 4.19E-06 & $6.00 \mathrm{E}-06$ & 7.99E-06 & $9.81 \mathrm{E}-06$ & $2.32 \mathrm{E}-05$ \\
\hline & 136 & $4.43 \mathrm{E}-08$ & 9.39E-08 & 4.10E-08 & 4.64E-08 & $5.18 \mathrm{E}-08$ & $5.23 \mathrm{E}-08$ & $5.61 \mathrm{E}-08$ \\
\hline & 137 & $2.79 \mathrm{E}-08$ & $1.24 \mathrm{E}-07$ & $2.72 \mathrm{E}-08$ & $2.49 \mathrm{E}-08$ & $2.31 \mathrm{E}-08$ & $2.34 \mathrm{E}-08$ & $2.63 \mathrm{E}-08$ \\
\hline & 138 & $5.41 \mathrm{E}-07$ & 7.79E-07 & 5.31E-07 & $5.60 \mathrm{E}-07$ & $6.33 \mathrm{E}-07$ & 5.49E-07 & $6.57 \mathrm{E}-07$ \\
\hline & 139 & $4.53 \mathrm{E}-06$ & $3.02 \mathrm{E}-06$ & $4.36 \mathrm{E}-06$ & 4.99E-06 & $5.63 \mathrm{E}-06$ & 5.79E-06 & $6.21 \mathrm{E}-06$ \\
\hline & 140 & $1.03 \mathrm{E}-02$ & $7.95 \mathrm{E}-03$ & $1.01 \mathrm{E}-02$ & $1.02 \mathrm{E}-02$ & $1.04 \mathrm{E}-02$ & $1.04 \mathrm{E}-02$ & $9.93 \mathrm{E}-03$ \\
\hline & 141 & 4.97E-05 & 2.97E-04 & 5.49E-05 & $2.15 E-05$ & $8.51 \mathrm{E}-06$ & $4.18 \mathrm{E}-06$ & $1.80 \mathrm{E}-07$ \\
\hline & 142 & $2.11 \mathrm{E}-03$ & $8.31 \mathrm{E}-04$ & $2.16 \mathrm{E}-03$ & $3.20 \mathrm{E}-03$ & $4.12 \mathrm{E}-03$ & $5.02 \mathrm{E}-03$ & $1.05 \mathrm{E}-02$ \\
\hline & 143 & $1.77 \mathrm{E}-03$ & $9.19 E-04$ & $1.82 \mathrm{E}-03$ & $2.66 \mathrm{E}-03$ & $3.42 \mathrm{E}-03$ & $4.15 \mathrm{E}-03$ & $8.62 \mathrm{E}-03$ \\
\hline & 144 & $4.71 \mathrm{E}-03$ & $1.88 \mathrm{E}-03$ & $4.82 \mathrm{E}-03$ & 7.19E-03 & $9.29 \mathrm{E}-03$ & $1.13 \mathrm{E}-02$ & $2.38 \mathrm{E}-02$ \\
\hline & 145 & $5.94 \mathrm{E}-05$ & 2.77E-04 & $6.55 \mathrm{E}-05$ & 5.71E-05 & $6.30 \mathrm{E}-05$ & 7.37E-05 & $1.62 \mathrm{E}-04$ \\
\hline & 146 & $1.70 \mathrm{E}-03$ & $9.02 \mathrm{E}-04$ & $1.75 \mathrm{E}-03$ & $2.58 \mathrm{E}-03$ & $3.32 \mathrm{E}-03$ & 4.04E-03 & 8.39E-03 \\
\hline & 147 & $1.13 \mathrm{E}-05$ & $1.03 \mathrm{E}-04$ & $1.33 \mathrm{E}-05$ & $4.68 \mathrm{E}-06$ & $1.73 \mathrm{E}-06$ & $7.55 \mathrm{E}-07$ & $0.00 E+00$ \\
\hline & 148 & $5.36 \mathrm{E}-04$ & $3.51 \mathrm{E}-04$ & $5.25 \mathrm{E}-04$ & $5.98 \mathrm{E}-04$ & $6.45 \mathrm{E}-04$ & $6.88 \mathrm{E}-04$ & $6.96 \mathrm{E}-04$ \\
\hline & 149 & $1.60 \mathrm{E}-03$ & $1.05 \mathrm{E}-03$ & $1.57 \mathrm{E}-03$ & $1.78 \mathrm{E}-03$ & $1.92 \mathrm{E}-03$ & $2.05 \mathrm{E}-03$ & $2.08 \mathrm{E}-03$ \\
\hline & 150 & $1.69 \mathrm{E}-03$ & $1.41 \mathrm{E}-03$ & $1.67 \mathrm{E}-03$ & $1.86 \mathrm{E}-03$ & 1.99E-03 & $2.12 \mathrm{E}-03$ & $2.14 \mathrm{E}-03$ \\
\hline & 151 & $4.78 \mathrm{E}-03$ & $3.12 \mathrm{E}-03$ & $4.68 \mathrm{E}-03$ & $5.33 \mathrm{E}-03$ & $5.76 \mathrm{E}-03$ & $6.13 \mathrm{E}-03$ & $6.22 \mathrm{E}-03$ \\
\hline & 152 & $2.63 \mathrm{E}-03$ & $1.73 \mathrm{E}-03$ & $2.58 \mathrm{E}-03$ & $2.94 \mathrm{E}-03$ & $3.17 \mathrm{E}-03$ & $3.38 \mathrm{E}-03$ & $3.42 \mathrm{E}-03$ \\
\hline & 153 & $9.79 E-05$ & $4.40 \mathrm{E}-04$ & $1.03 E-04$ & 7.04E-05 & $5.93 \mathrm{E}-05$ & $5.65 \mathrm{E}-05$ & 5.37E-05 \\
\hline & 154 & $2.12 \mathrm{E}-03$ & $1.62 \mathrm{E}-03$ & $2.08 \mathrm{E}-03$ & $2.34 \mathrm{E}-03$ & $2.52 \mathrm{E}-03$ & $2.68 \mathrm{E}-03$ & $2.70 \mathrm{E}-03$ \\
\hline & 155 & $4.48 \mathrm{E}-05$ & $2.75 \mathrm{E}-04$ & $4.85 \mathrm{E}-05$ & 2.47E-05 & $1.57 \mathrm{E}-05$ & $1.24 \mathrm{E}-05$ & $1.03 \mathrm{E}-05$ \\
\hline & 156 & $5.18 \mathrm{E}-04$ & $5.18 \mathrm{E}-04$ & $5.12 \mathrm{E}-04$ & 5.09E-04 & $5.95 \mathrm{E}-04$ & 6.07E-04 & $6.56 \mathrm{E}-04$ \\
\hline & 157 & $5.31 \mathrm{E}-04$ & $1.18 \mathrm{E}-03$ & $5.40 \mathrm{E}-04$ & 4.62E-04 & $5.01 \mathrm{E}-04$ & $5.02 \mathrm{E}-04$ & $5.35 \mathrm{E}-04$ \\
\hline & 158 & $7.75 \mathrm{E}-04$ & $8.34 \mathrm{E}-04$ & 7.67E-04 & 7.51E-04 & $8.81 \mathrm{E}-04$ & 8.96E-04 & $9.71 \mathrm{E}-04$ \\
\hline & 159 & $1.90 \mathrm{E}-03$ & $1.90 \mathrm{E}-03$ & $1.88 \mathrm{E}-03$ & $1.84 \mathrm{E}-03$ & 2.17E-03 & $2.22 \mathrm{E}-03$ & $2.41 \mathrm{E}-03$ \\
\hline & 160 & $1.20 \mathrm{E}-03$ & $1.21 \mathrm{E}-03$ & 1.19E-03 & $1.16 \mathrm{E}-03$ & $1.37 \mathrm{E}-03$ & $1.40 \mathrm{E}-03$ & $1.52 \mathrm{E}-03$ \\
\hline & 161 & $1.32 \mathrm{E}-04$ & 8.13E-04 & 1.47E-04 & $7.03 E-05$ & 4.57E-05 & $3.13 \mathrm{E}-05$ & 2.79E-05 \\
\hline & 162 & $1.74 \mathrm{E}-04$ & $6.18 \mathrm{E}-04$ & $1.83 \mathrm{E}-04$ & $1.31 \mathrm{E}-04$ & $1.26 \mathrm{E}-04$ & $1.23 \mathrm{E}-04$ & $1.27 \mathrm{E}-04$ \\
\hline & 163 & $1.18 \mathrm{E}-03$ & $1.42 \mathrm{E}-03$ & 1.17E-03 & $1.11 \mathrm{E}-03$ & $1.31 \mathrm{E}-03$ & $1.33 \mathrm{E}-03$ & $1.44 \mathrm{E}-03$ \\
\hline & 164 & $9.21 \mathrm{E}-04$ & $7.72 \mathrm{E}-04$ & $8.52 \mathrm{E}-04$ & $9.73 \mathrm{E}-04$ & $1.05 \mathrm{E}-03$ & $-2.04 \mathrm{E}-04$ & $1.12 \mathrm{E}-03$ \\
\hline & 165 & $5.45 \mathrm{E}-04$ & $1.24 \mathrm{E}-03$ & $5.22 \mathrm{E}-04$ & $5.15 \mathrm{E}-04$ & $5.28 \mathrm{E}-04$ & $-5.10 \mathrm{E}-05$ & $5.50 \mathrm{E}-04$ \\
\hline & 166 & $1.68 \mathrm{E}-04$ & $1.66 \mathrm{E}-04$ & $1.69 \mathrm{E}-04$ & $1.78 \mathrm{E}-04$ & $1.85 \mathrm{E}-04$ & $1.92 \mathrm{E}-04$ & $1.81 \mathrm{E}-04$ \\
\hline & 167 & $9.82 \mathrm{E}-05$ & $1.83 \mathrm{E}-04$ & $1.00 \mathrm{E}-04$ & $1.00 \mathrm{E}-04$ & $1.03 \mathrm{E}-04$ & $1.07 E-04$ & $1.00 \mathrm{E}-04$ \\
\hline & 168 & $8.06 \mathrm{E}-05$ & $2.46 \mathrm{E}-04$ & $8.34 \mathrm{E}-05$ & 7.81E-05 & $7.86 \mathrm{E}-05$ & $8.08 \mathrm{E}-05$ & $7.58 \mathrm{E}-05$ \\
\hline
\end{tabular}


Table 4 (continued)

\begin{tabular}{|c|c|c|c|c|c|c|c|c|}
\hline \multirow[b]{2}{*}{$\mathrm{i}$} & \multirow[b]{2}{*}{$\mathrm{j}$} & \multicolumn{7}{|c|}{ collision strengths at energies } \\
\hline & & $2944.1 \mathrm{eV}$ & $4868.3 \mathrm{eV}$ & $7564.6 \mathrm{eV}$ & $11040 \mathrm{eV}$ & $15221 \mathrm{eV}$ & $65.33 \mathrm{eV}$ & $1714.98 \mathrm{eV}$ \\
\hline & 169 & $2.39 \mathrm{E}-03$ & $2.00 \mathrm{E}-03$ & $2.21 \mathrm{E}-03$ & $2.52 \mathrm{E}-03$ & $2.72 \mathrm{E}-03$ & $-1.91 \mathrm{E}-03$ & $2.90 \mathrm{E}-03$ \\
\hline & 170 & $7.60 \mathrm{E}-04$ & $9.15 \mathrm{E}-04$ & 7.11E-04 & $7.81 \mathrm{E}-04$ & 8.33E-04 & $-3.56 \mathrm{E}-04$ & 8.84E-04 \\
\hline & 171 & 3.57E-04 & 3.54E-04 & $3.60 \mathrm{E}-04$ & $3.78 \mathrm{E}-04$ & $3.93 \mathrm{E}-04$ & $4.08 \mathrm{E}-04$ & $3.85 \mathrm{E}-04$ \\
\hline & 172 & $1.24 \mathrm{E}-04$ & $1.86 \mathrm{E}-04$ & $1.26 \mathrm{E}-04$ & $1.29 \mathrm{E}-04$ & $1.33 \mathrm{E}-04$ & $1.38 \mathrm{E}-04$ & $1.30 \mathrm{E}-04$ \\
\hline & 173 & $2.48 \mathrm{E}-04$ & $2.53 \mathrm{E}-04$ & $2.50 \mathrm{E}-04$ & $2.62 \mathrm{E}-04$ & $2.72 \mathrm{E}-04$ & $2.82 \mathrm{E}-04$ & $2.66 \mathrm{E}-04$ \\
\hline & 174 & $5.94 \mathrm{E}-04$ & $8.60 \mathrm{E}-04$ & $5.57 \mathrm{E}-04$ & $5.99 \mathrm{E}-04$ & $6.33 \mathrm{E}-04$ & $-5.93 E-05$ & $6.68 \mathrm{E}-04$ \\
\hline & 175 & $2.26 \mathrm{E}-05$ & $1.93 \mathrm{E}-04$ & $2.49 E-05$ & $1.62 \mathrm{E}-05$ & $1.42 \mathrm{E}-05$ & $1.40 \mathrm{E}-05$ & $1.30 \mathrm{E}-05$ \\
\hline & 176 & $1.37 \mathrm{E}-03$ & 1.29E-03 & $1.27 \mathrm{E}-03$ & $1.44 \mathrm{E}-03$ & $1.54 \mathrm{E}-03$ & $-4.43 E-04$ & $1.64 \mathrm{E}-03$ \\
\hline & 177 & $2.39 \mathrm{E}-04$ & $2.84 \mathrm{E}-04$ & $2.41 \mathrm{E}-04$ & $2.51 \mathrm{E}-04$ & $2.60 \mathrm{E}-04$ & $2.69 \mathrm{E}-04$ & $2.54 \mathrm{E}-04$ \\
\hline & 178 & $3.61 \mathrm{E}-04$ & $9.79 E-04$ & $3.50 \mathrm{E}-04$ & $3.29 E-04$ & $3.32 \mathrm{E}-04$ & $-3.13 E-04$ & $3.43 \mathrm{E}-04$ \\
\hline & 179 & $1.57 \mathrm{E}-03$ & $1.54 \mathrm{E}-03$ & $1.46 \mathrm{E}-03$ & $1.64 \mathrm{E}-03$ & $1.76 \mathrm{E}-03$ & $-1.20 \mathrm{E}-03$ & $1.88 \mathrm{E}-03$ \\
\hline & 180 & $1.34 \mathrm{E}-08$ & 8.84E-09 & 6.69E-09 & $9.98 \mathrm{E}-09$ & $1.83 \mathrm{E}-08$ & $1.86 \mathrm{E}-08$ & $9.33 \mathrm{E}-08$ \\
\hline & 181 & 3.87E-08 & $1.15 \mathrm{E}-07$ & $2.92 \mathrm{E}-08$ & $2.51 \mathrm{E}-08$ & $3.49 E-08$ & $3.44 \mathrm{E}-08$ & $1.55 \mathrm{E}-07$ \\
\hline & 182 & $1.22 \mathrm{E}-07$ & $1.10 \mathrm{E}-07$ & $1.12 \mathrm{E}-07$ & $1.46 \mathrm{E}-07$ & $1.55 \mathrm{E}-07$ & $1.77 \mathrm{E}-07$ & $2.01 \mathrm{E}-07$ \\
\hline & 183 & $1.00 \mathrm{E}-07$ & $1.63 \mathrm{E}-07$ & $1.01 \mathrm{E}-07$ & $1.30 \mathrm{E}-07$ & $1.46 \mathrm{E}-07$ & $1.53 \mathrm{E}-07$ & 4.90E-07 \\
\hline & 184 & $3.12 \mathrm{E}-07$ & 3.17E-07 & $2.89 \mathrm{E}-07$ & $3.11 \mathrm{E}-07$ & 3.47E-07 & 3.79E-07 & $9.19 \mathrm{E}-07$ \\
\hline & 185 & $7.05 \mathrm{E}-08$ & $6.29 \mathrm{E}-07$ & $8.02 \mathrm{E}-08$ & 3.09E-08 & $1.33 \mathrm{E}-08$ & 5.50E-09 & $0.00 \mathrm{E}+00$ \\
\hline & 186 & $7.50 \mathrm{E}-08$ & $9.25 \mathrm{E}-08$ & $3.54 \mathrm{E}-08$ & 4.07E-08 & $2.78 \mathrm{E}-08$ & $9.01 \mathrm{E}-08$ & $1.75 \mathrm{E}-07$ \\
\hline & 187 & $1.95 \mathrm{E}-06$ & 9.09E-07 & 1.17E-06 & 1.47E-06 & $1.56 \mathrm{E}-06$ & $1.66 \mathrm{E}-06$ & $3.05 \mathrm{E}-06$ \\
\hline & 188 & $1.33 \mathrm{E}-07$ & $1.89 \mathrm{E}-07$ & $1.38 \mathrm{E}-07$ & $1.48 \mathrm{E}-07$ & $1.33 \mathrm{E}-07$ & $1.64 \mathrm{E}-07$ & $1.76 \mathrm{E}-07$ \\
\hline & 189 & $2.03 \mathrm{E}-07$ & $9.02 \mathrm{E}-07$ & $2.24 \mathrm{E}-07$ & $1.46 \mathrm{E}-07$ & $1.21 \mathrm{E}-07$ & $1.07 \mathrm{E}-07$ & $1.13 \mathrm{E}-07$ \\
\hline & 190 & $4.96 \mathrm{E}-08$ & $1.08 \mathrm{E}-07$ & $5.26 \mathrm{E}-08$ & $2.29 \mathrm{E}-07$ & $3.34 \mathrm{E}-08$ & $6.62 \mathrm{E}-08$ & $1.55 \mathrm{E}-07$ \\
\hline & 191 & $1.12 \mathrm{E}-06$ & $6.00 \mathrm{E}-07$ & $1.10 \mathrm{E}-06$ & $1.41 \mathrm{E}-06$ & $1.64 \mathrm{E}-06$ & 1.97E-06 & $7.14 \mathrm{E}-06$ \\
\hline & 192 & $6.78 \mathrm{E}-08$ & $1.67 \mathrm{E}-07$ & $7.06 \mathrm{E}-08$ & $1.28 \mathrm{E}-07$ & $6.06 \mathrm{E}-08$ & $3.83 \mathrm{E}-08$ & 7.61E-08 \\
\hline & 193 & $1.37 \mathrm{E}-07$ & $1.82 \mathrm{E}-07$ & $1.38 \mathrm{E}-07$ & $2.33 \mathrm{E}-09$ & $1.36 \mathrm{E}-07$ & $1.78 \mathrm{E}-07$ & 5.79E-07 \\
\hline & 194 & $3.20 \mathrm{E}-08$ & $6.30 \mathrm{E}-08$ & $3.53 E-08$ & $3.03 E-08$ & $4.06 \mathrm{E}-08$ & $4.48 \mathrm{E}-08$ & $4.44 \mathrm{E}-08$ \\
\hline & 195 & $1.32 \mathrm{E}-08$ & 3.57E-08 & $1.32 \mathrm{E}-08$ & $-1.50 \mathrm{E}-08$ & $-1.15 \mathrm{E}-08$ & $-1.56 \mathrm{E}-08$ & $1.41 \mathrm{E}-08$ \\
\hline & 196 & 8.79E-08 & $1.11 \mathrm{E}-07$ & $9.27 \mathrm{E}-08$ & $1.10 \mathrm{E}-07$ & $1.24 \mathrm{E}-07$ & $1.51 \mathrm{E}-07$ & $1.36 \mathrm{E}-07$ \\
\hline & 197 & 7.39E-09 & $1.77 \mathrm{E}-08$ & $1.13 \mathrm{E}-08$ & $-4.56 \mathrm{E}-09$ & $2.68 \mathrm{E}-08$ & $-2.11 \mathrm{E}-09$ & 8.13E-09 \\
\hline & 198 & $7.23 \mathrm{E}-08$ & $6.55 \mathrm{E}-08$ & $6.84 \mathrm{E}-08$ & $-1.49 E-07$ & $-5.72 E-08$ & $-3.39 E-08$ & $8.26 \mathrm{E}-08$ \\
\hline & 199 & $1.70 \mathrm{E}-08$ & $8.21 \mathrm{E}-08$ & $1.94 \mathrm{E}-08$ & $1.01 \mathrm{E}-08$ & $1.68 \mathrm{E}-08$ & $2.83 \mathrm{E}-08$ & $1.81 \mathrm{E}-08$ \\
\hline & 200 & 4.44E-09 & $2.22 \mathrm{E}-08$ & 6.33E-09 & 2.75E-09 & $6.45 \mathrm{E}-09$ & $-1.38 \mathrm{E}-08$ & 4.59E-09 \\
\hline & 201 & $7.06 \mathrm{E}-07$ & $3.68 \mathrm{E}-07$ & $7.35 \mathrm{E}-07$ & $9.21 \mathrm{E}-07$ & $1.06 \mathrm{E}-06$ & $1.18 \mathrm{E}-06$ & $1.14 \mathrm{E}-06$ \\
\hline & 202 & $4.55 \mathrm{E}-07$ & 7.77E-07 & $5.72 \mathrm{E}-07$ & $5.82 \mathrm{E}-07$ & $6.40 \mathrm{E}-07$ & $6.73 \mathrm{E}-07$ & $6.31 \mathrm{E}-07$ \\
\hline & 203 & $1.01 \mathrm{E}-07$ & $6.33 \mathrm{E}-07$ & $1.11 \mathrm{E}-07$ & $5.29 \mathrm{E}-08$ & $2.62 \mathrm{E}-08$ & $1.35 \mathrm{E}-08$ & $0.00 E+00$ \\
\hline & 204 & $2.30 \mathrm{E}-07$ & $3.28 \mathrm{E}-07$ & $2.25 \mathrm{E}-07$ & $2.26 \mathrm{E}-07$ & $2.56 \mathrm{E}-07$ & $3.07 \mathrm{E}-07$ & $2.82 \mathrm{E}-07$ \\
\hline & 205 & 4.69E-07 & $1.01 \mathrm{E}-06$ & 4.67E-07 & $4.28 \mathrm{E}-07$ & 4.64E-07 & $5.50 \mathrm{E}-07$ & $5.01 \mathrm{E}-07$ \\
\hline & 206 & $1.04 \mathrm{E}-05$ & 4.10E-06 & $2.38 \mathrm{E}-06$ & $1.81 \mathrm{E}-06$ & $4.90 \mathrm{E}-07$ & $2.65 \mathrm{E}-05$ & $3.55 \mathrm{E}-06$ \\
\hline & 207 & $2.92 \mathrm{E}-07$ & $1.14 \mathrm{E}-06$ & 3.10E-07 & $2.05 \mathrm{E}-07$ & $1.76 \mathrm{E}-07$ & $1.86 \mathrm{E}-07$ & $1.54 \mathrm{E}-07$ \\
\hline & 208 & $1.64 \mathrm{E}-06$ & $1.91 \mathrm{E}-06$ & 1.67E-06 & $2.52 \mathrm{E}-06$ & $6.33 \mathrm{E}-05$ & $1.91 \mathrm{E}-06$ & $2.14 \mathrm{E}-06$ \\
\hline & 209 & $3.38 \mathrm{E}-06$ & 3.16E-06 & $3.58 \mathrm{E}-06$ & $5.02 \mathrm{E}-06$ & $1.15 \mathrm{E}-04$ & 5.57E-06 & $9.30 \mathrm{E}-06$ \\
\hline & 210 & $1.05 \mathrm{E}-05$ & $5.86 \mathrm{E}-06$ & $1.15 \mathrm{E}-05$ & $1.58 \mathrm{E}-05$ & $1.95 \mathrm{E}-04$ & $2.26 \mathrm{E}-05$ & 4.41E-05 \\
\hline & 211 & $3.30 \mathrm{E}-07$ & $2.03 \mathrm{E}-06$ & $3.73 E-07$ & $2.14 \mathrm{E}-07$ & 4.85E-05 & $1.34 \mathrm{E}-07$ & $1.92 \mathrm{E}-07$ \\
\hline & 212 & $2.08 \mathrm{E}-06$ & $2.62 \mathrm{E}-06$ & $2.05 \mathrm{E}-06$ & $2.31 \mathrm{E}-06$ & $1.60 \mathrm{E}-06$ & 7.10E-06 & $2.81 \mathrm{E}-06$ \\
\hline & 213 & $6.50 \mathrm{E}-06$ & $6.15 \mathrm{E}-06$ & $6.36 \mathrm{E}-06$ & $7.16 \mathrm{E}-06$ & $7.23 \mathrm{E}-06$ & $9.71 \mathrm{E}-06$ & $8.52 \mathrm{E}-06$ \\
\hline & 214 & $2.30 \mathrm{E}-05$ & $1.55 \mathrm{E}-05$ & $2.24 \mathrm{E}-05$ & $2.56 \mathrm{E}-05$ & $2.78 \mathrm{E}-05$ & 2.67E-05 & $3.06 \mathrm{E}-05$ \\
\hline & 215 & $9.91 \mathrm{E}-07$ & $3.88 \mathrm{E}-06$ & $1.04 \mathrm{E}-06$ & $7.96 \mathrm{E}-07$ & $8.18 \mathrm{E}-07$ & $-9.51 E-07$ & $6.91 \mathrm{E}-07$ \\
\hline & 216 & $1.35 \mathrm{E}-06$ & $2.30 \mathrm{E}-06$ & $1.41 \mathrm{E}-06$ & $1.48 \mathrm{E}-06$ & $1.71 \mathrm{E}-06$ & 7.70E-06 & $1.58 \mathrm{E}-06$ \\
\hline & 217 & $1.10 \mathrm{E}-06$ & $1.53 \mathrm{E}-06$ & $1.13 \mathrm{E}-06$ & $1.22 \mathrm{E}-06$ & $1.35 \mathrm{E}-06$ & $5.14 \mathrm{E}-06$ & $1.36 \mathrm{E}-06$ \\
\hline & 218 & $5.08 \mathrm{E}-06$ & $4.15 \mathrm{E}-06$ & $5.29 \mathrm{E}-06$ & 5.91E-06 & 7.19E-06 & $3.50 \mathrm{E}-05$ & $6.42 \mathrm{E}-06$ \\
\hline & 219 & $9.79 \mathrm{E}-07$ & $2.12 \mathrm{E}-06$ & $1.03 \mathrm{E}-06$ & $1.03 \mathrm{E}-06$ & 1.17E-06 & 5.19E-06 & 1.07E-06 \\
\hline & 220 & $8.74 \mathrm{E}-07$ & $5.64 \mathrm{E}-07$ & 8.70E-07 & $1.46 \mathrm{E}-06$ & $2.04 \mathrm{E}-06$ & $2.51 \mathrm{E}-06$ & $2.90 \mathrm{E}-06$ \\
\hline & 221 & $4.53 \mathrm{E}-08$ & $3.11 \mathrm{E}-07$ & $5.03 E-08$ & $2.33 \mathrm{E}-08$ & $1.11 \mathrm{E}-08$ & $5.66 \mathrm{E}-09$ & $0.00 E+00$ \\
\hline & 222 & 1.79E-07 & $2.03 \mathrm{E}-07$ & $1.90 \mathrm{E}-07$ & $2.31 \mathrm{E}-07$ & 2.37E-07 & $2.40 \mathrm{E}-07$ & 3.59E-07 \\
\hline & 223 & $3.61 \mathrm{E}-07$ & $6.62 \mathrm{E}-07$ & $3.86 \mathrm{E}-07$ & $4.30 \mathrm{E}-07$ & 4.29E-07 & 4.29E-07 & $6.32 \mathrm{E}-07$ \\
\hline & 224 & 7.41E-06 & 4.54E-06 & $4.68 \mathrm{E}-06$ & 4.67E-06 & 1.39E-05 & $1.80 \mathrm{E}-05$ & $6.54 \mathrm{E}-06$ \\
\hline & 225 & 1.79E-07 & $6.73 \mathrm{E}-07$ & $1.96 \mathrm{E}-07$ & $1.50 \mathrm{E}-07$ & $1.27 \mathrm{E}-07$ & $1.17 \mathrm{E}-07$ & 1.79E-07 \\
\hline & 226 & 8.19E-07 & $9.66 \mathrm{E}-07$ & $8.28 \mathrm{E}-07$ & $1.39 \mathrm{E}-06$ & 8.70E-07 & $1.06 \mathrm{E}-06$ & $1.22 \mathrm{E}-06$ \\
\hline & 227 & $2.00 \mathrm{E}-06$ & 1.69E-06 & 2.01E-06 & $3.14 \mathrm{E}-06$ & $3.18 \mathrm{E}-06$ & $3.94 \mathrm{E}-06$ & $5.68 \mathrm{E}-06$ \\
\hline & 228 & $7.15 \mathrm{E}-06$ & $3.29 \mathrm{E}-06$ & 7.07E-06 & $1.10 \mathrm{E}-05$ & $1.45 \mathrm{E}-05$ & $1.81 \mathrm{E}-05$ & $2.85 \mathrm{E}-05$ \\
\hline & 229 & $2.00 \mathrm{E}-07$ & $1.16 \mathrm{E}-06$ & $2.24 \mathrm{E}-07$ & $1.27 \mathrm{E}-07$ & $1.04 \mathrm{E}-07$ & $1.01 \mathrm{E}-07$ & $1.28 \mathrm{E}-07$ \\
\hline & 230 & $1.00 \mathrm{E}-06$ & $1.43 \mathrm{E}-06$ & $1.00 \mathrm{E}-06$ & $1.10 \mathrm{E}-06$ & $1.30 \mathrm{E}-06$ & $1.38 \mathrm{E}-06$ & $1.46 \mathrm{E}-06$ \\
\hline & 231 & $2.88 \mathrm{E}-06$ & $3.06 \mathrm{E}-06$ & $2.86 \mathrm{E}-06$ & $3.25 \mathrm{E}-06$ & 3.65E-06 & $3.87 \mathrm{E}-06$ & $3.96 \mathrm{E}-06$ \\
\hline & 232 & $9.61 \mathrm{E}-06$ & 7.01E-06 & $9.49 \mathrm{E}-06$ & $1.12 \mathrm{E}-05$ & $1.24 \mathrm{E}-05$ & $1.32 \mathrm{E}-05$ & $1.34 \mathrm{E}-05$ \\
\hline & 233 & $4.70 \mathrm{E}-07$ & $2.24 \mathrm{E}-06$ & 5.03E-07 & $3.46 \mathrm{E}-07$ & $2.96 \mathrm{E}-07$ & 2.79E-07 & $2.63 \mathrm{E}-07$ \\
\hline & 234 & 8.03E-07 & $1.20 \mathrm{E}-06$ & $8.31 \mathrm{E}-07$ & $1.18 \mathrm{E}-06$ & $9.15 \mathrm{E}-07$ & $6.98 \mathrm{E}-07$ & $1.03 \mathrm{E}-06$ \\
\hline & 235 & $9.72 \mathrm{E}-07$ & $1.72 \mathrm{E}-06$ & $9.92 \mathrm{E}-07$ & $1.04 \mathrm{E}-06$ & $1.02 \mathrm{E}-06$ & $8.42 \mathrm{E}-07$ & $1.12 \mathrm{E}-06$ \\
\hline & 236 & $9.02 \mathrm{E}-08$ & $2.82 \mathrm{E}-07$ & $9.64 \mathrm{E}-08$ & $2.16 \mathrm{E}-07$ & $-1.12 \mathrm{E}-07$ & $4.31 \mathrm{E}-07$ & $1.02 \mathrm{E}-07$ \\
\hline
\end{tabular}


Table 4 (continued)

\begin{tabular}{|c|c|c|c|c|c|c|c|c|}
\hline \multirow{2}{*}{$\mathrm{i}$} & $\mathrm{j}$ & $2944.1 \mathrm{eV}$ & $4868.3 \mathrm{eV}$ & $7564.6 \mathrm{eV}$ & $11040 \mathrm{eV}$ & $15221 \mathrm{eV}$ & $65.33 \mathrm{eV}$ & $1714.98 \mathrm{eV}$ \\
\cline { 3 - 9 } & 237 & $1.10 \mathrm{E}-07$ & $2.17 \mathrm{E}-07$ & $1.06 \mathrm{E}-07$ & $1.98 \mathrm{E}-07$ & $2.64 \mathrm{E}-07$ & $-1.82 \mathrm{E}-07$ & $1.26 \mathrm{E}-07$ \\
& 238 & $3.33 \mathrm{E}-06$ & $2.88 \mathrm{E}-06$ & $3.32 \mathrm{E}-06$ & $3.03 \mathrm{E}-06$ & $3.73 \mathrm{E}-06$ & $3.23 \mathrm{E}-06$ & $4.05 \mathrm{E}-06$ \\
& 239 & $2.79 \mathrm{E}-07$ & $3.93 \mathrm{E}-07$ & $3.18 \mathrm{E}-07$ & $9.14 \mathrm{E}-07$ & $-1.27 \mathrm{E}-06$ & $3.08 \mathrm{E}-06$ & $3.70 \mathrm{E}-07$ \\
& 240 & $7.78 \mathrm{E}-08$ & $2.73 \mathrm{E}-07$ & $8.38 \mathrm{E}-08$ & $1.81 \mathrm{E}-07$ & $-9.46 \mathrm{E}-08$ & $3.60 \mathrm{E}-07$ & $8.47 \mathrm{E}-08$ \\
& 241 & $6.50 \mathrm{E}-07$ & $1.67 \mathrm{E}-06$ & $6.78 \mathrm{E}-07$ & $6.46 \mathrm{E}-07$ & $6.27 \mathrm{E}-07$ & $4.97 \mathrm{E}-07$ & $6.57 \mathrm{E}-07$ \\
\hline
\end{tabular}


Table 5. The effective collision strengths at electron plasma temperatures $(650,850,1050,1250,1450,1650,1850) \mathrm{eV}$ for Ne-like Ge ions.

\begin{tabular}{|c|c|c|c|c|c|c|c|c|}
\hline \multirow[b]{2}{*}{1} & \multirow[b]{2}{*}{$\mathrm{J}$} & \multicolumn{7}{|c|}{ Effective collision strengths at temperatures } \\
\hline & & $650 \mathrm{eV}$ & $850 \mathrm{eV}$ & $1050 \mathrm{eV}$ & $1250 \mathrm{eV}$ & $1450 \mathrm{eV}$ & $1650 \mathrm{eV}$ & $1850 \mathrm{eV}$ \\
\hline \multirow[t]{68}{*}{1} & 2 & $2.82 \mathrm{E}-04$ & $3.10 \mathrm{E}-04$ & $2.74 \mathrm{E}-04$ & $2.84 \mathrm{E}-04$ & $2.96 \mathrm{E}-04$ & $3.46 \mathrm{E}-04$ & $4.02 \mathrm{E}-04$ \\
\hline & 3 & $1.01 \mathrm{E}-02$ & $9.93 \mathrm{E}-03$ & $1.13 \mathrm{E}-02$ & $1.03 \mathrm{E}-02$ & $9.39 \mathrm{E}-03$ & $7.68 \mathrm{E}-03$ & $5.74 \mathrm{E}-03$ \\
\hline & 4 & $5.73 E-05$ & $6.30 \mathrm{E}-05$ & $5.55 \mathrm{E}-05$ & $5.76 \mathrm{E}-05$ & $6.01 \mathrm{E}-05$ & 7.03E-05 & $8.15 \mathrm{E}-05$ \\
\hline & 5 & $6.71 \mathrm{E}-03$ & $6.62 \mathrm{E}-03$ & 7.47E-03 & $6.88 \mathrm{E}-03$ & $6.26 \mathrm{E}-03$ & $5.13 \mathrm{E}-03$ & $3.84 \mathrm{E}-03$ \\
\hline & 6 & $6.75 \mathrm{E}-04$ & 7.40E-04 & $6.48 \mathrm{E}-04$ & $6.72 \mathrm{E}-04$ & 7.00E-04 & $8.23 \mathrm{E}-04$ & $9.58 \mathrm{E}-04$ \\
\hline & 7 & $2.59 \mathrm{E}-03$ & $2.56 \mathrm{E}-03$ & $2.53 \mathrm{E}-03$ & $2.51 \mathrm{E}-03$ & $2.49 \mathrm{E}-03$ & $2.48 \mathrm{E}-03$ & $2.46 \mathrm{E}-03$ \\
\hline & 8 & 7.90E-04 & $8.62 \mathrm{E}-04$ & 7.43E-04 & 7.67E-04 & 7.97E-04 & $9.49 E-04$ & $1.12 \mathrm{E}-03$ \\
\hline & 9 & $3.27 \mathrm{E}-04$ & 3.57E-04 & 3.09E-04 & 3.19E-04 & $3.31 \mathrm{E}-04$ & $3.94 \mathrm{E}-04$ & $4.62 \mathrm{E}-04$ \\
\hline & 10 & $2.40 \mathrm{E}-03$ & 2.35E-03 & $2.34 \mathrm{E}-03$ & $2.31 \mathrm{E}-03$ & 2.29E-03 & $2.25 \mathrm{E}-03$ & $2.21 \mathrm{E}-03$ \\
\hline & 11 & $5.28 \mathrm{E}-03$ & $5.24 \mathrm{E}-03$ & $5.22 \mathrm{E}-03$ & $5.22 \mathrm{E}-03$ & $5.22 \mathrm{E}-03$ & $5.20 \mathrm{E}-03$ & $5.18 \mathrm{E}-03$ \\
\hline & 12 & $3.21 \mathrm{E}-04$ & $3.50 \mathrm{E}-04$ & $3.02 \mathrm{E}-04$ & $3.12 \mathrm{E}-04$ & $3.24 \mathrm{E}-04$ & $3.85 E-04$ & 4.53E-04 \\
\hline & 13 & $3.63 \mathrm{E}-04$ & 3.97E-04 & $3.45 \mathrm{E}-04$ & $3.56 \mathrm{E}-04$ & $3.71 \mathrm{E}-04$ & $4.39 \mathrm{E}-04$ & $5.14 \mathrm{E}-04$ \\
\hline & 14 & $2.56 \mathrm{E}-03$ & $2.52 \mathrm{E}-03$ & $2.50 \mathrm{E}-03$ & 2.47E-03 & $2.45 \mathrm{E}-03$ & $2.42 \mathrm{E}-03$ & $2.40 \mathrm{E}-03$ \\
\hline & 15 & 2.67E-02 & $2.65 \mathrm{E}-02$ & $2.65 \mathrm{E}-02$ & $2.64 \mathrm{E}-02$ & $2.64 \mathrm{E}-02$ & $2.62 \mathrm{E}-02$ & $2.61 \mathrm{E}-02$ \\
\hline & 16 & $3.53 \mathrm{E}-04$ & $3.85 E-04$ & $3.29 \mathrm{E}-04$ & $3.38 \mathrm{E}-04$ & $3.51 \mathrm{E}-04$ & $4.21 \mathrm{E}-04$ & $4.98 \mathrm{E}-04$ \\
\hline & 17 & 1.77E-03 & $1.83 \mathrm{E}-03$ & 1.79E-03 & $1.76 \mathrm{E}-03$ & $1.73 \mathrm{E}-03$ & $1.80 \mathrm{E}-03$ & $1.88 \mathrm{E}-03$ \\
\hline & 18 & $1.16 \mathrm{E}-03$ & $1.26 \mathrm{E}-03$ & 1.07E-03 & $1.10 \mathrm{E}-03$ & $1.14 \mathrm{E}-03$ & 1.37E-03 & $1.63 \mathrm{E}-03$ \\
\hline & 19 & $1.20 \mathrm{E}-03$ & $1.30 \mathrm{E}-03$ & $1.11 \mathrm{E}-03$ & $1.14 \mathrm{E}-03$ & $1.18 \mathrm{E}-03$ & $1.43 \mathrm{E}-03$ & $1.69 \mathrm{E}-03$ \\
\hline & 20 & 2.37E-03 & $2.37 \mathrm{E}-03$ & $2.31 \mathrm{E}-03$ & $2.30 \mathrm{E}-03$ & $2.29 \mathrm{E}-03$ & $2.33 \mathrm{E}-03$ & $2.38 \mathrm{E}-03$ \\
\hline & 21 & $4.53 \mathrm{E}-04$ & $4.89 \mathrm{E}-04$ & $4.08 \mathrm{E}-04$ & $4.18 \mathrm{E}-04$ & $4.32 \mathrm{E}-04$ & $5.28 \mathrm{E}-04$ & $6.33 \mathrm{E}-04$ \\
\hline & 22 & $2.05 \mathrm{E}-03$ & 2.03E-03 & 2.01E-03 & $1.99 \mathrm{E}-03$ & 1.97E-03 & 1.97E-03 & $1.96 \mathrm{E}-03$ \\
\hline & 23 & $1.11 \mathrm{E}-01$ & $1.10 \mathrm{E}-01$ & $1.20 \mathrm{E}-01$ & $1.13 \mathrm{E}-01$ & $1.05 \mathrm{E}-01$ & $8.98 \mathrm{E}-02$ & $7.25 \mathrm{E}-02$ \\
\hline & 24 & $5.53 \mathrm{E}-04$ & 5.99E-04 & $5.05 \mathrm{E}-04$ & $5.18 \mathrm{E}-04$ & $5.36 \mathrm{E}-04$ & $6.50 \mathrm{E}-04$ & 7.75E-04 \\
\hline & 25 & $8.13 E-04$ & 8.83E-04 & 7.48E-04 & 7.69E-04 & $7.96 \mathrm{E}-04$ & $9.61 \mathrm{E}-04$ & $1.14 \mathrm{E}-03$ \\
\hline & 26 & $2.33 \mathrm{E}-03$ & 2.31E-03 & $2.28 \mathrm{E}-03$ & $2.26 \mathrm{E}-03$ & $2.24 \mathrm{E}-03$ & $2.26 \mathrm{E}-03$ & $2.27 \mathrm{E}-03$ \\
\hline & 27 & $1.90 \mathrm{E}-01$ & $1.87 \mathrm{E}-01$ & $2.05 \mathrm{E}-01$ & $1.93 \mathrm{E}-01$ & $1.79 \mathrm{E}-01$ & $1.53 \mathrm{E}-01$ & $1.23 \mathrm{E}-01$ \\
\hline & 28 & $1.83 \mathrm{E}-04$ & $2.00 \mathrm{E}-04$ & $1.72 \mathrm{E}-04$ & 1.77E-04 & $1.84 \mathrm{E}-04$ & $2.20 \mathrm{E}-04$ & $2.59 \mathrm{E}-04$ \\
\hline & 29 & $1.22 \mathrm{E}-02$ & $1.21 \mathrm{E}-02$ & $1.21 \mathrm{E}-02$ & $1.21 \mathrm{E}-02$ & $1.21 \mathrm{E}-02$ & $1.19 \mathrm{E}-02$ & $1.18 \mathrm{E}-02$ \\
\hline & 30 & $4.84 \mathrm{E}-05$ & 5.31E-05 & 4.66E-05 & 4.83E-05 & 5.03E-05 & $5.90 \mathrm{E}-05$ & $6.86 \mathrm{E}-05$ \\
\hline & 31 & 4.10E-03 & 4.06E-03 & $4.56 \mathrm{E}-03$ & $4.22 \mathrm{E}-03$ & $3.83 \mathrm{E}-03$ & $3.14 \mathrm{E}-03$ & $2.34 \mathrm{E}-03$ \\
\hline & 32 & $2.31 \mathrm{E}-04$ & $2.53 \mathrm{E}-04$ & $2.23 \mathrm{E}-04$ & 2.31E-04 & $2.41 \mathrm{E}-04$ & $2.83 \mathrm{E}-04$ & $3.28 \mathrm{E}-04$ \\
\hline & 33 & $1.72 \mathrm{E}-02$ & $1.71 \mathrm{E}-02$ & $1.97 \mathrm{E}-02$ & 1.77E-02 & $1.60 \mathrm{E}-02$ & $1.29 \mathrm{E}-02$ & $9.31 \mathrm{E}-03$ \\
\hline & 34 & 3.31E-04 & $3.61 \mathrm{E}-04$ & $3.11 \mathrm{E}-04$ & $3.21 \mathrm{E}-04$ & $3.34 \mathrm{E}-04$ & $3.98 \mathrm{E}-04$ & $4.68 \mathrm{E}-04$ \\
\hline & 35 & 7.47E-04 & 7.83E-04 & 7.18E-04 & 7.31E-04 & 7.49E-04 & $8.45 E-04$ & $9.51 \mathrm{E}-04$ \\
\hline & 36 & 7.64E-04 & 8.34E-04 & 7.19E-04 & $7.42 \mathrm{E}-04$ & 7.70E-04 & $9.18 \mathrm{E}-04$ & $1.08 \mathrm{E}-03$ \\
\hline & 37 & $2.38 \mathrm{E}-02$ & $2.30 \mathrm{E}-02$ & 2.33E-02 & $2.29 \mathrm{E}-02$ & $2.25 \mathrm{E}-02$ & $2.15 \mathrm{E}-02$ & $2.04 \mathrm{E}-02$ \\
\hline & 38 & $9.12 \mathrm{E}-05$ & $1.00 \mathrm{E}-04$ & 8.87E-05 & $9.21 \mathrm{E}-05$ & $9.61 \mathrm{E}-05$ & $1.12 \mathrm{E}-04$ & $1.30 \mathrm{E}-04$ \\
\hline & 39 & $1.38 \mathrm{E}-03$ & $1.36 \mathrm{E}-03$ & $1.52 \mathrm{E}-03$ & $1.41 \mathrm{E}-03$ & $1.28 \mathrm{E}-03$ & $1.06 \mathrm{E}-03$ & 8.01E-04 \\
\hline & 40 & $1.81 \mathrm{E}-04$ & $1.98 \mathrm{E}-04$ & $1.74 \mathrm{E}-04$ & $1.80 \mathrm{E}-04$ & $1.87 \mathrm{E}-04$ & $2.20 \mathrm{E}-04$ & $2.56 \mathrm{E}-04$ \\
\hline & 41 & 5.47E-04 & $5.46 \mathrm{E}-04$ & $5.34 \mathrm{E}-04$ & $5.30 \mathrm{E}-04$ & $5.28 \mathrm{E}-04$ & $5.36 \mathrm{E}-04$ & $5.46 \mathrm{E}-04$ \\
\hline & 42 & $2.71 \mathrm{E}-04$ & $2.96 \mathrm{E}-04$ & $2.56 \mathrm{E}-04$ & $2.64 \mathrm{E}-04$ & $2.75 \mathrm{E}-04$ & $3.26 \mathrm{E}-04$ & $3.83 E-04$ \\
\hline & 43 & $1.11 \mathrm{E}-04$ & $1.21 \mathrm{E}-04$ & $1.05 \mathrm{E}-04$ & $1.09 \mathrm{E}-04$ & $1.13 \mathrm{E}-04$ & $1.34 \mathrm{E}-04$ & 1.57E-04 \\
\hline & 44 & 4.76E-04 & $4.72 \mathrm{E}-04$ & 4.64E-04 & $4.59 \mathrm{E}-04$ & $4.56 \mathrm{E}-04$ & $4.58 \mathrm{E}-04$ & $4.61 \mathrm{E}-04$ \\
\hline & 45 & $4.31 \mathrm{E}-03$ & 4.27E-03 & 4.27E-03 & 4.26E-03 & 4.25E-03 & 4.22E-03 & 4.19E-03 \\
\hline & 46 & $1.89 \mathrm{E}-05$ & 2.07E-05 & $1.83 \mathrm{E}-05$ & $1.89 \mathrm{E}-05$ & $1.98 \mathrm{E}-05$ & 2.31E-05 & $2.68 \mathrm{E}-05$ \\
\hline & 47 & $1.18 \mathrm{E}-03$ & 1.17E-03 & $1.30 \mathrm{E}-03$ & $1.21 \mathrm{E}-03$ & $1.11 \mathrm{E}-03$ & $9.17 \mathrm{E}-04$ & 7.01E-04 \\
\hline & 48 & 1.19E-04 & $1.30 \mathrm{E}-04$ & $1.11 \mathrm{E}-04$ & $1.15 \mathrm{E}-04$ & 1.19E-04 & $1.42 \mathrm{E}-04$ & $1.68 \mathrm{E}-04$ \\
\hline & 49 & 4.74E-04 & 4.95E-04 & $4.74 \mathrm{E}-04$ & 4.71E-04 & 4.70E-04 & $5.06 \mathrm{E}-04$ & $5.44 \mathrm{E}-04$ \\
\hline & 50 & $4.04 \mathrm{E}-04$ & 4.39E-04 & $3.73 E-04$ & $3.83 \mathrm{E}-04$ & 3.97E-04 & 4.79E-04 & $5.69 \mathrm{E}-04$ \\
\hline & 51 & $5.56 \mathrm{E}-04$ & 5.59E-04 & $5.40 \mathrm{E}-04$ & $5.36 \mathrm{E}-04$ & $5.33 \mathrm{E}-04$ & $5.54 \mathrm{E}-04$ & $5.76 \mathrm{E}-04$ \\
\hline & 52 & $3.62 \mathrm{E}-04$ & 3.93E-04 & $3.35 \mathrm{E}-04$ & $3.45 \mathrm{E}-04$ & 3.57E-04 & $4.30 \mathrm{E}-04$ & 5.09E-04 \\
\hline & 53 & $1.72 \mathrm{E}-04$ & $1.86 \mathrm{E}-04$ & $1.56 \mathrm{E}-04$ & $1.60 \mathrm{E}-04$ & $1.65 \mathrm{E}-04$ & $2.02 \mathrm{E}-04$ & $2.41 \mathrm{E}-04$ \\
\hline & 54 & $4.34 \mathrm{E}-04$ & 4.33E-04 & $4.20 \mathrm{E}-04$ & 4.15E-04 & 4.11E-04 & $4.22 \mathrm{E}-04$ & $4.35 \mathrm{E}-04$ \\
\hline & 55 & $3.52 \mathrm{E}-02$ & $3.47 \mathrm{E}-02$ & $3.78 \mathrm{E}-02$ & $3.57 \mathrm{E}-02$ & $3.33 \mathrm{E}-02$ & $2.88 \mathrm{E}-02$ & $2.36 \mathrm{E}-02$ \\
\hline & 56 & $1.15 \mathrm{E}-04$ & $1.26 \mathrm{E}-04$ & 1.09E-04 & $1.12 \mathrm{E}-04$ & 1.17E-04 & $1.39 \mathrm{E}-04$ & $1.63 \mathrm{E}-04$ \\
\hline & 57 & $1.31 \mathrm{E}-04$ & $1.43 \mathrm{E}-04$ & $1.25 \mathrm{E}-04$ & $1.29 \mathrm{E}-04$ & $1.34 \mathrm{E}-04$ & $1.59 \mathrm{E}-04$ & $1.86 \mathrm{E}-04$ \\
\hline & 58 & 5.67E-04 & $5.64 \mathrm{E}-04$ & $5.53 \mathrm{E}-04$ & $5.48 \mathrm{E}-04$ & $5.45 \mathrm{E}-04$ & 5.51E-04 & 5.59E-04 \\
\hline & 59 & $5.65 \mathrm{E}-05$ & $6.09 \mathrm{E}-05$ & $5.05 \mathrm{E}-05$ & $5.16 \mathrm{E}-05$ & $5.32 \mathrm{E}-05$ & $6.55 \mathrm{E}-05$ & $7.88 \mathrm{E}-05$ \\
\hline & 60 & $2.11 \mathrm{E}-04$ & 2.09E-04 & $2.03 \mathrm{E}-04$ & $2.01 \mathrm{E}-04$ & $2.00 \mathrm{E}-04$ & $2.03 E-04$ & $2.06 \mathrm{E}-04$ \\
\hline & 61 & $4.85 \mathrm{E}-04$ & 4.73E-04 & $4.72 \mathrm{E}-04$ & $4.62 \mathrm{E}-04$ & $4.54 \mathrm{E}-04$ & $4.43 E-04$ & 4.31E-04 \\
\hline & 62 & 8.99E-05 & $9.66 \mathrm{E}-05$ & 7.96E-05 & $8.11 \mathrm{E}-05$ & $8.36 \mathrm{E}-05$ & $1.03 E-04$ & $1.25 \mathrm{E}-04$ \\
\hline & 63 & $6.92 \mathrm{E}-05$ & 7.45E-05 & $6.14 \mathrm{E}-05$ & $6.26 \mathrm{E}-05$ & $6.45 \mathrm{E}-05$ & $7.98 \mathrm{E}-05$ & $9.65 \mathrm{E}-05$ \\
\hline & 64 & $2.20 \mathrm{E}-03$ & $2.11 \mathrm{E}-03$ & $2.16 \mathrm{E}-03$ & $2.10 \mathrm{E}-03$ & $2.05 E-03$ & $1.92 \mathrm{E}-03$ & 1.77E-03 \\
\hline & 65 & $4.54 \mathrm{E}-05$ & $4.88 \mathrm{E}-05$ & 3.99E-05 & $4.06 \mathrm{E}-05$ & 4.18E-05 & $5.20 \mathrm{E}-05$ & $6.32 \mathrm{E}-05$ \\
\hline & 66 & $9.28 \mathrm{E}-05$ & 9.33E-05 & $8.78 \mathrm{E}-05$ & $8.72 \mathrm{E}-05$ & $8.72 \mathrm{E}-05$ & $9.32 \mathrm{E}-05$ & $9.98 \mathrm{E}-05$ \\
\hline & 67 & $4.14 \mathrm{E}-03$ & 4.10E-03 & 4.09E-03 & 4.09E-03 & 4.08E-03 & 4.05E-03 & $4.02 \mathrm{E}-03$ \\
\hline & 68 & $2.04 \mathrm{E}-04$ & $2.22 \mathrm{E}-04$ & $1.88 \mathrm{E}-04$ & $1.93 \mathrm{E}-04$ & $2.00 \mathrm{E}-04$ & $2.42 \mathrm{E}-04$ & 2.87E-04 \\
\hline & 69 & 3.27E-04 & 3.55E-04 & 3.02E-04 & 3.11E-04 & $3.22 \mathrm{E}-04$ & $3.88 \mathrm{E}-04$ & 4.59E-04 \\
\hline
\end{tabular}


Table 5 (continued)

\begin{tabular}{|c|c|c|c|c|c|c|c|c|}
\hline \multirow[b]{2}{*}{ I } & \multirow[b]{2}{*}{$\mathrm{j}$} & \multicolumn{7}{|c|}{ Effective collision strengths at temperatures } \\
\hline & & $650 \mathrm{eV}$ & $850 \mathrm{eV}$ & $1050 \mathrm{eV}$ & $1250 \mathrm{eV}$ & $1450 \mathrm{eV}$ & $1650 \mathrm{eV}$ & $1850 \mathrm{eV}$ \\
\hline & 70 & $5.11 \mathrm{E}-04$ & $5.13 \mathrm{E}-04$ & $4.95 \mathrm{E}-04$ & $4.91 \mathrm{E}-04$ & $4.88 \mathrm{E}-04$ & $5.06 \mathrm{E}-04$ & $5.25 \mathrm{E}-04$ \\
\hline & 71 & $2.65 \mathrm{E}-02$ & $2.61 \mathrm{E}-02$ & $2.85 \mathrm{E}-02$ & $2.69 \mathrm{E}-02$ & $2.51 \mathrm{E}-02$ & $2.17 \mathrm{E}-02$ & 1.77E-02 \\
\hline & 72 & $4.75 \mathrm{E}-05$ & $5.10 \mathrm{E}-05$ & 4.20E-05 & $4.27 \mathrm{E}-05$ & $4.40 \mathrm{E}-05$ & $5.46 \mathrm{E}-05$ & $6.61 \mathrm{E}-05$ \\
\hline & 73 & $1.43 \mathrm{E}-04$ & $1.43 \mathrm{E}-04$ & $1.37 \mathrm{E}-04$ & $1.36 \mathrm{E}-04$ & $1.35 \mathrm{E}-04$ & $1.40 \mathrm{E}-04$ & $1.46 \mathrm{E}-04$ \\
\hline & 74 & $1.23 \mathrm{E}-03$ & $1.19 \mathrm{E}-03$ & $1.21 \mathrm{E}-03$ & $1.18 \mathrm{E}-03$ & $1.15 \mathrm{E}-03$ & $1.08 \mathrm{E}-03$ & $1.01 \mathrm{E}-03$ \\
\hline & 75 & $8.18 \mathrm{E}-05$ & 8.81E-05 & $7.29 \mathrm{E}-05$ & 7.44E-05 & 7.67E-05 & $9.45 \mathrm{E}-05$ & $1.14 \mathrm{E}-04$ \\
\hline & 76 & 4.76E-05 & 5.25E-05 & 4.65E-05 & 4.83E-05 & $5.04 \mathrm{E}-05$ & $5.87 \mathrm{E}-05$ & 6.79E-05 \\
\hline & 77 & $5.87 \mathrm{E}-04$ & $5.78 \mathrm{E}-04$ & $6.48 \mathrm{E}-04$ & $5.98 \mathrm{E}-04$ & $5.45 \mathrm{E}-04$ & 4.49E-04 & 3.39E-04 \\
\hline & 78 & $1.12 \mathrm{E}-04$ & 1.23E-04 & $1.06 \mathrm{E}-04$ & $1.10 \mathrm{E}-04$ & $1.14 \mathrm{E}-04$ & $1.36 \mathrm{E}-04$ & 1.59E-04 \\
\hline & 79 & $1.89 \mathrm{E}-03$ & $1.86 \mathrm{E}-03$ & $1.87 \mathrm{E}-03$ & $1.86 \mathrm{E}-03$ & $1.86 \mathrm{E}-03$ & $1.83 \mathrm{E}-03$ & $1.80 \mathrm{E}-03$ \\
\hline & 80 & 2.67E-04 & 2.66E-04 & 2.61E-04 & $2.59 \mathrm{E}-04$ & $2.58 \mathrm{E}-04$ & 2.61E-04 & $2.65 \mathrm{E}-04$ \\
\hline & 81 & $4.86 \mathrm{E}-05$ & 5.32E-05 & 4.65E-05 & $4.82 \mathrm{E}-05$ & 5.01E-05 & 5.91E-05 & $6.89 E-05$ \\
\hline & 82 & $1.28 \mathrm{E}-04$ & 1.40E-04 & $1.21 \mathrm{E}-04$ & $1.25 \mathrm{E}-04$ & 1.30E-04 & $1.54 \mathrm{E}-04$ & $1.81 \mathrm{E}-04$ \\
\hline & 83 & $4.75 \mathrm{E}-05$ & $5.20 \mathrm{E}-05$ & 4.51E-05 & 4.67E-05 & 4.86E-05 & $5.75 E-05$ & $6.73 E-05$ \\
\hline & 84 & $2.29 \mathrm{E}-04$ & 2.27E-04 & $2.24 \mathrm{E}-04$ & $2.21 \mathrm{E}-04$ & $2.20 \mathrm{E}-04$ & $2.21 \mathrm{E}-04$ & $2.22 \mathrm{E}-04$ \\
\hline & 85 & $2.56 \mathrm{E}-03$ & $2.53 \mathrm{E}-03$ & $2.53 \mathrm{E}-03$ & $2.52 \mathrm{E}-03$ & $2.52 \mathrm{E}-03$ & $2.49 \mathrm{E}-03$ & $2.47 \mathrm{E}-03$ \\
\hline & 86 & $3.94 \mathrm{E}-05$ & 4.29E-05 & $3.68 \mathrm{E}-05$ & 3.79E-05 & 3.93E-05 & 4.71E-05 & 5.55E-05 \\
\hline & 87 & $5.90 \mathrm{E}-04$ & $5.90 \mathrm{E}-04$ & $6.38 \mathrm{E}-04$ & $6.01 \mathrm{E}-04$ & 5.57E-04 & $4.90 \mathrm{E}-04$ & $4.12 \mathrm{E}-04$ \\
\hline & 88 & $3.58 \mathrm{E}-05$ & $3.92 \mathrm{E}-05$ & $3.41 \mathrm{E}-05$ & $3.52 \mathrm{E}-05$ & 3.67E-05 & 4.34E-05 & $5.08 \mathrm{E}-05$ \\
\hline & 89 & $1.46 \mathrm{E}-03$ & $1.45 \mathrm{E}-03$ & $1.59 \mathrm{E}-03$ & $1.49 \mathrm{E}-03$ & $1.38 \mathrm{E}-03$ & 1.17E-03 & $9.39 \mathrm{E}-04$ \\
\hline & 90 & $1.84 \mathrm{E}-04$ & $1.99 \mathrm{E}-04$ & 1.70E-04 & $1.74 \mathrm{E}-04$ & $1.81 \mathrm{E}-04$ & $2.18 \mathrm{E}-04$ & $2.58 \mathrm{E}-04$ \\
\hline & 91 & $2.22 \mathrm{E}-04$ & $2.24 \mathrm{E}-04$ & $2.14 \mathrm{E}-04$ & $2.13 \mathrm{E}-04$ & $2.12 \mathrm{E}-04$ & $2.22 \mathrm{E}-04$ & $2.34 \mathrm{E}-04$ \\
\hline & 92 & $1.87 \mathrm{E}-04$ & $2.03 \mathrm{E}-04$ & $1.73 \mathrm{E}-04$ & $1.78 \mathrm{E}-04$ & $1.84 \mathrm{E}-04$ & $2.22 \mathrm{E}-04$ & $2.63 \mathrm{E}-04$ \\
\hline & 93 & $7.80 \mathrm{E}-05$ & $8.44 \mathrm{E}-05$ & $7.06 \mathrm{E}-05$ & $7.23 \mathrm{E}-05$ & 7.47E-05 & $9.12 \mathrm{E}-05$ & $1.09 \mathrm{E}-04$ \\
\hline & 94 & $1.70 \mathrm{E}-04$ & 1.70E-04 & $1.63 \mathrm{E}-04$ & $1.61 \mathrm{E}-04$ & $1.60 \mathrm{E}-04$ & $1.67 \mathrm{E}-04$ & 1.76E-04 \\
\hline & 95 & $7.00 \mathrm{E}-05$ & 7.70E-05 & $6.81 \mathrm{E}-05$ & $7.08 \mathrm{E}-05$ & 7.39E-05 & $8.62 \mathrm{E}-05$ & $9.97 \mathrm{E}-05$ \\
\hline & 96 & $1.44 \mathrm{E}-02$ & $1.42 \mathrm{E}-02$ & $1.58 \mathrm{E}-02$ & $1.48 \mathrm{E}-02$ & $1.36 \mathrm{E}-02$ & $1.13 \mathrm{E}-02$ & $8.80 \mathrm{E}-03$ \\
\hline & 97 & 5.47E-03 & 5.39E-03 & $5.74 \mathrm{E}-03$ & $5.50 \mathrm{E}-03$ & $5.21 \mathrm{E}-03$ & $4.68 \mathrm{E}-03$ & 4.07E-03 \\
\hline & 98 & 5.15E-05 & 5.57E-05 & 4.66E-05 & 4.77E-05 & 4.93E-05 & $6.02 \mathrm{E}-05$ & $7.21 \mathrm{E}-05$ \\
\hline & 99 & $3.18 \mathrm{E}-04$ & $3.13 \mathrm{E}-04$ & $3.08 \mathrm{E}-04$ & $3.03 E-04$ & 2.99E-04 & $2.98 \mathrm{E}-04$ & $2.97 \mathrm{E}-04$ \\
\hline & 100 & $1.18 \mathrm{E}-04$ & 1.17E-04 & $1.13 \mathrm{E}-04$ & $1.12 \mathrm{E}-04$ & 1.11E-04 & $1.14 \mathrm{E}-04$ & 1.17E-04 \\
\hline & 101 & $5.80 \mathrm{E}-05$ & $6.24 \mathrm{E}-05$ & $5.16 \mathrm{E}-05$ & 5.27E-05 & $5.43 \mathrm{E}-05$ & $6.70 \mathrm{E}-05$ & 8.09E-05 \\
\hline & 102 & $6.25 \mathrm{E}-05$ & $6.75 \mathrm{E}-05$ & 5.61E-05 & $5.74 \mathrm{E}-05$ & 5.92E-05 & 7.27E-05 & $8.74 \mathrm{E}-05$ \\
\hline & 103 & $7.90 \mathrm{E}-06$ & $8.48 \mathrm{E}-06$ & $6.95 \mathrm{E}-06$ & 7.07E-06 & $7.28 \mathrm{E}-06$ & $9.05 E-06$ & $1.10 \mathrm{E}-05$ \\
\hline & 104 & 5.90E-05 & $5.78 \mathrm{E}-05$ & $5.74 \mathrm{E}-05$ & 5.69E-05 & 5.65E-05 & 5.58E-05 & $5.52 \mathrm{E}-05$ \\
\hline & 105 & $2.68 \mathrm{E}-05$ & 2.87E-05 & $2.35 \mathrm{E}-05$ & 2.39E-05 & $2.46 \mathrm{E}-05$ & $3.06 \mathrm{E}-05$ & $3.73 E-05$ \\
\hline & 106 & $1.47 \mathrm{E}-03$ & $1.41 \mathrm{E}-03$ & $1.44 \mathrm{E}-03$ & $1.40 \mathrm{E}-03$ & $1.36 \mathrm{E}-03$ & $1.28 \mathrm{E}-03$ & $1.18 \mathrm{E}-03$ \\
\hline & 107 & 5.69E-05 & $5.74 \mathrm{E}-05$ & 5.36E-05 & $5.32 \mathrm{E}-05$ & 5.32E-05 & $5.75 \mathrm{E}-05$ & $6.22 \mathrm{E}-05$ \\
\hline & 108 & 8.93E-06 & 8.93E-06 & $8.48 \mathrm{E}-06$ & $8.43 E-06$ & $8.42 \mathrm{E}-06$ & 8.90E-06 & $9.41 \mathrm{E}-06$ \\
\hline & 109 & 4.69E-05 & $4.55 \mathrm{E}-05$ & $4.58 \mathrm{E}-05$ & $4.53 \mathrm{E}-05$ & $4.48 \mathrm{E}-05$ & 4.35E-05 & 4.20E-05 \\
\hline & 110 & 4.83E-06 & $5.16 \mathrm{E}-06$ & $4.20 \mathrm{E}-06$ & $4.26 \mathrm{E}-06$ & 4.38E-06 & 5.49E-06 & 6.70E-06 \\
\hline & 111 & $6.75 \mathrm{E}-06$ & $7.25 \mathrm{E}-06$ & 5.93E-06 & $6.03 \mathrm{E}-06$ & $6.21 \mathrm{E}-06$ & $7.72 \mathrm{E}-06$ & $9.38 \mathrm{E}-06$ \\
\hline & 112 & $1.96 \mathrm{E}-06$ & $2.10 \mathrm{E}-06$ & $1.70 \mathrm{E}-06$ & $1.72 \mathrm{E}-06$ & $1.77 \mathrm{E}-06$ & $2.22 \mathrm{E}-06$ & $2.72 \mathrm{E}-06$ \\
\hline & 113 & $3.61 \mathrm{E}-06$ & 3.67E-06 & $3.36 \mathrm{E}-06$ & $3.36 \mathrm{E}-06$ & $3.38 \mathrm{E}-06$ & $3.76 \mathrm{E}-06$ & 4.19E-06 \\
\hline & 114 & 7.11E-06 & $7.82 \mathrm{E}-06$ & $6.91 \mathrm{E}-06$ & 7.17E-06 & $7.48 \mathrm{E}-06$ & $8.74 \mathrm{E}-06$ & $1.01 \mathrm{E}-05$ \\
\hline & 115 & 8.04E-05 & 8.03E-05 & 8.06E-05 & 7.91E-05 & $7.76 \mathrm{E}-05$ & 7.65E-05 & 7.51E-05 \\
\hline & 116 & $5.85 \mathrm{E}-05$ & $6.39 \mathrm{E}-05$ & 5.49E-05 & 5.66E-05 & $5.88 \mathrm{E}-05$ & 7.01E-05 & $8.26 \mathrm{E}-05$ \\
\hline & 117 & $3.47 \mathrm{E}-04$ & 3.46E-04 & $3.38 \mathrm{E}-04$ & $3.36 \mathrm{E}-04$ & $3.35 \mathrm{E}-04$ & $3.41 \mathrm{E}-04$ & $3.47 \mathrm{E}-04$ \\
\hline & 118 & 6.07E-05 & $6.65 \mathrm{E}-05$ & $5.79 E-05$ & 5.99E-05 & $6.23 \mathrm{E}-05$ & 7.37E-05 & 8.61E-05 \\
\hline & 119 & $1.42 \mathrm{E}-03$ & $1.40 \mathrm{E}-03$ & $1.40 \mathrm{E}-03$ & $1.40 \mathrm{E}-03$ & $1.40 \mathrm{E}-03$ & $1.38 \mathrm{E}-03$ & $1.37 \mathrm{E}-03$ \\
\hline & 120 & $2.36 \mathrm{E}-04$ & $2.58 \mathrm{E}-04$ & $2.25 \mathrm{E}-04$ & $2.32 \mathrm{E}-04$ & $2.42 \mathrm{E}-04$ & $2.86 \mathrm{E}-04$ & $3.34 \mathrm{E}-04$ \\
\hline & 121 & 8.49E-05 & $9.31 \mathrm{E}-05$ & $8.16 \mathrm{E}-05$ & $8.45 \mathrm{E}-05$ & 8.80E-05 & $1.04 \mathrm{E}-04$ & $1.21 \mathrm{E}-04$ \\
\hline & 122 & 7.34E-04 & $7.26 \mathrm{E}-04$ & 7.19E-04 & $7.13 \mathrm{E}-04$ & $7.08 \mathrm{E}-04$ & 7.06E-04 & $7.04 \mathrm{E}-04$ \\
\hline & 123 & $3.86 \mathrm{E}-03$ & $3.74 \mathrm{E}-03$ & 3.79E-03 & $3.72 \mathrm{E}-03$ & 3.66E-03 & $3.49 \mathrm{E}-03$ & $3.31 \mathrm{E}-03$ \\
\hline & 124 & 9.37E-05 & $1.02 \mathrm{E}-04$ & 8.63E-05 & $8.86 \mathrm{E}-05$ & $9.18 \mathrm{E}-05$ & 1.11E-04 & $1.32 \mathrm{E}-04$ \\
\hline & 125 & $1.55 \mathrm{E}-04$ & $1.68 \mathrm{E}-04$ & $1.44 \mathrm{E}-04$ & $1.48 \mathrm{E}-04$ & $1.53 \mathrm{E}-04$ & $1.84 \mathrm{E}-04$ & $2.18 \mathrm{E}-04$ \\
\hline & 126 & $1.45 \mathrm{E}-04$ & $1.49 \mathrm{E}-04$ & $1.39 \mathrm{E}-04$ & $1.38 \mathrm{E}-04$ & $1.39 \mathrm{E}-04$ & $1.52 \mathrm{E}-04$ & $1.67 \mathrm{E}-04$ \\
\hline & 127 & $4.35 \mathrm{E}-04$ & $4.26 \mathrm{E}-04$ & 4.27E-04 & $4.23 \mathrm{E}-04$ & 4.19E-04 & 4.11E-04 & $4.02 \mathrm{E}-04$ \\
\hline & 128 & 8.69E-05 & $9.38 \mathrm{E}-05$ & 7.83E-05 & $8.02 \mathrm{E}-05$ & $8.28 \mathrm{E}-05$ & $1.01 \mathrm{E}-04$ & $1.21 \mathrm{E}-04$ \\
\hline & 129 & 8.61E-03 & $8.49 \mathrm{E}-03$ & $9.24 \mathrm{E}-03$ & 8.74E-03 & 8.16E-03 & 7.07E-03 & $5.83 \mathrm{E}-03$ \\
\hline & 130 & 4.83E-05 & $5.22 \mathrm{E}-05$ & 4.38E-05 & 4.49E-05 & 4.64E-05 & 5.65E-05 & $6.75 \mathrm{E}-05$ \\
\hline & 131 & $6.19 \mathrm{E}-04$ & $6.04 \mathrm{E}-04$ & $6.08 \mathrm{E}-04$ & $6.02 \mathrm{E}-04$ & 5.96E-04 & $5.79 \mathrm{E}-04$ & $5.61 \mathrm{E}-04$ \\
\hline & 132 & $3.13 E-05$ & 3.37E-05 & $2.78 \mathrm{E}-05$ & $2.83 \mathrm{E}-05$ & $2.92 \mathrm{E}-05$ & 3.61E-05 & 4.36E-05 \\
\hline & 133 & $8.43 \mathrm{E}-05$ & $8.41 \mathrm{E}-05$ & 8.03E-05 & $7.96 \mathrm{E}-05$ & 7.94E-05 & 8.31E-05 & $8.72 \mathrm{E}-05$ \\
\hline & 134 & $6.44 \mathrm{E}-04$ & 6.19E-04 & $6.29 \mathrm{E}-04$ & $6.13 \mathrm{E}-04$ & 5.97E-04 & $5.61 \mathrm{E}-04$ & $5.23 \mathrm{E}-04$ \\
\hline & 135 & $4.79 E-05$ & 5.16E-05 & $4.26 \mathrm{E}-05$ & 4.34E-05 & 4.47E-05 & 5.53E-05 & $6.68 \mathrm{E}-05$ \\
\hline & 136 & $2.60 \mathrm{E}-06$ & $2.78 \mathrm{E}-06$ & $2.26 \mathrm{E}-06$ & $2.29 \mathrm{E}-06$ & $2.35 \mathrm{E}-06$ & $2.95 \mathrm{E}-06$ & $3.60 \mathrm{E}-06$ \\
\hline & 137 & $5.90 \mathrm{E}-06$ & $5.95 \mathrm{E}-06$ & 5.56E-06 & 5.54E-06 & 5.55E-06 & 6.01E-06 & $6.50 \mathrm{E}-06$ \\
\hline & 138 & $1.48 \mathrm{E}-05$ & $1.44 \mathrm{E}-05$ & $1.42 \mathrm{E}-05$ & $1.39 \mathrm{E}-05$ & 1.37E-05 & $1.34 \mathrm{E}-05$ & $1.31 \mathrm{E}-05$ \\
\hline & 139 & $2.88 \mathrm{E}-06$ & $3.14 \mathrm{E}-06$ & $2.69 \mathrm{E}-06$ & $2.77 \mathrm{E}-06$ & $2.87 \mathrm{E}-06$ & $3.43 \mathrm{E}-06$ & 4.05E-06 \\
\hline
\end{tabular}


Table 5 (continued)

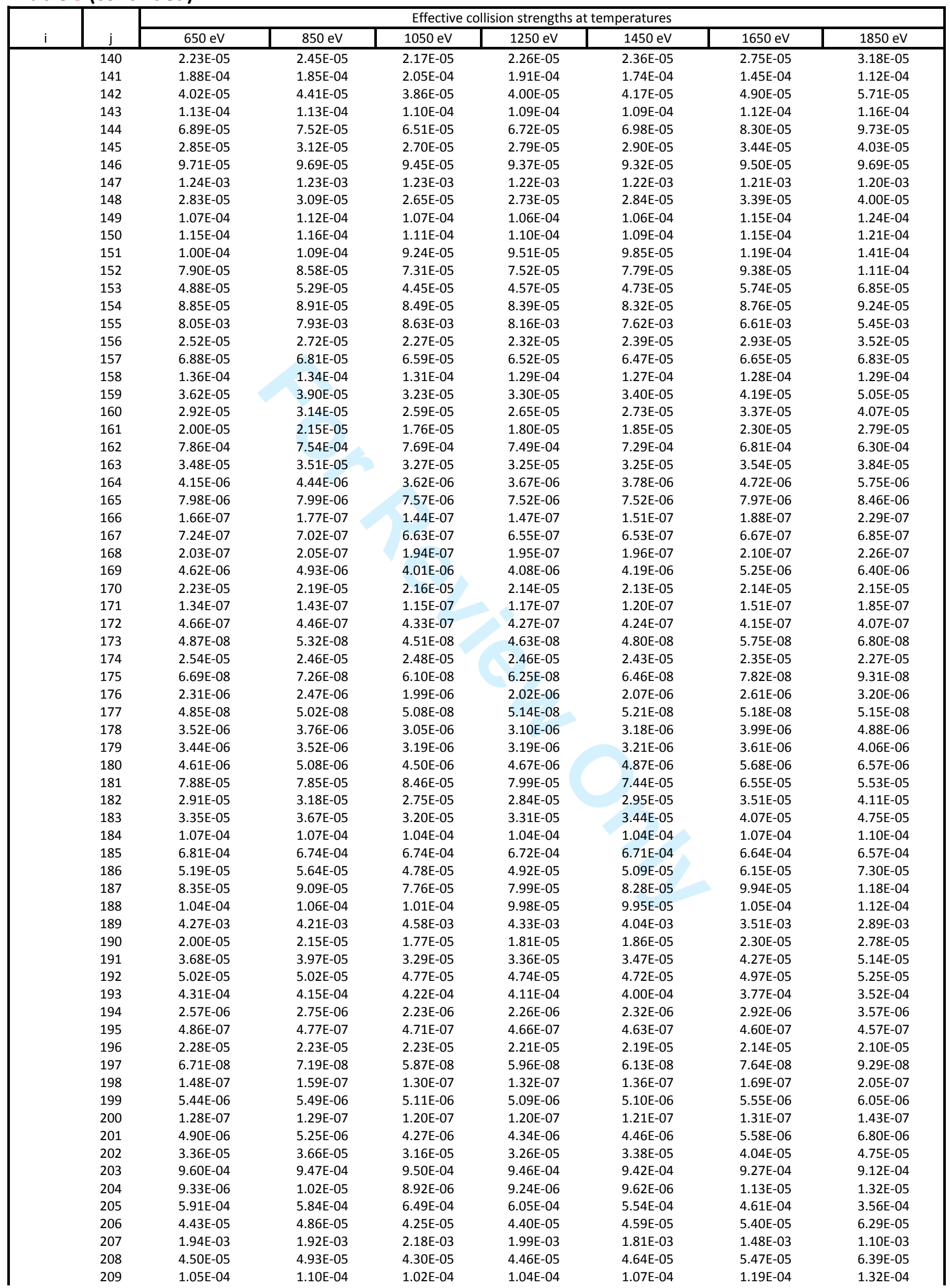


Table 5 (continued)

\begin{tabular}{|c|c|c|c|c|c|c|c|c|}
\hline \multirow[b]{2}{*}{$\mathrm{i}$} & \multirow[b]{2}{*}{ j } & \multicolumn{7}{|c|}{ Effective collision strengths at temperatures } \\
\hline & & $650 \mathrm{eV}$ & $850 \mathrm{eV}$ & $1050 \mathrm{eV}$ & $1250 \mathrm{eV}$ & $1450 \mathrm{eV}$ & $1650 \mathrm{eV}$ & $1850 \mathrm{eV}$ \\
\hline & 210 & $1.04 \mathrm{E}-04$ & $1.14 \mathrm{E}-04$ & 9.96E-05 & 1.03E-04 & $1.07 \mathrm{E}-04$ & $1.27 \mathrm{E}-04$ & $1.48 \mathrm{E}-04$ \\
\hline & 211 & $1.67 \mathrm{E}-03$ & 1.62E-03 & $1.64 \mathrm{E}-03$ & $1.61 \mathrm{E}-03$ & $1.58 \mathrm{E}-03$ & $1.51 \mathrm{E}-03$ & $1.43 \mathrm{E}-03$ \\
\hline & 212 & $3.06 \mathrm{E}-05$ & 3.31E-05 & $2.78 \mathrm{E}-05$ & $2.85 \mathrm{E}-05$ & $2.95 \mathrm{E}-05$ & 3.59E-05 & 4.29E-05 \\
\hline & 213 & 7.74E-05 & 7.93E-05 & 7.44E-05 & 7.49E-05 & 7.57E-05 & $8.23 \mathrm{E}-05$ & 8.96E-05 \\
\hline & 214 & $5.48 \mathrm{E}-05$ & $5.93 \mathrm{E}-05$ & $4.98 \mathrm{E}-05$ & $5.10 \mathrm{E}-05$ & $5.28 \mathrm{E}-05$ & $6.42 \mathrm{E}-05$ & 7.67E-05 \\
\hline & 215 & $4.82 \mathrm{E}-04$ & 4.69E-04 & 4.77E-04 & $4.71 \mathrm{E}-04$ & 4.66E-04 & 4.47E-04 & 4.26E-04 \\
\hline & 216 & $1.22 \mathrm{E}-05$ & 1.19E-05 & $1.14 \mathrm{E}-05$ & $1.13 \mathrm{E}-05$ & $1.12 \mathrm{E}-05$ & 1.16E-05 & 1.19E-05 \\
\hline & 217 & $3.24 \mathrm{E}-06$ & $3.46 \mathrm{E}-06$ & $2.81 \mathrm{E}-06$ & $2.85 \mathrm{E}-06$ & $2.93 \mathrm{E}-06$ & $3.68 \mathrm{E}-06$ & 4.49E-06 \\
\hline & 218 & $5.07 \mathrm{E}-06$ & $5.43 \mathrm{E}-06$ & 4.41E-06 & 4.47E-06 & 4.59E-06 & $5.76 \mathrm{E}-06$ & 7.03E-06 \\
\hline & 219 & $1.61 \mathrm{E}-05$ & $1.56 \mathrm{E}-05$ & $1.51 \mathrm{E}-05$ & $1.50 \mathrm{E}-05$ & $1.48 \mathrm{E}-05$ & $1.48 \mathrm{E}-05$ & 1.49E-05 \\
\hline & 220 & $1.96 \mathrm{E}-05$ & 2.14E-05 & $1.84 \mathrm{E}-05$ & $1.90 \mathrm{E}-05$ & $1.98 \mathrm{E}-05$ & $2.36 \mathrm{E}-05$ & 2.77E-05 \\
\hline & 221 & 4.74E-04 & 4.68E-04 & 4.70E-04 & $4.68 \mathrm{E}-04$ & 4.66E-04 & $4.58 \mathrm{E}-04$ & 4.50E-04 \\
\hline & 222 & $5.30 \mathrm{E}-06$ & $5.81 \mathrm{E}-06$ & $5.07 E-06$ & $5.25 \mathrm{E}-06$ & $5.47 \mathrm{E}-06$ & $6.44 \mathrm{E}-06$ & 7.51E-06 \\
\hline & 223 & 3.17E-04 & 3.14E-04 & $3.48 \mathrm{E}-04$ & $3.25 \mathrm{E}-04$ & $2.98 \mathrm{E}-04$ & $2.49 \mathrm{E}-04$ & $1.92 \mathrm{E}-04$ \\
\hline & 224 & $2.51 \mathrm{E}-05$ & $2.76 \mathrm{E}-05$ & $2.41 \mathrm{E}-05$ & $2.50 \mathrm{E}-05$ & $2.60 \mathrm{E}-05$ & $3.06 \mathrm{E}-05$ & 3.56E-05 \\
\hline & 225 & $1.03 \mathrm{E}-03$ & $1.02 \mathrm{E}-03$ & $1.16 \mathrm{E}-03$ & $1.06 \mathrm{E}-03$ & $9.63 \mathrm{E}-04$ & $7.86 \mathrm{E}-04$ & $5.85 \mathrm{E}-04$ \\
\hline & 226 & $2.39 \mathrm{E}-05$ & $2.61 \mathrm{E}-05$ & $2.28 \mathrm{E}-05$ & $2.36 \mathrm{E}-05$ & $2.46 \mathrm{E}-05$ & $2.90 \mathrm{E}-05$ & 3.39E-05 \\
\hline & 227 & 5.71E-05 & 5.97E-05 & $5.54 \mathrm{E}-05$ & $5.64 \mathrm{E}-05$ & 5.77E-05 & $6.40 \mathrm{E}-05$ & 7.10E-05 \\
\hline & 228 & $5.53 \mathrm{E}-05$ & $6.06 \mathrm{E}-05$ & $5.29 \mathrm{E}-05$ & 5.47E-05 & $5.70 \mathrm{E}-05$ & $6.72 \mathrm{E}-05$ & $7.84 \mathrm{E}-05$ \\
\hline & 229 & $8.10 \mathrm{E}-04$ & 7.83E-04 & $7.92 \mathrm{E}-04$ & 7.77E-04 & 7.64E-04 & $7.29 \mathrm{E}-04$ & $6.91 \mathrm{E}-04$ \\
\hline & 230 & $1.92 \mathrm{E}-05$ & $2.08 \mathrm{E}-05$ & $1.75 \mathrm{E}-05$ & $1.79 \mathrm{E}-05$ & $1.86 \mathrm{E}-05$ & $2.25 \mathrm{E}-05$ & $2.69 \mathrm{E}-05$ \\
\hline & 231 & 4.33E-05 & 4.47E-05 & 4.14E-05 & $4.18 \mathrm{E}-05$ & 4.24E-05 & $4.68 \mathrm{E}-05$ & 5.17E-05 \\
\hline & 232 & $3.44 \mathrm{E}-05$ & $3.72 \mathrm{E}-05$ & $3.13 \mathrm{E}-05$ & $3.21 \mathrm{E}-05$ & $3.32 \mathrm{E}-05$ & 4.04E-05 & $4.82 \mathrm{E}-05$ \\
\hline & 233 & $2.86 \mathrm{E}-04$ & $2.77 \mathrm{E}-04$ & $2.82 \mathrm{E}-04$ & $2.78 \mathrm{E}-04$ & $2.74 \mathrm{E}-04$ & $2.63 \mathrm{E}-04$ & $2.51 \mathrm{E}-04$ \\
\hline & 234 & $3.16 \mathrm{E}-06$ & $3.38 \mathrm{E}-06$ & $2.75 \mathrm{E}-06$ & $2.79 \mathrm{E}-06$ & $2.86 \mathrm{E}-06$ & $3.59 \mathrm{E}-06$ & $4.38 \mathrm{E}-06$ \\
\hline & 235 & $1.03 \mathrm{E}-05$ & $1.03 \mathrm{E}-05$ & $9.89 \mathrm{E}-06$ & $9.82 \mathrm{E}-06$ & $9.79 \mathrm{E}-06$ & $1.01 \mathrm{E}-05$ & $1.05 \mathrm{E}-05$ \\
\hline & 236 & 4.27E-07 & $4.55 \mathrm{E}-07$ & 4.11E-07 & $3.88 \mathrm{E}-07$ & $3.62 \mathrm{E}-07$ & $3.93 \mathrm{E}-07$ & 4.33E-07 \\
\hline & 237 & $1.57 \mathrm{E}-07$ & $1.64 \mathrm{E}-07$ & $1.31 \mathrm{E}-07$ & $1.30 \mathrm{E}-07$ & $1.32 \mathrm{E}-07$ & $1.72 \mathrm{E}-07$ & $2.18 \mathrm{E}-07$ \\
\hline & 238 & $4.95 \mathrm{E}-06$ & 5.30E-06 & 4.30E-06 & 4.37E-06 & 4.49E-06 & $5.62 \mathrm{E}-06$ & 6.87E-06 \\
\hline & 239 & $2.26 \mathrm{E}-07$ & $2.37 \mathrm{E}-07$ & $1.89 \mathrm{E}-07$ & $1.87 \mathrm{E}-07$ & $1.91 \mathrm{E}-07$ & $2.47 \mathrm{E}-07$ & $3.14 \mathrm{E}-07$ \\
\hline & 240 & $4.75 \mathrm{E}-07$ & 5.07E-07 & $4.61 \mathrm{E}-07$ & $4.34 \mathrm{E}-07$ & $4.01 \mathrm{E}-07$ & $4.28 \mathrm{E}-07$ & 4.66E-07 \\
\hline & 241 & $1.48 \mathrm{E}-05$ & $1.45 \mathrm{E}-05$ & $1.43 \mathrm{E}-05$ & $1.42 \mathrm{E}-05$ & $1.40 \mathrm{E}-05$ & $1.40 \mathrm{E}-05$ & $1.39 \mathrm{E}-05$ \\
\hline \multirow[t]{37}{*}{2} & 3 & $1.09 \mathrm{E}-02$ & $1.13 \mathrm{E}-02$ & $1.04 \mathrm{E}-02$ & $1.05 \mathrm{E}-02$ & 1.07E-02 & $1.20 \mathrm{E}-02$ & $1.33 \mathrm{E}-02$ \\
\hline & 4 & 5.89E-03 & 5.89E-03 & $5.75 \mathrm{E}-03$ & $5.74 \mathrm{E}-03$ & 5.75E-03 & $5.88 \mathrm{E}-03$ & $6.02 \mathrm{E}-03$ \\
\hline & 5 & $1.23 \mathrm{E}-02$ & $1.27 \mathrm{E}-02$ & $1.20 \mathrm{E}-02$ & $1.21 \mathrm{E}-02$ & $1.23 \mathrm{E}-02$ & $1.33 \mathrm{E}-02$ & $1.44 \mathrm{E}-02$ \\
\hline & 6 & $2.10 \mathrm{E}+00$ & $2.06 \mathrm{E}+00$ & $2.14 \mathrm{E}+00$ & $2.09 E+00$ & $2.03 E+00$ & $1.90 \mathrm{E}+00$ & $1.75 \mathrm{E}+00$ \\
\hline & 7 & $1.80 \mathrm{E}+00$ & $1.77 \mathrm{E}+00$ & $1.84 \mathrm{E}+00$ & $1.79 \mathrm{E}+00$ & $1.74 \mathrm{E}+00$ & $1.63 \mathrm{E}+00$ & $1.50 \mathrm{E}+00$ \\
\hline & 8 & $5.06 \mathrm{E}+00$ & $4.97 E+00$ & $5.17 \mathrm{E}+00$ & $5.04 \mathrm{E}+00$ & $4.90 E+00$ & $4.58 \mathrm{E}+00$ & $4.21 E+00$ \\
\hline & 9 & 4.94E-03 & $4.95 \mathrm{E}-03$ & $4.84 \mathrm{E}-03$ & $4.76 \mathrm{E}-03$ & 4.70E-03 & $4.81 \mathrm{E}-03$ & $4.92 \mathrm{E}-03$ \\
\hline & 10 & $1.92 \mathrm{E}+00$ & $1.88 \mathrm{E}+00$ & $1.96 \mathrm{E}+00$ & $1.91 \mathrm{E}+00$ & $1.85 \mathrm{E}+00$ & $1.73 \mathrm{E}+00$ & $1.59 \mathrm{E}+00$ \\
\hline & 11 & $3.24 \mathrm{E}-04$ & $3.44 \mathrm{E}-04$ & $2.75 \mathrm{E}-04$ & $2.78 \mathrm{E}-04$ & $2.85 \mathrm{E}-04$ & $3.63 \mathrm{E}-04$ & 4.47E-04 \\
\hline & 12 & $2.75 \mathrm{E}-03$ & $2.71 \mathrm{E}-03$ & $2.81 \mathrm{E}-03$ & $2.74 \mathrm{E}-03$ & $2.66 \mathrm{E}-03$ & $2.48 \mathrm{E}-03$ & $2.27 \mathrm{E}-03$ \\
\hline & 13 & 4.97E-02 & $4.88 \mathrm{E}-02$ & $5.08 \mathrm{E}-02$ & 4.94E-02 & 4.79E-02 & $4.46 \mathrm{E}-02$ & $4.08 \mathrm{E}-02$ \\
\hline & 14 & 7.01E-03 & $6.88 \mathrm{E}-03$ & 7.17E-03 & 6.97E-03 & $6.76 \mathrm{E}-03$ & $6.28 \mathrm{E}-03$ & $5.75 \mathrm{E}-03$ \\
\hline & 15 & $1.24 \mathrm{E}-04$ & $1.32 \mathrm{E}-04$ & $1.06 \mathrm{E}-04$ & $1.08 \mathrm{E}-04$ & $1.11 \mathrm{E}-04$ & $1.40 \mathrm{E}-04$ & $1.72 \mathrm{E}-04$ \\
\hline & 16 & $1.85 \mathrm{E}-02$ & $1.84 \mathrm{E}-02$ & $1.84 \mathrm{E}-02$ & $1.84 \mathrm{E}-02$ & $1.84 \mathrm{E}-02$ & $1.83 \mathrm{E}-02$ & $1.82 \mathrm{E}-02$ \\
\hline & 17 & 5.34E-02 & 5.31E-02 & $5.30 \mathrm{E}-02$ & $5.30 \mathrm{E}-02$ & $5.30 \mathrm{E}-02$ & $5.28 \mathrm{E}-02$ & $5.25 \mathrm{E}-02$ \\
\hline & 18 & $1.62 \mathrm{E}-01$ & $1.61 \mathrm{E}-01$ & $1.61 \mathrm{E}-01$ & $1.61 \mathrm{E}-01$ & $1.61 \mathrm{E}-01$ & $1.60 \mathrm{E}-01$ & 1.59E-01 \\
\hline & 19 & $7.60 \mathrm{E}-02$ & $7.55 \mathrm{E}-02$ & $7.54 \mathrm{E}-02$ & 7.54E-02 & $7.54 \mathrm{E}-02$ & $7.51 \mathrm{E}-02$ & $7.48 \mathrm{E}-02$ \\
\hline & 20 & 5.69E-02 & $5.66 \mathrm{E}-02$ & $5.63 \mathrm{E}-02$ & $5.63 \mathrm{E}-02$ & $5.64 \mathrm{E}-02$ & $5.65 \mathrm{E}-02$ & 5.66E-02 \\
\hline & 21 & $1.46 \mathrm{E}-02$ & $1.46 \mathrm{E}-02$ & $1.44 \mathrm{E}-02$ & $1.44 \mathrm{E}-02$ & $1.44 \mathrm{E}-02$ & $1.47 \mathrm{E}-02$ & $1.51 \mathrm{E}-02$ \\
\hline & 22 & 6.99E-02 & $6.95 \mathrm{E}-02$ & $6.92 \mathrm{E}-02$ & $6.92 \mathrm{E}-02$ & $6.93 \mathrm{E}-02$ & $6.92 \mathrm{E}-02$ & $6.92 \mathrm{E}-02$ \\
\hline & 23 & $2.31 \mathrm{E}-03$ & $2.36 \mathrm{E}-03$ & $2.18 \mathrm{E}-03$ & 2.19E-03 & $2.22 \mathrm{E}-03$ & $2.45 \mathrm{E}-03$ & $2.71 \mathrm{E}-03$ \\
\hline & 24 & $5.61 \mathrm{E}-05$ & $5.62 \mathrm{E}-05$ & $5.43 \mathrm{E}-05$ & $5.43 \mathrm{E}-05$ & $5.46 \mathrm{E}-05$ & $5.67 \mathrm{E}-05$ & $5.91 \mathrm{E}-05$ \\
\hline & 25 & $1.10 \mathrm{E}-03$ & $1.11 \mathrm{E}-03$ & $1.15 \mathrm{E}-03$ & $1.16 \mathrm{E}-03$ & $1.16 \mathrm{E}-03$ & $1.14 \mathrm{E}-03$ & $1.12 \mathrm{E}-03$ \\
\hline & 26 & $5.10 \mathrm{E}-04$ & $5.06 \mathrm{E}-04$ & $5.06 \mathrm{E}-04$ & $5.06 \mathrm{E}-04$ & $5.06 \mathrm{E}-04$ & $5.03 E-04$ & 5.00E-04 \\
\hline & 27 & $2.83 \mathrm{E}-04$ & $2.88 \mathrm{E}-04$ & $2.68 \mathrm{E}-04$ & $2.69 \mathrm{E}-04$ & $2.72 \mathrm{E}-04$ & 2.99E-04 & $3.28 \mathrm{E}-04$ \\
\hline & 28 & $5.26 \mathrm{E}-01$ & 5.17E-01 & $5.38 \mathrm{E}-01$ & $5.22 \mathrm{E}-01$ & $5.05 \mathrm{E}-01$ & 4.69E-01 & 4.29E-01 \\
\hline & 29 & $7.40 \mathrm{E}-04$ & $8.18 \mathrm{E}-04$ & $7.30 \mathrm{E}-04$ & $7.60 \mathrm{E}-04$ & 7.94E-04 & $9.20 \mathrm{E}-04$ & 1.06E-03 \\
\hline & 30 & $8.72 \mathrm{E}-06$ & $8.65 \mathrm{E}-06$ & $8.44 \mathrm{E}-06$ & $8.38 \mathrm{E}-06$ & 8.34E-06 & $8.48 \mathrm{E}-06$ & 8.63E-06 \\
\hline & 31 & $1.66 \mathrm{E}-05$ & $1.66 \mathrm{E}-05$ & $1.60 \mathrm{E}-05$ & $1.60 \mathrm{E}-05$ & $1.60 \mathrm{E}-05$ & $1.66 \mathrm{E}-05$ & $1.73 \mathrm{E}-05$ \\
\hline & 32 & $1.46 \mathrm{E}-04$ & $1.25 \mathrm{E}-04$ & $1.92 \mathrm{E}-04$ & $2.10 \mathrm{E}-04$ & $2.11 \mathrm{E}-04$ & $1.83 \mathrm{E}-04$ & $1.54 \mathrm{E}-04$ \\
\hline & 33 & $8.46 \mathrm{E}-06$ & 8.67E-06 & 8.07E-06 & $8.13 E-06$ & $8.23 \mathrm{E}-06$ & $9.04 \mathrm{E}-06$ & $9.92 \mathrm{E}-06$ \\
\hline & 34 & $8.02 \mathrm{E}-05$ & $7.93 \mathrm{E}-05$ & $7.92 \mathrm{E}-05$ & $7.86 \mathrm{E}-05$ & 7.81E-05 & $7.73 E-05$ & 7.64E-05 \\
\hline & 35 & $9.29 \mathrm{E}-05$ & $9.19 E-05$ & $9.16 \mathrm{E}-05$ & $9.09 E-05$ & $9.04 \mathrm{E}-05$ & 8.97E-05 & $8.90 \mathrm{E}-05$ \\
\hline & 36 & 7.97E-05 & 7.91E-05 & $7.82 \mathrm{E}-05$ & $7.78 \mathrm{E}-05$ & 7.74E-05 & $7.76 \mathrm{E}-05$ & $7.78 \mathrm{E}-05$ \\
\hline & 37 & $8.29 \mathrm{E}-06$ & $8.68 \mathrm{E}-06$ & 7.47E-06 & 7.51E-06 & 7.63E-06 & $9.27 \mathrm{E}-06$ & $1.11 \mathrm{E}-05$ \\
\hline & 38 & $1.04 \mathrm{E}-01$ & $1.11 \mathrm{E}-01$ & $1.55 \mathrm{E}-01$ & $1.62 \mathrm{E}-01$ & $1.63 \mathrm{E}-01$ & $1.52 \mathrm{E}-01$ & $1.35 \mathrm{E}-01$ \\
\hline & 39 & 4.19E-04 & $4.45 \mathrm{E}-04$ & $3.58 \mathrm{E}-04$ & $3.61 \mathrm{E}-04$ & $3.70 \mathrm{E}-04$ & 4.70E-04 & 5.79E-04 \\
\hline
\end{tabular}




\begin{tabular}{|c|c|c|c|c|c|c|c|c|}
\hline \multirow[b]{2}{*}{1} & \multirow[b]{2}{*}{$\mathrm{J}$} & \multicolumn{7}{|c|}{ Effective collision strengths at temperatures } \\
\hline & & $650 \mathrm{eV}$ & $850 \mathrm{eV}$ & $1050 \mathrm{eV}$ & $1250 \mathrm{eV}$ & $1450 \mathrm{eV}$ & $1650 \mathrm{eV}$ & $1850 \mathrm{eV}$ \\
\hline & 40 & $7.15 \mathrm{E}-02$ & 7.07E-02 & 7.94E-02 & $7.36 \mathrm{E}-02$ & $6.77 \mathrm{E}-02$ & $5.63 \mathrm{E}-02$ & $4.33 \mathrm{E}-02$ \\
\hline & 41 & $5.78 \mathrm{E}-02$ & $5.70 \mathrm{E}-02$ & $6.40 \mathrm{E}-02$ & $5.95 \mathrm{E}-02$ & $5.48 \mathrm{E}-02$ & $4.56 \mathrm{E}-02$ & $3.52 \mathrm{E}-02$ \\
\hline & 42 & $1.56 \mathrm{E}-01$ & $1.55 \mathrm{E}-01$ & $1.74 \mathrm{E}-01$ & $1.61 \mathrm{E}-01$ & $1.48 \mathrm{E}-01$ & $1.23 \mathrm{E}-01$ & $9.39 \mathrm{E}-02$ \\
\hline & 43 & 5.57E-04 & 5.69E-04 & $5.42 \mathrm{E}-04$ & $5.25 \mathrm{E}-04$ & 5.09E-04 & $5.44 \mathrm{E}-04$ & $5.80 \mathrm{E}-04$ \\
\hline & 44 & $5.38 \mathrm{E}-02$ & 5.31E-02 & $5.98 \mathrm{E}-02$ & $5.55 \mathrm{E}-02$ & $5.09 \mathrm{E}-02$ & 4.23E-02 & 3.25E-02 \\
\hline & 45 & $9.69 \mathrm{E}-05$ & $1.02 \mathrm{E}-04$ & 8.13E-05 & $8.08 \mathrm{E}-05$ & $8.25 E-05$ & $1.07 \mathrm{E}-04$ & $1.34 \mathrm{E}-04$ \\
\hline & 46 & $1.66 \mathrm{E}-05$ & $1.63 \mathrm{E}-05$ & $1.66 \mathrm{E}-05$ & $1.65 \mathrm{E}-05$ & $1.63 \mathrm{E}-05$ & $1.57 \mathrm{E}-05$ & $1.51 \mathrm{E}-05$ \\
\hline & 47 & 2.82E-05 & 2.78E-05 & $2.80 \mathrm{E}-05$ & 2.79E-05 & 2.77E-05 & $2.71 \mathrm{E}-05$ & $2.65 \mathrm{E}-05$ \\
\hline & 48 & $6.43 \mathrm{E}-03$ & $6.31 \mathrm{E}-03$ & $6.44 \mathrm{E}-03$ & $6.39 \mathrm{E}-03$ & $6.34 \mathrm{E}-03$ & $6.11 \mathrm{E}-03$ & 5.87E-03 \\
\hline & 49 & $1.88 \mathrm{E}-02$ & $1.84 \mathrm{E}-02$ & $1.88 \mathrm{E}-02$ & $1.87 \mathrm{E}-02$ & $1.85 \mathrm{E}-02$ & $1.79 \mathrm{E}-02$ & $1.71 \mathrm{E}-02$ \\
\hline & 50 & $5.57 \mathrm{E}-02$ & 5.47E-02 & $5.58 \mathrm{E}-02$ & $5.54 \mathrm{E}-02$ & $5.50 \mathrm{E}-02$ & 5.30E-02 & $5.08 \mathrm{E}-02$ \\
\hline & 51 & $1.94 \mathrm{E}-02$ & $1.90 \mathrm{E}-02$ & $1.94 \mathrm{E}-02$ & $1.92 \mathrm{E}-02$ & $1.91 \mathrm{E}-02$ & $1.85 \mathrm{E}-02$ & $1.78 \mathrm{E}-02$ \\
\hline & 52 & $2.83 \mathrm{E}-02$ & $2.78 \mathrm{E}-02$ & $2.84 \mathrm{E}-02$ & $2.82 \mathrm{E}-02$ & 2.79E-02 & $2.69 \mathrm{E}-02$ & $2.58 \mathrm{E}-02$ \\
\hline & 53 & $2.74 \mathrm{E}-03$ & $2.72 \mathrm{E}-03$ & 2.69E-03 & $2.68 \mathrm{E}-03$ & 2.67E-03 & $2.69 \mathrm{E}-03$ & $2.71 \mathrm{E}-03$ \\
\hline & 54 & $2.34 \mathrm{E}-02$ & $2.30 \mathrm{E}-02$ & $2.34 \mathrm{E}-02$ & $2.32 \mathrm{E}-02$ & $2.31 \mathrm{E}-02$ & $2.23 \mathrm{E}-02$ & $2.15 \mathrm{E}-02$ \\
\hline & 55 & $4.08 \mathrm{E}-04$ & 4.19E-04 & $3.74 \mathrm{E}-04$ & $3.74 \mathrm{E}-04$ & $3.78 \mathrm{E}-04$ & 4.37E-04 & $5.01 \mathrm{E}-04$ \\
\hline & 56 & $2.06 \mathrm{E}-05$ & 2.04E-05 & 2.09E-05 & $2.05 \mathrm{E}-05$ & $2.00 \mathrm{E}-05$ & $1.90 \mathrm{E}-05$ & $1.78 \mathrm{E}-05$ \\
\hline & 57 & $1.00 \mathrm{E}-04$ & $9.87 \mathrm{E}-05$ & $1.04 \mathrm{E}-04$ & $1.00 \mathrm{E}-04$ & $9.60 \mathrm{E}-05$ & 8.77E-05 & 7.82E-05 \\
\hline & 58 & 2.77E-04 & 2.74E-04 & $2.74 \mathrm{E}-04$ & 2.73E-04 & $2.72 \mathrm{E}-04$ & $2.68 \mathrm{E}-04$ & $2.64 \mathrm{E}-04$ \\
\hline & 59 & $1.18 \mathrm{E}-02$ & 1.17E-02 & $1.17 \mathrm{E}-02$ & 1.17E-02 & $1.16 \mathrm{E}-02$ & $1.15 \mathrm{E}-02$ & $1.13 \mathrm{E}-02$ \\
\hline & 60 & $9.92 \mathrm{E}-03$ & $9.89 \mathrm{E}-03$ & $9.66 \mathrm{E}-03$ & $9.64 \mathrm{E}-03$ & $9.64 \mathrm{E}-03$ & $9.84 \mathrm{E}-03$ & $1.01 \mathrm{E}-02$ \\
\hline & 61 & $1.57 \mathrm{E}-02$ & $1.55 \mathrm{E}-02$ & $1.55 \mathrm{E}-02$ & $1.55 \mathrm{E}-02$ & $1.54 \mathrm{E}-02$ & $1.53 \mathrm{E}-02$ & $1.51 \mathrm{E}-02$ \\
\hline & 62 & 4.35E-02 & 4.30E-02 & $4.30 \mathrm{E}-02$ & 4.29E-02 & 4.27E-02 & $4.22 \mathrm{E}-02$ & 4.16E-02 \\
\hline & 63 & 2.67E-02 & $2.64 \mathrm{E}-02$ & $2.64 \mathrm{E}-02$ & 2.63E-02 & $2.62 \mathrm{E}-02$ & $2.59 \mathrm{E}-02$ & $2.55 \mathrm{E}-02$ \\
\hline & 64 & 4.57E-03 & 4.56E-03 & $4.43 E-03$ & $4.42 \mathrm{E}-03$ & $4.42 \mathrm{E}-03$ & $4.55 \mathrm{E}-03$ & 4.70E-03 \\
\hline & 65 & $1.84 \mathrm{E}-03$ & $1.90 \mathrm{E}-03$ & $1.68 \mathrm{E}-03$ & $1.68 \mathrm{E}-03$ & $1.70 \mathrm{E}-03$ & $2.00 \mathrm{E}-03$ & $2.31 \mathrm{E}-03$ \\
\hline & 66 & 2.66E-02 & $2.64 \mathrm{E}-02$ & 2.63E-02 & 2.62E-02 & $2.62 \mathrm{E}-02$ & $2.59 \mathrm{E}-02$ & $2.57 \mathrm{E}-02$ \\
\hline & 67 & $2.56 \mathrm{E}-06$ & $2.70 \mathrm{E}-06$ & $2.15 \mathrm{E}-06$ & $2.16 \mathrm{E}-06$ & $2.20 \mathrm{E}-06$ & $2.83 \mathrm{E}-06$ & $3.51 \mathrm{E}-06$ \\
\hline & 68 & $1.02 \mathrm{E}-06$ & $1.04 \mathrm{E}-06$ & $1.02 \mathrm{E}-06$ & $9.96 \mathrm{E}-07$ & 9.64E-07 & $9.83 \mathrm{E}-07$ & $1.00 \mathrm{E}-06$ \\
\hline & 69 & $2.31 \mathrm{E}-05$ & $2.27 \mathrm{E}-05$ & $2.31 \mathrm{E}-05$ & $2.29 \mathrm{E}-05$ & $2.27 \mathrm{E}-05$ & $2.18 \mathrm{E}-05$ & $2.09 \mathrm{E}-05$ \\
\hline & 70 & 8.27E-06 & 8.13E-06 & $8.29 \mathrm{E}-06$ & $8.21 \mathrm{E}-06$ & $8.12 \mathrm{E}-06$ & $7.85 \mathrm{E}-06$ & $7.54 \mathrm{E}-06$ \\
\hline & 71 & $8.36 \mathrm{E}-06$ & $8.45 \mathrm{E}-06$ & $8.01 \mathrm{E}-06$ & $7.98 \mathrm{E}-06$ & $7.98 \mathrm{E}-06$ & $8.55 \mathrm{E}-06$ & $9.15 \mathrm{E}-06$ \\
\hline & 72 & $1.86 \mathrm{E}-06$ & $1.84 \mathrm{E}-06$ & $1.86 \mathrm{E}-06$ & $1.82 \mathrm{E}-06$ & $1.78 \mathrm{E}-06$ & $1.73 \mathrm{E}-06$ & $1.68 \mathrm{E}-06$ \\
\hline & 73 & $3.98 \mathrm{E}-06$ & $3.95 E-06$ & $3.95 \mathrm{E}-06$ & $3.94 \mathrm{E}-06$ & $3.93 E-06$ & $3.90 \mathrm{E}-06$ & $3.88 \mathrm{E}-06$ \\
\hline & 74 & $6.24 \mathrm{E}-06$ & $6.15 E-06$ & $6.47 \mathrm{E}-06$ & $6.24 \mathrm{E}-06$ & $5.98 \mathrm{E}-06$ & 5.47E-06 & 4.91E-06 \\
\hline & 75 & $5.16 \mathrm{E}-05$ & 5.06E-05 & 5.50E-05 & $5.20 \mathrm{E}-05$ & $4.85 \mathrm{E}-05$ & $4.18 \mathrm{E}-05$ & $3.43 \mathrm{E}-05$ \\
\hline & 76 & $2.91 \mathrm{E}-02$ & $2.84 \mathrm{E}-02$ & $2.76 \mathrm{E}-02$ & $2.75 \mathrm{E}-02$ & $2.75 \mathrm{E}-02$ & $2.74 \mathrm{E}-02$ & $2.74 \mathrm{E}-02$ \\
\hline & 77 & $1.41 \mathrm{E}-04$ & $1.51 \mathrm{E}-04$ & $1.22 \mathrm{E}-04$ & $1.24 \mathrm{E}-04$ & $1.27 \mathrm{E}-04$ & $1.60 \mathrm{E}-04$ & $1.96 \mathrm{E}-04$ \\
\hline & 78 & $1.07 \mathrm{E}-03$ & $1.05 \mathrm{E}-03$ & $1.16 \mathrm{E}-03$ & $1.09 \mathrm{E}-03$ & $1.01 \mathrm{E}-03$ & $8.48 \mathrm{E}-04$ & 6.67E-04 \\
\hline & 79 & 5.79E-07 & $6.21 \mathrm{E}-07$ & 5.14E-07 & $5.24 \mathrm{E}-07$ & $5.40 \mathrm{E}-07$ & $6.68 \mathrm{E}-07$ & 8.07E-07 \\
\hline & 80 & 1.19E-02 & 1.17E-02 & $1.29 \mathrm{E}-02$ & $1.21 \mathrm{E}-02$ & $1.12 \mathrm{E}-02$ & $9.49 \mathrm{E}-03$ & 7.51E-03 \\
\hline & 81 & $1.31 \mathrm{E}-02$ & $1.28 \mathrm{E}-02$ & $1.41 \mathrm{E}-02$ & $1.33 \mathrm{E}-02$ & $1.23 \mathrm{E}-02$ & $1.03 \mathrm{E}-02$ & $8.12 \mathrm{E}-03$ \\
\hline & 82 & $3.32 \mathrm{E}-02$ & $3.26 \mathrm{E}-02$ & 3.60E-02 & $3.38 \mathrm{E}-02$ & $3.12 \mathrm{E}-02$ & $2.62 \mathrm{E}-02$ & $2.05 \mathrm{E}-02$ \\
\hline & 83 & $9.55 \mathrm{E}-04$ & $9.45 \mathrm{E}-04$ & $1.01 \mathrm{E}-03$ & $9.56 \mathrm{E}-04$ & 8.91E-04 & $7.93 \mathrm{E}-04$ & $6.79 \mathrm{E}-04$ \\
\hline & 84 & $1.18 \mathrm{E}-02$ & 1.16E-02 & $1.28 \mathrm{E}-02$ & $1.20 \mathrm{E}-02$ & $1.11 \mathrm{E}-02$ & $9.38 \mathrm{E}-03$ & $7.40 \mathrm{E}-03$ \\
\hline & 85 & 4.40E-05 & 4.63E-05 & 3.70E-05 & 3.67E-05 & $3.75 \mathrm{E}-05$ & $4.84 \mathrm{E}-05$ & $6.10 \mathrm{E}-05$ \\
\hline & 86 & $3.55 E-04$ & $3.43 E-04$ & $3.42 \mathrm{E}-04$ & 3.39E-04 & $3.37 \mathrm{E}-04$ & $3.29 \mathrm{E}-04$ & $3.21 \mathrm{E}-04$ \\
\hline & 87 & $9.58 \mathrm{E}-04$ & $9.27 \mathrm{E}-04$ & $9.22 \mathrm{E}-04$ & $9.14 \mathrm{E}-04$ & $9.10 \mathrm{E}-04$ & $8.88 \mathrm{E}-04$ & $8.65 \mathrm{E}-04$ \\
\hline & 88 & $1.24 \mathrm{E}-03$ & $1.20 \mathrm{E}-03$ & $1.20 \mathrm{E}-03$ & $1.19 \mathrm{E}-03$ & $1.18 \mathrm{E}-03$ & $1.15 \mathrm{E}-03$ & $1.12 \mathrm{E}-03$ \\
\hline & 89 & $3.63 \mathrm{E}-03$ & $3.52 \mathrm{E}-03$ & 3.50E-03 & $3.47 \mathrm{E}-03$ & $3.45 \mathrm{E}-03$ & $3.37 \mathrm{E}-03$ & $3.29 \mathrm{E}-03$ \\
\hline & 90 & $6.06 \mathrm{E}-03$ & 5.87E-03 & $5.83 \mathrm{E}-03$ & 5.79E-03 & $5.76 \mathrm{E}-03$ & $5.63 \mathrm{E}-03$ & $5.49 \mathrm{E}-03$ \\
\hline & 91 & 4.93E-03 & 4.78E-03 & 4.73E-03 & 4.70E-03 & $4.68 \mathrm{E}-03$ & $4.61 \mathrm{E}-03$ & $4.53 \mathrm{E}-03$ \\
\hline & 92 & $1.41 \mathrm{E}-02$ & 1.36E-02 & $1.36 \mathrm{E}-02$ & $1.34 \mathrm{E}-02$ & $1.34 \mathrm{E}-02$ & $1.31 \mathrm{E}-02$ & $1.27 \mathrm{E}-02$ \\
\hline & 93 & $1.28 \mathrm{E}-03$ & $1.25 \mathrm{E}-03$ & $1.22 \mathrm{E}-03$ & $1.21 \mathrm{E}-03$ & $1.21 \mathrm{E}-03$ & $1.22 \mathrm{E}-03$ & $1.24 \mathrm{E}-03$ \\
\hline & 94 & $6.14 \mathrm{E}-03$ & $5.95 \mathrm{E}-03$ & $5.90 \mathrm{E}-03$ & $5.86 \mathrm{E}-03$ & $5.83 \mathrm{E}-03$ & $5.72 \mathrm{E}-03$ & $5.61 \mathrm{E}-03$ \\
\hline & 95 & $6.88 \mathrm{E}-04$ & $6.68 \mathrm{E}-04$ & $6.63 \mathrm{E}-04$ & $6.58 \mathrm{E}-04$ & $6.56 \mathrm{E}-04$ & $6.45 \mathrm{E}-04$ & $6.33 \mathrm{E}-04$ \\
\hline & 96 & $1.85 \mathrm{E}-04$ & $1.82 \mathrm{E}-04$ & $1.76 \mathrm{E}-04$ & $1.75 \mathrm{E}-04$ & $1.75 \mathrm{E}-04$ & $1.78 \mathrm{E}-04$ & $1.83 \mathrm{E}-04$ \\
\hline & 97 & 8.57E-05 & 9.09E-05 & $7.32 \mathrm{E}-05$ & 7.39E-05 & 7.57E-05 & $9.62 \mathrm{E}-05$ & 1.19E-04 \\
\hline & 98 & $1.78 \mathrm{E}-03$ & $1.75 \mathrm{E}-03$ & $1.75 \mathrm{E}-03$ & $1.74 \mathrm{E}-03$ & $1.73 \mathrm{E}-03$ & $1.72 \mathrm{E}-03$ & $1.70 \mathrm{E}-03$ \\
\hline & 99 & 2.57E-03 & 2.54E-03 & $2.53 \mathrm{E}-03$ & $2.51 \mathrm{E}-03$ & $2.50 \mathrm{E}-03$ & $2.49 \mathrm{E}-03$ & 2.47E-03 \\
\hline & 100 & $1.80 \mathrm{E}-03$ & 1.80E-03 & $1.72 \mathrm{E}-03$ & $1.72 \mathrm{E}-03$ & $1.72 \mathrm{E}-03$ & $1.80 \mathrm{E}-03$ & $1.89 \mathrm{E}-03$ \\
\hline & 101 & $6.60 \mathrm{E}-03$ & $6.50 \mathrm{E}-03$ & $6.49 \mathrm{E}-03$ & $6.45 \mathrm{E}-03$ & $6.42 \mathrm{E}-03$ & $6.36 \mathrm{E}-03$ & $6.29 \mathrm{E}-03$ \\
\hline & 102 & 3.61E-03 & 3.56E-03 & $3.54 \mathrm{E}-03$ & $3.52 \mathrm{E}-03$ & $3.50 \mathrm{E}-03$ & $3.48 \mathrm{E}-03$ & $3.46 \mathrm{E}-03$ \\
\hline & 103 & $2.02 \mathrm{E}-03$ & 1.99E-03 & $2.00 \mathrm{E}-03$ & $1.99 \mathrm{E}-03$ & $1.99 \mathrm{E}-03$ & $1.96 \mathrm{E}-03$ & $1.93 \mathrm{E}-03$ \\
\hline & 104 & $1.40 \mathrm{E}-03$ & 1.39E-03 & $1.37 \mathrm{E}-03$ & $1.37 \mathrm{E}-03$ & $1.37 \mathrm{E}-03$ & $1.39 \mathrm{E}-03$ & $1.40 \mathrm{E}-03$ \\
\hline & 105 & $9.71 \mathrm{E}-04$ & $9.81 \mathrm{E}-04$ & $9.17 E-04$ & $9.17 \mathrm{E}-04$ & $9.21 \mathrm{E}-04$ & 9.99E-04 & $1.08 \mathrm{E}-03$ \\
\hline & 106 & $6.88 \mathrm{E}-04$ & $6.95 \mathrm{E}-04$ & $6.49 \mathrm{E}-04$ & $6.49 \mathrm{E}-04$ & $6.52 \mathrm{E}-04$ & 7.09E-04 & 7.71E-04 \\
\hline & 107 & 4.09E-03 & 4.03E-03 & 4.00E-03 & $3.98 \mathrm{E}-03$ & $3.96 \mathrm{E}-03$ & $3.96 \mathrm{E}-03$ & $3.96 \mathrm{E}-03$ \\
\hline & 108 & $1.27 \mathrm{E}-03$ & 1.27E-03 & $1.22 \mathrm{E}-03$ & $1.22 \mathrm{E}-03$ & $1.22 \mathrm{E}-03$ & $1.28 \mathrm{E}-03$ & $1.35 \mathrm{E}-03$ \\
\hline & 109 & $1.63 \mathrm{E}-03$ & $1.61 \mathrm{E}-03$ & $1.60 \mathrm{E}-03$ & $1.59 \mathrm{E}-03$ & $1.59 \mathrm{E}-03$ & $1.60 \mathrm{E}-03$ & $1.61 \mathrm{E}-03$ \\
\hline
\end{tabular}


Table 5 (continued)

\begin{tabular}{|c|c|c|c|c|c|c|c|c|}
\hline \multirow[b]{2}{*}{$\mathrm{i}$} & \multirow[b]{2}{*}{$j$} & \multicolumn{7}{|c|}{ Effective collision strengths at temperatures } \\
\hline & & $650 \mathrm{eV}$ & $850 \mathrm{eV}$ & $1050 \mathrm{eV}$ & $1250 \mathrm{eV}$ & $1450 \mathrm{eV}$ & $1650 \mathrm{eV}$ & $1850 \mathrm{eV}$ \\
\hline & 110 & $5.18 \mathrm{E}-03$ & $5.10 \mathrm{E}-03$ & $5.13 \mathrm{E}-03$ & $5.11 \mathrm{E}-03$ & $5.10 \mathrm{E}-03$ & $5.03 \mathrm{E}-03$ & $4.95 \mathrm{E}-03$ \\
\hline & 111 & $2.76 \mathrm{E}-03$ & $2.72 \mathrm{E}-03$ & $2.72 \mathrm{E}-03$ & $2.71 \mathrm{E}-03$ & $2.71 \mathrm{E}-03$ & $2.69 \mathrm{E}-03$ & 2.67E-03 \\
\hline & 112 & $1.09 \mathrm{E}-03$ & 1.09E-03 & $1.05 \mathrm{E}-03$ & $1.05 \mathrm{E}-03$ & $1.05 \mathrm{E}-03$ & $1.10 \mathrm{E}-03$ & $1.15 \mathrm{E}-03$ \\
\hline & 113 & $3.42 \mathrm{E}-03$ & $3.38 \mathrm{E}-03$ & $3.38 \mathrm{E}-03$ & $3.37 \mathrm{E}-03$ & 3.37E-03 & $3.34 \mathrm{E}-03$ & $3.31 \mathrm{E}-03$ \\
\hline & 114 & 7.74E-08 & $7.45 \mathrm{E}-08$ & $7.78 \mathrm{E}-08$ & 7.79E-08 & $7.82 \mathrm{E}-08$ & $7.48 \mathrm{E}-08$ & 7.06E-08 \\
\hline & 115 & 4.07E-07 & 4.14E-07 & 3.82E-07 & $3.84 \mathrm{E}-07$ & 3.87E-07 & 4.32E-07 & 4.79E-07 \\
\hline & 116 & $6.92 \mathrm{E}-06$ & $6.73 \mathrm{E}-06$ & $6.59 \mathrm{E}-06$ & $6.53 \mathrm{E}-06$ & $6.49 \mathrm{E}-06$ & $6.43 \mathrm{E}-06$ & 6.37E-06 \\
\hline & 117 & $1.24 \mathrm{E}-05$ & $1.20 \mathrm{E}-05$ & 1.19E-05 & 1.17E-05 & $1.15 \mathrm{E}-05$ & $1.12 \mathrm{E}-05$ & 1.10E-05 \\
\hline & 118 & $1.55 \mathrm{E}-06$ & 1.49E-06 & $1.45 \mathrm{E}-06$ & $1.46 \mathrm{E}-06$ & $1.48 \mathrm{E}-06$ & $1.49 \mathrm{E}-06$ & $1.49 \mathrm{E}-06$ \\
\hline & 119 & $3.43 \mathrm{E}-07$ & $3.62 \mathrm{E}-07$ & $2.88 \mathrm{E}-07$ & $2.89 \mathrm{E}-07$ & $2.95 \mathrm{E}-07$ & 3.79E-07 & $4.72 \mathrm{E}-07$ \\
\hline & 120 & 3.14E-05 & 3.07E-05 & 3.16E-05 & 3.03E-05 & 2.90E-05 & 2.69E-05 & $2.45 \mathrm{E}-05$ \\
\hline & 121 & $1.74 \mathrm{E}-05$ & $1.67 \mathrm{E}-05$ & $1.56 \mathrm{E}-05$ & $1.53 \mathrm{E}-05$ & $1.51 \mathrm{E}-05$ & $1.52 \mathrm{E}-05$ & $1.54 \mathrm{E}-05$ \\
\hline & 122 & $1.93 \mathrm{E}-05$ & $1.85 \mathrm{E}-05$ & $1.75 \mathrm{E}-05$ & $1.71 \mathrm{E}-05$ & 1.69E-05 & $1.68 \mathrm{E}-05$ & $1.68 \mathrm{E}-05$ \\
\hline & 123 & 1.49E-06 & $1.53 \mathrm{E}-06$ & $1.33 \mathrm{E}-06$ & $1.31 \mathrm{E}-06$ & $1.30 \mathrm{E}-06$ & $1.52 \mathrm{E}-06$ & 1.76E-06 \\
\hline & 124 & $3.13 \mathrm{E}-07$ & $3.29 E-07$ & $3.11 \mathrm{E}-07$ & 2.91E-07 & $2.66 \mathrm{E}-07$ & 2.69E-07 & 2.79E-07 \\
\hline & 125 & $4.92 \mathrm{E}-06$ & $5.25 \mathrm{E}-06$ & 4.91E-06 & 4.59E-06 & 4.19E-06 & $4.15 E-06$ & $4.25 \mathrm{E}-06$ \\
\hline & 126 & 6.04E-07 & $5.83 \mathrm{E}-07$ & 5.55E-07 & 5.54E-07 & $5.58 \mathrm{E}-07$ & $5.74 \mathrm{E}-07$ & $5.93 \mathrm{E}-07$ \\
\hline & 127 & $1.68 \mathrm{E}-05$ & $1.64 \mathrm{E}-05$ & $1.62 \mathrm{E}-05$ & $1.59 \mathrm{E}-05$ & $1.56 \mathrm{E}-05$ & $1.54 \mathrm{E}-05$ & $1.52 \mathrm{E}-05$ \\
\hline & 128 & $9.49 \mathrm{E}-05$ & $9.26 \mathrm{E}-05$ & 9.37E-05 & $9.27 \mathrm{E}-05$ & $9.16 \mathrm{E}-05$ & 8.83E-05 & $8.48 \mathrm{E}-05$ \\
\hline & 129 & $6.52 \mathrm{E}-07$ & $6.62 \mathrm{E}-07$ & $5.79 \mathrm{E}-07$ & $5.82 \mathrm{E}-07$ & $5.94 \mathrm{E}-07$ & $6.94 \mathrm{E}-07$ & $8.03 E-07$ \\
\hline & 130 & $6.13 \mathrm{E}-06$ & $5.81 \mathrm{E}-06$ & $5.27 \mathrm{E}-06$ & 4.99E-06 & 4.74E-06 & $4.74 \mathrm{E}-06$ & $4.80 \mathrm{E}-06$ \\
\hline & 131 & 1.14E-05 & $1.11 \mathrm{E}-05$ & $1.09 \mathrm{E}-05$ & $1.08 \mathrm{E}-05$ & $1.06 \mathrm{E}-05$ & $1.05 \mathrm{E}-05$ & $1.04 \mathrm{E}-05$ \\
\hline & 132 & 2.09E-07 & $2.02 \mathrm{E}-07$ & $1.98 \mathrm{E}-07$ & $1.92 \mathrm{E}-07$ & $1.86 \mathrm{E}-07$ & $1.83 \mathrm{E}-07$ & $1.80 \mathrm{E}-07$ \\
\hline & 133 & $2.72 \mathrm{E}-07$ & $2.46 \mathrm{E}-07$ & $1.98 \mathrm{E}-07$ & $1.93 \mathrm{E}-07$ & $1.94 \mathrm{E}-07$ & $2.15 \mathrm{E}-07$ & $2.42 \mathrm{E}-07$ \\
\hline & 134 & $1.05 \mathrm{E}-06$ & $1.02 \mathrm{E}-06$ & $1.09 \mathrm{E}-06$ & $1.03 E-06$ & $9.50 \mathrm{E}-07$ & $8.23 E-07$ & $6.78 \mathrm{E}-07$ \\
\hline & 135 & 1.19E-05 & 1.17E-05 & 1.29E-05 & $1.21 \mathrm{E}-05$ & $1.11 \mathrm{E}-05$ & $9.24 \mathrm{E}-06$ & 7.13E-06 \\
\hline & 136 & $5.44 \mathrm{E}-08$ & $6.07 \mathrm{E}-08$ & $6.22 \mathrm{E}-08$ & $5.96 \mathrm{E}-08$ & $4.83 \mathrm{E}-08$ & 4.53E-08 & $4.91 \mathrm{E}-08$ \\
\hline & 137 & 4.73E-08 & $4.51 \mathrm{E}-08$ & 3.94E-08 & 4.04E-08 & $4.23 \mathrm{E}-08$ & $4.91 \mathrm{E}-08$ & $5.62 \mathrm{E}-08$ \\
\hline & 138 & $6.75 \mathrm{E}-07$ & $6.54 \mathrm{E}-07$ & $6.27 \mathrm{E}-07$ & $6.24 \mathrm{E}-07$ & $6.25 \mathrm{E}-07$ & $6.40 \mathrm{E}-07$ & $6.57 \mathrm{E}-07$ \\
\hline & 139 & 5.33E-06 & $5.18 \mathrm{E}-06$ & $5.22 \mathrm{E}-06$ & $5.18 \mathrm{E}-06$ & $5.15 \mathrm{E}-06$ & $5.01 \mathrm{E}-06$ & $4.86 \mathrm{E}-06$ \\
\hline & 140 & $1.01 \mathrm{E}-02$ & $9.96 \mathrm{E}-03$ & $9.96 \mathrm{E}-03$ & $9.99 \mathrm{E}-03$ & $1.00 \mathrm{E}-02$ & $9.95 \mathrm{E}-03$ & $9.88 \mathrm{E}-03$ \\
\hline & 141 & 7.31E-05 & 7.81E-05 & $6.32 \mathrm{E}-05$ & $6.40 \mathrm{E}-05$ & $6.56 \mathrm{E}-05$ & 8.27E-05 & $1.01 \mathrm{E}-04$ \\
\hline & 142 & $5.62 \mathrm{E}-03$ & $5.48 \mathrm{E}-03$ & 5.99E-03 & $5.62 \mathrm{E}-03$ & $5.21 \mathrm{E}-03$ & $4.42 \mathrm{E}-03$ & $3.53 \mathrm{E}-03$ \\
\hline & 143 & 4.67E-03 & $4.56 \mathrm{E}-03$ & 4.96E-03 & $4.66 \mathrm{E}-03$ & 4.33E-03 & 3.70E-03 & $2.99 \mathrm{E}-03$ \\
\hline & 144 & 1.27E-02 & $1.24 \mathrm{E}-02$ & $1.35 \mathrm{E}-02$ & $1.27 \mathrm{E}-02$ & $1.18 \mathrm{E}-02$ & $9.99 \mathrm{E}-03$ & 7.97E-03 \\
\hline & 145 & $1.45 \mathrm{E}-04$ & $1.47 \mathrm{E}-04$ & $1.43 \mathrm{E}-04$ & $1.38 \mathrm{E}-04$ & $1.32 \mathrm{E}-04$ & $1.35 \mathrm{E}-04$ & $1.38 \mathrm{E}-04$ \\
\hline & 146 & $4.54 \mathrm{E}-03$ & $4.43 \mathrm{E}-03$ & $4.82 \mathrm{E}-03$ & $4.53 \mathrm{E}-03$ & $4.21 \mathrm{E}-03$ & $3.60 \mathrm{E}-03$ & $2.91 \mathrm{E}-03$ \\
\hline & 147 & $2.46 \mathrm{E}-05$ & $2.59 \mathrm{E}-05$ & 2.07E-05 & $2.05 \mathrm{E}-05$ & $2.10 \mathrm{E}-05$ & $2.71 \mathrm{E}-05$ & $3.40 \mathrm{E}-05$ \\
\hline & 148 & $6.25 \mathrm{E}-04$ & $6.07 \mathrm{E}-04$ & 6.03E-04 & $5.98 \mathrm{E}-04$ & 5.95E-04 & $5.82 \mathrm{E}-04$ & $5.68 \mathrm{E}-04$ \\
\hline & 149 & $1.86 \mathrm{E}-03$ & $1.81 \mathrm{E}-03$ & $1.80 \mathrm{E}-03$ & $1.78 \mathrm{E}-03$ & $1.77 \mathrm{E}-03$ & $1.73 \mathrm{E}-03$ & $1.69 \mathrm{E}-03$ \\
\hline & 150 & 2.00E-03 & $1.95 \mathrm{E}-03$ & $1.92 \mathrm{E}-03$ & $1.91 \mathrm{E}-03$ & $1.90 \mathrm{E}-03$ & $1.88 \mathrm{E}-03$ & $1.86 \mathrm{E}-03$ \\
\hline & 151 & 5.57E-03 & $5.41 \mathrm{E}-03$ & $5.38 \mathrm{E}-03$ & $5.33 \mathrm{E}-03$ & 5.30E-03 & $5.18 \mathrm{E}-03$ & $5.06 \mathrm{E}-03$ \\
\hline & 152 & 3.07E-03 & $2.98 \mathrm{E}-03$ & $2.96 \mathrm{E}-03$ & $2.94 \mathrm{E}-03$ & 2.92E-03 & $2.86 \mathrm{E}-03$ & $2.79 \mathrm{E}-03$ \\
\hline & 153 & $1.45 \mathrm{E}-04$ & $1.49 \mathrm{E}-04$ & $1.31 \mathrm{E}-04$ & $1.32 \mathrm{E}-04$ & $1.33 \mathrm{E}-04$ & $1.57 \mathrm{E}-04$ & $1.82 \mathrm{E}-04$ \\
\hline & 154 & $2.49 \mathrm{E}-03$ & $2.42 \mathrm{E}-03$ & $2.40 \mathrm{E}-03$ & $2.38 \mathrm{E}-03$ & $2.37 \mathrm{E}-03$ & $2.33 \mathrm{E}-03$ & $2.30 \mathrm{E}-03$ \\
\hline & 155 & 7.26E-05 & 7.59E-05 & $6.42 \mathrm{E}-05$ & 6.47E-05 & $6.60 \mathrm{E}-05$ & $8.16 \mathrm{E}-05$ & $9.86 \mathrm{E}-05$ \\
\hline & 156 & 6.07E-04 & 5.99E-04 & 5.97E-04 & $5.93 \mathrm{E}-04$ & $5.90 \mathrm{E}-04$ & $5.85 \mathrm{E}-04$ & $5.80 \mathrm{E}-04$ \\
\hline & 157 & $6.74 \mathrm{E}-04$ & $6.78 \mathrm{E}-04$ & $6.43 \mathrm{E}-04$ & $6.41 \mathrm{E}-04$ & $6.41 \mathrm{E}-04$ & $6.82 \mathrm{E}-04$ & $7.26 \mathrm{E}-04$ \\
\hline & 158 & $9.16 \mathrm{E}-04$ & $9.05 \mathrm{E}-04$ & 8.97E-04 & 8.91E-04 & 8.86E-04 & 8.83E-04 & $8.80 \mathrm{E}-04$ \\
\hline & 159 & $2.26 \mathrm{E}-03$ & $2.22 \mathrm{E}-03$ & 2.19E-03 & 2.17E-03 & $2.16 \mathrm{E}-03$ & $2.15 \mathrm{E}-03$ & $2.13 \mathrm{E}-03$ \\
\hline & 160 & $1.41 \mathrm{E}-03$ & $1.39 \mathrm{E}-03$ & 1.39E-03 & $1.37 \mathrm{E}-03$ & $1.37 \mathrm{E}-03$ & $1.35 \mathrm{E}-03$ & $1.34 \mathrm{E}-03$ \\
\hline & 161 & $2.12 \mathrm{E}-04$ & $2.22 \mathrm{E}-04$ & 1.87E-04 & $1.89 \mathrm{E}-04$ & $1.92 \mathrm{E}-04$ & $2.38 \mathrm{E}-04$ & $2.88 \mathrm{E}-04$ \\
\hline & 162 & $2.39 \mathrm{E}-04$ & $2.45 \mathrm{E}-04$ & $2.23 \mathrm{E}-04$ & $2.23 \mathrm{E}-04$ & $2.25 \mathrm{E}-04$ & $2.54 \mathrm{E}-04$ & $2.86 \mathrm{E}-04$ \\
\hline & 163 & $1.40 \mathrm{E}-03$ & $1.39 \mathrm{E}-03$ & 1.37E-03 & $1.36 \mathrm{E}-03$ & $1.35 \mathrm{E}-03$ & $1.36 \mathrm{E}-03$ & $1.37 \mathrm{E}-03$ \\
\hline & 164 & $1.04 \mathrm{E}-03$ & $1.02 \mathrm{E}-03$ & $1.01 \mathrm{E}-03$ & $1.01 \mathrm{E}-03$ & $1.00 \mathrm{E}-03$ & $9.92 \mathrm{E}-04$ & $9.81 \mathrm{E}-04$ \\
\hline & 165 & 7.12E-04 & 7.11E-04 & 6.69E-04 & 6.67E-04 & $6.68 \mathrm{E}-04$ & $7.13 E-04$ & $7.63 \mathrm{E}-04$ \\
\hline & 166 & $1.81 \mathrm{E}-04$ & $1.82 \mathrm{E}-04$ & $1.81 \mathrm{E}-04$ & $1.80 \mathrm{E}-04$ & $1.78 \mathrm{E}-04$ & $1.78 \mathrm{E}-04$ & $1.78 \mathrm{E}-04$ \\
\hline & 167 & $1.21 \mathrm{E}-04$ & $1.22 \mathrm{E}-04$ & 1.17E-04 & 1.17E-04 & $1.16 \mathrm{E}-04$ & $1.21 \mathrm{E}-04$ & $1.27 \mathrm{E}-04$ \\
\hline & 168 & 1.16E-04 & 1.17E-04 & 1.09E-04 & $1.08 \mathrm{E}-04$ & $1.08 \mathrm{E}-04$ & 1.19E-04 & $1.30 \mathrm{E}-04$ \\
\hline & 169 & 2.71E-03 & $2.65 \mathrm{E}-03$ & 2.63E-03 & $2.61 \mathrm{E}-03$ & 2.60E-03 & $2.57 \mathrm{E}-03$ & $2.54 \mathrm{E}-03$ \\
\hline & 170 & $8.96 \mathrm{E}-04$ & $8.82 \mathrm{E}-04$ & $8.62 \mathrm{E}-04$ & 8.57E-04 & $8.55 \mathrm{E}-04$ & 8.64E-04 & $8.75 \mathrm{E}-04$ \\
\hline & 171 & $3.86 \mathrm{E}-04$ & $3.88 \mathrm{E}-04$ & $3.86 \mathrm{E}-04$ & $3.83 \mathrm{E}-04$ & $3.80 \mathrm{E}-04$ & $3.79 E-04$ & $3.79 \mathrm{E}-04$ \\
\hline & 172 & $1.45 \mathrm{E}-04$ & $1.47 \mathrm{E}-04$ & $1.43 \mathrm{E}-04$ & $1.42 \mathrm{E}-04$ & $1.41 \mathrm{E}-04$ & $1.45 \mathrm{E}-04$ & $1.49 \mathrm{E}-04$ \\
\hline & 173 & $2.69 \mathrm{E}-04$ & $2.71 \mathrm{E}-04$ & $2.69 \mathrm{E}-04$ & 2.67E-04 & $2.65 \mathrm{E}-04$ & $2.64 \mathrm{E}-04$ & $2.65 \mathrm{E}-04$ \\
\hline & 174 & 7.17E-04 & $7.08 \mathrm{E}-04$ & $6.85 \mathrm{E}-04$ & $6.82 \mathrm{E}-04$ & $6.80 \mathrm{E}-04$ & $6.97 \mathrm{E}-04$ & 7.16E-04 \\
\hline & 175 & 5.36E-05 & 5.53E-05 & 4.69E-05 & 4.67E-05 & $4.72 \mathrm{E}-05$ & 5.81E-05 & 7.01E-05 \\
\hline & 176 & 1.57E-03 & $1.54 \mathrm{E}-03$ & $1.52 \mathrm{E}-03$ & $1.51 \mathrm{E}-03$ & $1.50 \mathrm{E}-03$ & $1.50 \mathrm{E}-03$ & $1.49 \mathrm{E}-03$ \\
\hline & 177 & 2.67E-04 & $2.68 \mathrm{E}-04$ & $2.65 \mathrm{E}-04$ & $2.63 \mathrm{E}-04$ & 2.61E-04 & 2.63E-04 & $2.66 \mathrm{E}-04$ \\
\hline & 178 & 4.91E-04 & $4.93 \mathrm{E}-04$ & $4.58 \mathrm{E}-04$ & $4.56 \mathrm{E}-04$ & $4.58 \mathrm{E}-04$ & $4.98 \mathrm{E}-04$ & $5.43 \mathrm{E}-04$ \\
\hline & 179 & $1.81 \mathrm{E}-03$ & $1.77 \mathrm{E}-03$ & $1.75 \mathrm{E}-03$ & $1.74 \mathrm{E}-03$ & $1.73 \mathrm{E}-03$ & $1.73 \mathrm{E}-03$ & $1.72 \mathrm{E}-03$ \\
\hline
\end{tabular}


Table 5 (continued)

\begin{tabular}{|c|c|c|c|c|c|c|c|c|}
\hline \multirow[b]{2}{*}{ i } & \multirow[b]{2}{*}{$\mathrm{j}$} & \multicolumn{7}{|c|}{ Effective collision strengths at temperatures } \\
\hline & & $650 \mathrm{eV}$ & $850 \mathrm{eV}$ & $1050 \mathrm{eV}$ & $1250 \mathrm{eV}$ & $1450 \mathrm{eV}$ & $1650 \mathrm{eV}$ & $1850 \mathrm{eV}$ \\
\hline & 180 & $4.29 \mathrm{E}-08$ & 4.19E-08 & $4.52 \mathrm{E}-08$ & $4.16 \mathrm{E}-08$ & $3.76 \mathrm{E}-08$ & $3.13 \mathrm{E}-08$ & $2.41 \mathrm{E}-08$ \\
\hline & 181 & $9.53 \mathrm{E}-08$ & $9.52 \mathrm{E}-08$ & $9.67 \mathrm{E}-08$ & $9.12 \mathrm{E}-08$ & $8.53 \mathrm{E}-08$ & 8.06E-08 & $7.51 \mathrm{E}-08$ \\
\hline & 182 & $1.66 \mathrm{E}-07$ & $1.62 \mathrm{E}-07$ & $1.63 \mathrm{E}-07$ & $1.62 \mathrm{E}-07$ & $1.61 \mathrm{E}-07$ & 1.57E-07 & $1.52 \mathrm{E}-07$ \\
\hline & 183 & $3.01 \mathrm{E}-07$ & 2.97E-07 & 3.33E-07 & 3.17E-07 & $2.99 \mathrm{E}-07$ & $2.56 \mathrm{E}-07$ & 2.06E-07 \\
\hline & 184 & $5.60 \mathrm{E}-07$ & $5.58 \mathrm{E}-07$ & $6.05 \mathrm{E}-07$ & $5.72 \mathrm{E}-07$ & $5.34 \mathrm{E}-07$ & 4.69E-07 & 3.94E-07 \\
\hline & 185 & $1.51 \mathrm{E}-07$ & 1.59E-07 & 1.27E-07 & $1.26 \mathrm{E}-07$ & $1.29 \mathrm{E}-07$ & $1.66 \mathrm{E}-07$ & $2.08 \mathrm{E}-07$ \\
\hline & 186 & 1.19E-07 & 1.17E-07 & $1.21 \mathrm{E}-07$ & 1.16E-07 & $1.09 \mathrm{E}-07$ & $1.03 \mathrm{E}-07$ & $9.55 \mathrm{E}-08$ \\
\hline & 187 & $2.49 \mathrm{E}-06$ & 2.45E-06 & 2.47E-06 & $2.38 \mathrm{E}-06$ & 2.27E-06 & $2.15 \mathrm{E}-06$ & $2.01 \mathrm{E}-06$ \\
\hline & 188 & $1.90 \mathrm{E}-07$ & 1.83E-07 & $1.72 \mathrm{E}-07$ & $1.71 \mathrm{E}-07$ & $1.70 \mathrm{E}-07$ & $1.74 \mathrm{E}-07$ & $1.80 \mathrm{E}-07$ \\
\hline & 189 & 3.07E-07 & 3.14E-07 & 2.73E-07 & $2.74 \mathrm{E}-07$ & 2.77E-07 & $3.25 \mathrm{E}-07$ & 3.78E-07 \\
\hline & 190 & $1.07 \mathrm{E}-07$ & 1.07E-07 & $1.13 \mathrm{E}-07$ & $1.08 \mathrm{E}-07$ & $1.02 \mathrm{E}-07$ & $9.51 \mathrm{E}-08$ & $8.71 E-08$ \\
\hline & 191 & $3.34 \mathrm{E}-06$ & $3.35 E-06$ & $3.94 \mathrm{E}-06$ & $3.59 \mathrm{E}-06$ & $3.21 \mathrm{E}-06$ & $2.52 \mathrm{E}-06$ & $1.72 \mathrm{E}-06$ \\
\hline & 192 & $6.34 \mathrm{E}-08$ & $6.88 \mathrm{E}-08$ & $7.72 \mathrm{E}-08$ & 7.93E-08 & 8.04E-08 & $8.29 \mathrm{E}-08$ & 8.39E-08 \\
\hline & 193 & $3.15 E-07$ & $3.14 \mathrm{E}-07$ & $3.48 \mathrm{E}-07$ & $3.24 \mathrm{E}-07$ & 2.95E-07 & $2.50 \mathrm{E}-07$ & $1.98 \mathrm{E}-07$ \\
\hline & 194 & $4.51 \mathrm{E}-08$ & 4.35E-08 & $4.21 \mathrm{E}-08$ & $4.26 \mathrm{E}-08$ & 4.34E-08 & $4.52 \mathrm{E}-08$ & $4.69 \mathrm{E}-08$ \\
\hline & 195 & $3.91 \mathrm{E}-08$ & $4.88 \mathrm{E}-08$ & $4.43 \mathrm{E}-08$ & $3.75 \mathrm{E}-08$ & $2.88 \mathrm{E}-08$ & $2.85 \mathrm{E}-08$ & $3.05 \mathrm{E}-08$ \\
\hline & 196 & $1.10 \mathrm{E}-07$ & $1.04 \mathrm{E}-07$ & $1.05 \mathrm{E}-07$ & 1.07E-07 & $1.10 \mathrm{E}-07$ & 1.10E-07 & $1.09 \mathrm{E}-07$ \\
\hline & 197 & $3.70 \mathrm{E}-08$ & $3.44 \mathrm{E}-08$ & $2.08 \mathrm{E}-08$ & $1.58 \mathrm{E}-08$ & $1.14 \mathrm{E}-08$ & $1.39 \mathrm{E}-08$ & $1.88 \mathrm{E}-08$ \\
\hline & 198 & $1.13 \mathrm{E}-07$ & $1.44 \mathrm{E}-07$ & $1.49 \mathrm{E}-07$ & $1.35 \mathrm{E}-07$ & $1.14 \mathrm{E}-07$ & 1.07E-07 & $1.04 \mathrm{E}-07$ \\
\hline & 199 & $2.83 \mathrm{E}-08$ & $2.79 \mathrm{E}-08$ & $2.48 \mathrm{E}-08$ & $2.40 \mathrm{E}-08$ & $2.66 \mathrm{E}-08$ & $3.45 \mathrm{E}-08$ & $4.02 \mathrm{E}-08$ \\
\hline & 200 & $4.17 \mathrm{E}-08$ & $5.16 \mathrm{E}-08$ & $4.23 \mathrm{E}-08$ & $3.17 \mathrm{E}-08$ & $1.96 \mathrm{E}-08$ & $2.12 \mathrm{E}-08$ & $2.65 \mathrm{E}-08$ \\
\hline & 201 & $8.74 \mathrm{E}-07$ & 8.58E-07 & 8.89E-07 & $8.78 \mathrm{E}-07$ & $8.65 \mathrm{E}-07$ & $8.24 \mathrm{E}-07$ & $7.78 \mathrm{E}-07$ \\
\hline & 202 & $4.13 \mathrm{E}-07$ & 4.74E-07 & $8.03 \mathrm{E}-07$ & $9.75 \mathrm{E}-07$ & $1.14 \mathrm{E}-06$ & 1.15E-06 & $1.11 \mathrm{E}-06$ \\
\hline & 203 & $1.57 \mathrm{E}-07$ & 1.67E-07 & $1.35 \mathrm{E}-07$ & $1.36 \mathrm{E}-07$ & $1.40 \mathrm{E}-07$ & 1.77E-07 & 2.17E-07 \\
\hline & 204 & $2.87 \mathrm{E}-07$ & $2.58 \mathrm{E}-07$ & $2.38 \mathrm{E}-07$ & $2.45 \mathrm{E}-07$ & $2.61 \mathrm{E}-07$ & 2.73E-07 & $2.84 \mathrm{E}-07$ \\
\hline & 205 & $6.09 \mathrm{E}-07$ & $5.63 \mathrm{E}-07$ & 5.09E-07 & $5.23 \mathrm{E}-07$ & $5.52 \mathrm{E}-07$ & $5.98 \mathrm{E}-07$ & $6.45 \mathrm{E}-07$ \\
\hline & 206 & $1.08 \mathrm{E}-05$ & $1.35 \mathrm{E}-05$ & $1.45 \mathrm{E}-05$ & $1.37 \mathrm{E}-05$ & $1.16 \mathrm{E}-05$ & $9.97 \mathrm{E}-06$ & $9.19 \mathrm{E}-06$ \\
\hline & 207 & $3.89 \mathrm{E}-07$ & 3.89E-07 & $3.38 \mathrm{E}-07$ & $3.45 E-07$ & $3.58 \mathrm{E}-07$ & 4.19E-07 & 4.86E-07 \\
\hline & 208 & $1.35 \mathrm{E}-06$ & $1.52 \mathrm{E}-06$ & 2.01E-06 & $2.05 \mathrm{E}-06$ & $2.03 \mathrm{E}-06$ & $1.89 \mathrm{E}-06$ & $1.70 \mathrm{E}-06$ \\
\hline & 209 & $5.20 \mathrm{E}-06$ & $5.49 \mathrm{E}-06$ & $6.42 \mathrm{E}-06$ & $6.27 \mathrm{E}-06$ & $6.01 \mathrm{E}-06$ & 5.37E-06 & $4.60 \mathrm{E}-06$ \\
\hline & 210 & $2.42 \mathrm{E}-05$ & $2.40 \mathrm{E}-05$ & 2.67E-05 & $2.56 \mathrm{E}-05$ & $2.42 \mathrm{E}-05$ & $2.10 \mathrm{E}-05$ & $1.73 \mathrm{E}-05$ \\
\hline & 211 & $5.64 \mathrm{E}-07$ & 5.97E-07 & 5.33E-07 & $5.34 \mathrm{E}-07$ & $5.38 \mathrm{E}-07$ & $6.39 \mathrm{E}-07$ & 7.47E-07 \\
\hline & 212 & $2.78 \mathrm{E}-06$ & $2.72 \mathrm{E}-06$ & $2.65 \mathrm{E}-06$ & $2.63 \mathrm{E}-06$ & 2.61E-06 & $2.64 \mathrm{E}-06$ & $2.67 \mathrm{E}-06$ \\
\hline & 213 & $7.98 \mathrm{E}-06$ & $7.78 \mathrm{E}-06$ & $7.68 \mathrm{E}-06$ & $7.62 \mathrm{E}-06$ & $7.59 E-06$ & 7.54E-06 & 7.49E-06 \\
\hline & 214 & 2.71E-05 & $2.64 \mathrm{E}-05$ & 2.63E-05 & 2.61E-05 & 2.59E-05 & $2.54 \mathrm{E}-05$ & $2.47 \mathrm{E}-05$ \\
\hline & 215 & $1.43 \mathrm{E}-06$ & $1.46 \mathrm{E}-06$ & $1.31 \mathrm{E}-06$ & $1.31 \mathrm{E}-06$ & $1.33 \mathrm{E}-06$ & $1.52 \mathrm{E}-06$ & $1.73 \mathrm{E}-06$ \\
\hline & 216 & $2.41 \mathrm{E}-06$ & $2.16 \mathrm{E}-06$ & $1.61 \mathrm{E}-06$ & $1.46 \mathrm{E}-06$ & $1.35 \mathrm{E}-06$ & $1.48 \mathrm{E}-06$ & $1.69 \mathrm{E}-06$ \\
\hline & 217 & $2.15 \mathrm{E}-06$ & $1.82 \mathrm{E}-06$ & $1.31 \mathrm{E}-06$ & $1.22 \mathrm{E}-06$ & $1.20 \mathrm{E}-06$ & $1.34 \mathrm{E}-06$ & $1.53 \mathrm{E}-06$ \\
\hline & 218 & $8.18 \mathrm{E}-06$ & $7.35 \mathrm{E}-06$ & 5.51E-06 & $4.76 \mathrm{E}-06$ & 4.11E-06 & $4.24 \mathrm{E}-06$ & 4.61E-06 \\
\hline & 219 & $1.76 \mathrm{E}-06$ & $1.59 \mathrm{E}-06$ & $1.20 \mathrm{E}-06$ & $1.10 \mathrm{E}-06$ & $1.03 \mathrm{E}-06$ & $1.15 \mathrm{E}-06$ & $1.32 \mathrm{E}-06$ \\
\hline & 220 & $1.77 \mathrm{E}-06$ & $1.72 \mathrm{E}-06$ & $1.86 \mathrm{E}-06$ & $1.86 \mathrm{E}-06$ & $1.86 \mathrm{E}-06$ & $1.73 \mathrm{E}-06$ & $1.58 \mathrm{E}-06$ \\
\hline & 221 & $7.64 \mathrm{E}-08$ & $8.10 \mathrm{E}-08$ & $6.51 \mathrm{E}-08$ & $6.58 \mathrm{E}-08$ & $6.74 \mathrm{E}-08$ & $8.56 \mathrm{E}-08$ & $1.05 \mathrm{E}-07$ \\
\hline & 222 & $2.83 \mathrm{E}-07$ & $2.72 \mathrm{E}-07$ & 2.66E-07 & $2.56 \mathrm{E}-07$ & 2.47E-07 & $2.39 \mathrm{E}-07$ & 2.30E-07 \\
\hline & 223 & $5.71 \mathrm{E}-07$ & $5.55 \mathrm{E}-07$ & 5.32E-07 & $5.16 \mathrm{E}-07$ & $5.02 \mathrm{E}-07$ & $5.05 \mathrm{E}-07$ & 5.09E-07 \\
\hline & 224 & $1.06 \mathrm{E}-05$ & $9.08 \mathrm{E}-06$ & 7.87E-06 & $8.32 \mathrm{E}-06$ & $9.13 \mathrm{E}-06$ & $9.71 \mathrm{E}-06$ & $1.04 \mathrm{E}-05$ \\
\hline & 225 & 2.61E-07 & $2.70 \mathrm{E}-07$ & $2.52 \mathrm{E}-07$ & $2.50 \mathrm{E}-07$ & 2.49E-07 & $2.75 \mathrm{E}-07$ & $3.03 E-07$ \\
\hline & 226 & $9.92 \mathrm{E}-07$ & $9.59 \mathrm{E}-07$ & $9.79 \mathrm{E}-07$ & $9.88 \mathrm{E}-07$ & $1.00 \mathrm{E}-06$ & $9.93 \mathrm{E}-07$ & $9.74 \mathrm{E}-07$ \\
\hline & 227 & $3.65 \mathrm{E}-06$ & $3.51 \mathrm{E}-06$ & $3.65 E-06$ & $3.56 \mathrm{E}-06$ & $3.47 \mathrm{E}-06$ & $3.23 \mathrm{E}-06$ & $2.95 \mathrm{E}-06$ \\
\hline & 228 & $1.64 \mathrm{E}-05$ & $1.58 \mathrm{E}-05$ & $1.66 \mathrm{E}-05$ & $1.59 \mathrm{E}-05$ & $1.52 \mathrm{E}-05$ & $1.36 \mathrm{E}-05$ & $1.18 \mathrm{E}-05$ \\
\hline & 229 & $3.41 \mathrm{E}-07$ & $3.55 \mathrm{E}-07$ & $3.12 \mathrm{E}-07$ & $3.13 E-07$ & $3.17 E-07$ & 3.77E-07 & $4.41 \mathrm{E}-07$ \\
\hline & 230 & $1.40 \mathrm{E}-06$ & $1.38 \mathrm{E}-06$ & $1.34 \mathrm{E}-06$ & $1.33 \mathrm{E}-06$ & $1.32 \mathrm{E}-06$ & $1.33 \mathrm{E}-06$ & $1.35 \mathrm{E}-06$ \\
\hline & 231 & $3.70 \mathrm{E}-06$ & $3.62 \mathrm{E}-06$ & $3.57 \mathrm{E}-06$ & $3.54 \mathrm{E}-06$ & $3.52 \mathrm{E}-06$ & $3.50 \mathrm{E}-06$ & $3.49 \mathrm{E}-06$ \\
\hline & 232 & $1.18 \mathrm{E}-05$ & $1.15 \mathrm{E}-05$ & $1.15 \mathrm{E}-05$ & $1.14 \mathrm{E}-05$ & $1.13 \mathrm{E}-05$ & 1.10E-05 & $1.08 \mathrm{E}-05$ \\
\hline & 233 & $7.24 \mathrm{E}-07$ & $7.46 \mathrm{E}-07$ & $6.55 \mathrm{E}-07$ & $6.57 \mathrm{E}-07$ & $6.66 \mathrm{E}-07$ & $7.84 \mathrm{E}-07$ & $9.13 \mathrm{E}-07$ \\
\hline & 234 & 9.67E-07 & $9.00 \mathrm{E}-07$ & $8.75 E-07$ & 8.95E-07 & $9.30 \mathrm{E}-07$ & $9.59 \mathrm{E}-07$ & $9.81 \mathrm{E}-07$ \\
\hline & 235 & $1.22 \mathrm{E}-06$ & 1.17E-06 & $1.09 \mathrm{E}-06$ & 1.07E-06 & $1.07 \mathrm{E}-06$ & $1.12 \mathrm{E}-06$ & 1.17E-06 \\
\hline & 236 & $2.30 \mathrm{E}-07$ & $2.15 \mathrm{E}-07$ & $1.63 \mathrm{E}-07$ & $1.60 \mathrm{E}-07$ & $1.59 \mathrm{E}-07$ & $1.80 \mathrm{E}-07$ & $2.10 \mathrm{E}-07$ \\
\hline & 237 & 2.07E-07 & 1.60E-07 & $9.36 \mathrm{E}-08$ & $8.11 \mathrm{E}-08$ & $6.72 \mathrm{E}-08$ & $7.11 \mathrm{E}-08$ & $1.03 \mathrm{E}-07$ \\
\hline & 238 & $3.79 E-06$ & $3.63 \mathrm{E}-06$ & $3.40 \mathrm{E}-06$ & $3.27 \mathrm{E}-06$ & $3.15 E-06$ & $3.11 \mathrm{E}-06$ & $3.09 \mathrm{E}-06$ \\
\hline & 239 & $9.84 \mathrm{E}-07$ & $9.56 \mathrm{E}-07$ & 8.17E-07 & 8.01E-07 & 7.99E-07 & $8.58 \mathrm{E}-07$ & $9.47 \mathrm{E}-07$ \\
\hline & 240 & 2.09E-07 & 2.02E-07 & $1.56 \mathrm{E}-07$ & $1.50 \mathrm{E}-07$ & $1.44 \mathrm{E}-07$ & $1.64 \mathrm{E}-07$ & $1.91 \mathrm{E}-07$ \\
\hline & 241 & $8.60 \mathrm{E}-07$ & $8.42 \mathrm{E}-07$ & 7.70E-07 & $7.65 \mathrm{E}-07$ & $7.66 \mathrm{E}-07$ & 8.33E-07 & $9.05 \mathrm{E}-07$ \\
\hline
\end{tabular}

